\title{
Selected Bibliography of Statistical Literature: Supplement, 1958-1960
}

\author{
Lola S. Deming
}

(February 8, 1963)

\begin{abstract}
This is the last of a series of bibliographies that deal with various specific subjects in the field of statistics. The preceding six bibliographies of the series gave references for the period from 1930 to 1957 . This one is intended to supplement the others by bringing them up to date through 1960. The references are arranged under the various subject titles published earlier in this series.
\end{abstract}

The purpose of this series of bibliographies is to provide a list of references in several specific subjects within the very large field of probability and mathematical statistics. The purpose of this last one in the series is to supplement the others by including references to related material published in 1958, 1959, and 1960. We felt that to carry this effort beyond 1960 would be redundant and overlapping. Within the last few years a number of bibliographic resources have become available in varying degrees of generality and specialization either as one-volume lists of references or as continuing abstract services. Although my intention is not to give a bibliography of bibliographies and abstract services, I shall mention several significant ones to show that as the statistical literature grows to a staggering amount, so also does interest grow in the construction of reference material to make the statistician aware of the existence of papers relevant to his field and to enable him to trace them.

Most noteworthy among the bibliographies is the Bibliography of Statistical Literature 1950-1958 by Maurice G. Kendall and Alison G. Doig. Professor Kendall promises that two more volumes, also covering the general field of statistical method and theory, will follow to cover the period from the 16th century up to 1950 . A number of excellent bibliographies that specialize in particular phases of statistics have also been published. Two of these cover subjects that were intended for inclusion within this present series. However, in each case the author did a more thorough search of the literature for his particular area than could have been handled here. These are: I. Richard Savage, Bibliography of Nonparametric Statistics, Harvard University Press, Cambridge, Mass., 1962; and J. Edward Jackson, Bibliography on Sequential Analysis, Jour. Amer. Stat. Assoc. Vol. 55, 561-580, Sept. 1960.

A great service to the statistician is provided by the International Statistical Institute through its two abstracting journals: International Journal of $A b$ stracts-Statistical Theory and Methods, published quarterly since 1959; and International Journal of Abstracts - Statistical Methods in Industry published three times a year since 1954. Another digest service in applied statistics is Quality Control and Applied
Statistics published by Interscience Publishers, Inc., New York.

As was explained in other sections of this series, we have limited the source material to the two large reviewing journals-Zentralblatt für Mathematik for the years 1930-1939, and Mathematical Reviews from 1940-1960. All abstracts under the general headings of "Probability" and "Mathematical Statistics" are transferred to cards. These are arranged alphabetically by author and placed in a file. This collection of abstracts is maintained on a current basis in the NBS Statistical Engineering Laboratory. The abstracts are coded into many categories of subject matter following generally, but with some minor deviations, the classification scheme of Mathematical Reviews. One abstract may be classified under several subjects; hence it may appear in more than one place in this series. References were transcribed from the abstracts by means of punched cards and titles were added to prepare the following selected subjects for publication in the NBS Journal of Research:

Correlation and Regression Theory, Vol. 64B, pp. 55-68, January-March 1960;

Time Series, Vol. 64B, pp. 69-76, January-March 1960 ;

Limit Theorems, Vol. 64B, pp. 175-192, July-September 1960;

Markov Chains and Stochastic Processes, Vol. 65B, pp. 61-93, January-March 1961;

Frequency Functions, Moments, and Graduation, Vol. 66B, pp. 15-28, January-March 1962;

Theory of Estimation and Testing of Hypotheses, Sampling Distributions, and Theory of Sample Surveys, Vol. 66B, pp. 109-151, July-September 1962 .

Most of the abstracts deal with statistical theory and methodology with applications usually appearing only when they illustrate something new or novel in theory or method. The classification scheme for the Mathematical Reviews has undergone revision from time to time and in the last several years the category "Correlation and Regression Theory" was eliminated entirely and articles on these subjects placed among the other classifications as appropriate. 
The references given here contain the following information taken directly from the abstracts:

Author: The author's surname, followed by initials only. In the case of multiple authorships, the journal reference appears with each author's name, but the title of the paper appears with the first author only. The symbol preceding the surname denotes multiple authorship.

Title: Exactly as in the reviewing journal. Titles of separately bound publications (books, reports, theses, etc.) are in italics, followed by the publisher.

Reference to literature: The name of the journal in italics and the number of the volume in bold face, are followed by initial page number.

Date of publication: The next figure, in parentheses shows the date when the article or book itself appeared.

M (for Mathematical Reviews) and Z (for Zentralblatt für Mathematik) are followed by the volume number and page number of the reviewing journal in which the abstract appears.

\section{Time Series}

Aki, K., Space and time spectra of stationary stochastic waves, with special reference to microtremors, Bull. Earthquake Res. Inst. Tokyo 35, 415 (1957).

M 19, 783

Allais, M., Test de périodicité. Généralisation du test de Schuster au cas de séries temporelles autocorréllées, C.R. Acad. Sci. Paris 244, 2469 (1957).

M 19, 475

Anderson, T. W., On asymptotic distributions of estimates of parameters of stochastic difference equations, Ann. Math. Statist. 30, 676 (1959).

M 21, 1131

Arrow, K. J., A time series analysis of interindustry demands (Contributions to Economic Analysis, XVII; North-Holland Publishing Co., Amsterdam, (1959;.

M 21, 747

Balakrishnan, A. V., On a characterization of covariances, Ann. Math. Statist. 30, 670 (1959).

M 21, 1245

Bass, J., Sur certaines classes de fonctions admettant une fonction d'autocorrélation continue, $C . R$. Acad. Sci. Paris 245, 1217 (1957). $\quad$ M 20, 468

- Bass, J., Moyennes de sommes trigonométriques et fonctions d'autocorrélation, C.R. Acad. Sci. Paris 245, 2457 (1957).

M 20, 468

- Bertrandias, J., (See J. Bass) C. R. Acad. Sci. Paris 245, 2457 (1957).

- Blackman, R. B., The measurement of power spectra from the point of view of communications engineering. I., Bell System Tech. J. 37, 185 (1958).

M 21, 323

Blackman, R. B., The measurement of power spectra from the point of view of communications engineering. II., Bell System Tech. J. 3\%, 485 (1958).

M $\boldsymbol{2 1}, 323$
- Blackman, R. B., The measurement of power spectra: From the point of view of communications engineering (Dover Publications, Inc., New York, 1959).

M 21, 323

- Bochner, S., A limit theorem for the periodogram, Ann. Math. Statist. 29, 1198 (1958). M 20, 1208

Breny, H., Recherches sur la théorie statistique des faisceaux de fibres, Mém. Soc. Roy. Sci. Liège (4) 1\%, 131 pp. (1957).

M 19, 778

Broadbent, S. R., The inspection of a Markov process, J. Roy. Statist. Soc., Ser. B 20, 111 (1958).

M 20, 1022

Campbell, L. L., On the use of Hermite expansions in noise problems, J. Soc. Indust. Appl. Math. 5, 244 (1957).

M 19, 1098

Cartwright, D. E., On estimating the mean energy of sea waves from the highest waves in a record, Proc. Roy. Soc. London, Ser. A $\mathbf{2 4 \% ,} 22$ (1958).

M 20, 231

Caughey, T. K., Response of Van der Pol's oscillator to random excitation, J. Appl. Mech. 26, 345 (1959).

M $\mathbf{2 1}, 1239$

Charnock, H., Notes on the specification of atmospheric turbulence, J. Roy. Statist. Soc., Ser. A 120, 398 (1957).

M 19, 1099

Craddock, J. M., An analysis of the slower temperature variations at Kew Observatory by means of mutually exclusive band pass filters, J. Roy Statis. Soc., Ser. A 120, 387 (1957). M 19, 1099

David, S. T., Symposium on interval estimation: Confidence intervals for parameters in Markov autoregressive schemes, J. Roy. Statist. Soc., Ser. $B$ 16, 195 (1954).

M 19, 1206

- Davies, H. M., The fitting of Markoff serial variation curves, J. Roy. Statist. Soc., Ser. B 20, 120 (1958).

M 20, 1022

Davis, R. C., Optimum vs. correlation methods in tracking random signals in background noise, Quart. Appl. Math. 15, 123 (1957). M 20, 1208

Dorogovcev, A. Ya., Statistical analysis of a stochastic difference equation, Dopovidi Akad. Nauk Ukrain. RSR 1959, 120 (1959). $\quad$ M 21,837

- Eisenpress, H. (See J. Shiskin) J. Amer. Statist. Assoc. 52, 415 (1957).

Firescu, D., Sur les fonctions d'estimation des probabilités de passage d'une chaîne de Markoff, Ann. Univ. "C. I. Parhon" Bucure ti. Ser. Sti. Nat. \%, 9 (1958).

M 20, 1209

Firescu, D., Fonctions d'estimation efficientes pour les probabilités de passage d'une chaîne de Markoff, Ann. Univ. "C. I. Parhon" Bucuresti. Ser. Sti. Nat. \%, 37 (1958). M 2147

- Fleischer, I., On the statistical treatment of stochastic processes, Ann. Math. Statis. 29, 544 (1958).

M $\mathbf{2 0}, 342$

Freiberger, W., Approximate distributions of noise power measurements, Quart. Appl. Math. 1\%, 271 (1959).

M 21, 1131 
Friedland, B., Least squares filtering and prediction of non-stationary sampled data, Information and Control 1, 297 (1958).

M 21, 73

Gayen, A. K., On auto-correlations of harmonic functions, Proc. Third Cong. on Theor. \& App. Mechanics, Bangalore, Dec. 1957, pp. 345-350. (Indian Institute of Technology, Kharagpur, (1958).

M 21, 322

Geisser, S., The distribution of the ratios of certain quadratic forms in time series, Ann. Math. Statist. 28, 724 (1957).

M 19, 897

Goodman, L. A., Simplified runs tests and likelihood ratio tests for Markoff chains, Biometrika 45, 181 (1958).

M 19, 1090

Goodman, L. A., Asymptotic distributions of "psisquared" goodness of fit criteria for $m$-th order Markov chains, Ann. Math. Statist. 99, 1123 (1958).

M 20, 1022

Goodman, L. A., On some statistical tests for $m$ - th order Markov chains, Ann. Math. Statist. 30, 154 (1959).

M 21,78

Goodman, L. A., A note on Stepanow's tests for Markov chains, Teor. Veroyatnost. i Primenen. 4, 93 (1959).

M 21, 322

Goodman, N. R., On the joint estimation of the spectra, cospectrum and quadrature spectrum of a twodimensional stationary Gaussian process, Engr. Statist. Lab., Sci. Paper 10, 168 pp. (College of Engr., N.Y. Univ., N.Y., 1957.) M 19, 1098

Grenander, U., Modern trends in time series analysis, Sankhyā 18, 149 (1957).

M 19, 1206

- Grenander, U., Toeplit: forms and their applications, Calif. Monographs in Math. Sci., 245 pp. (U. of Calif. Press, Berkeley-Los Angeles, 1958.)

M 20, 223

Grenander, U., Bandwidth and variance in estimation of the spectrum, J. Roy. Statist. Soc., Ser. B 20, 152 (1958).

M 20, 468

- Grenander, U., (See W. Freiberger) Quart. Appl. Math. 1\%, 271 (1959).

Guest, P. G., Methods for numerical calculations with the type I counter, Austral. J. Phys. 11, 143 (1958).

M 21, 447

Hannan, E. J., Testing for serial correlation in least squares regression, Biometrika 44, 57 (1957).

M 19, 333

- Hannan, E. J., (See G. S. Watson) Biometrika 43, 436 (1956).

Hannan, E. J., The variance of the mean of a stationary process, J. Roy. Statist. Soc., Ser. B 19, 282 (1957).

M 19, 1098

Hannan, E. J., The asymptotic powers of certain tests of goodness of fit for time series, J. Roy. Statist. Soc., Ser. B 20, 143 (1958). M 20,1022

Hannan, E. J., The estimation of the spectral density after trend removal, J. Roy. Statist. Soc., Ser. B 20, 323 (1958).

M 21, 78

Harybin, A. E., Analysis of errors at determining mean value of the random magnitude and its meansquare error due to finite time of observation, Avtomat. i Telemeh. 18, 304 (1957).

M 20, 231
Hoffenberg, M., (See K. J. Arrow) (Contributions to Economic Analysis, XVII; North-Holland Publishing Co., Amsterdam, 1959).

Jenkins, G. M., The spectral analysis of timeseries, J. Roy. Statist. Soc., Ser. B 19, 1 (1957).

M 19, 1097

Jowett, G. H., Statistical analysis using local properties of smoothly heteromorphic stochastic series, Biometrika 44, 454 (1957). M 19,695

- Jowett, G. H., (See H. M. Davies) J. Roy. Statist. Soc., Ser. B 20, 120 (1958).

Kamat, A.R., Contributions to the theory of statistics based on the first and second successive differences, Metron 19, 97 (1958).

M 20, 919

- Kawata, T., (See S. Bochner) Ann. Math. Statist. 29, 1198 (1958).

- Kiveliovitch, M., Les séries chronologiques et la théorie du hazard, Publ. Sci. Tech., No. 65, 129 pp. (Ministère de l'Air, Paris, 1957.) M 19, 897

Klein, L. R., The estimation of distributed lags, Econometrica 26, 553 (1958).

M 20, 1209

Kolmogorov, A. N. Statistical theory of oscillations with a continous spectrum, (Translated by Morris D. Friedman, 572 California St., Newtonville 60, Mass., 1956.) Jubilee Collection 1, 242 (Akad. Nauk Press, Moscow-Leningrad, 1947).

M 19, 190

- Kooharian, A., (See I. Fleischer) Ann. Math. Statist. 29, 544 (1958).

- Leonov, Yu. P., Estimation of parameters of the probability distribution of a random function with incomplete a priori information, Avtomat. $i$ Telemeh., 18, 985 (1957).

M $\mathbf{2 0}, 918$

- Lomnicki, Z. A., On the estimation of autocorrelation in time series, Ann. Math. Statist. 28, 140 (1957).

M 19, 590

- Lomnicki, Z. A., On estimating the spectral density function of a stochastic process, J. Roy. Statist. Soc., Ser. B 19, 13 (1957).

M 19, 1098

Longuet-Higgins, M. S., The statistical analysis of a random, moving surface, Philos. Trans. Roy. Soc. London, Ser. A $\mathbf{2 4 9 ,}(1957)$.

M 19, 328

Madansky, A., Least squares estimation in finite Markov processes, Psychometrika 24, 137 (1959).

M 21,183

Mandel, J., Fitting a straight line to certain types of cumulative data, J. Amer. Statist. Assoc. 52, 552 (1957).

M 19, 1205

Moore, P. G., Interval analysis and the logarithmic transformation, J. Roy. Statist. Soc., Ser. B 20, 187 (1958).

M 20, 596

Moran, P. A. P., Random processes in economic theory and analysis, Sankhyā 21, 99 (1959).

M 21,1131

Mourier, E., Lois de probabilité conditionnelles; existence et détermination d'un résumé exhaustif pour la discrimination entre plusieurs lois de probabilité dans des espaces de Banach, C.R. Acad. Sci. Paris 24\%, 1552 (1958). $\quad$ M $\mathbf{2 1 ,} 69$ 
- Neumann, J., (See S. Rushton) J. Roy Statist. Soc., Ser. A 120, 409 (1957).

Ogawara, M., An exact test for moving averages, Bull. Math. Statist. y, 77 (1957).

M 19, 590

Parzen, E., On consistent estimates of the spectrum of a stationary time series, Ann. Math. Statist. 28, 329 (1957).

M 19, 587

Parzen, E., On choosing an estimate of the spectral density function of a stationary time series, $A n n$. Math. Statist. 28, 921 (1957).

M 20, 341

Parzen, E., On asymptotically efficient consistent estimates of the spectral density function of a stationary time series, J. Roy. Statist. Soc., Ser. $B$ 20, 303 (1958).

M 21, 78

Phillips, A. W., The estimation of parameters in systems of stochastic differential equations, Biometrika 46, 67 (1959).

M 21, 584

Pompilj, G., Su la regressione interamente pseudolineare, Fac. Sci. Statist. Demogr. Attuar., Publ. No. 30, 18 pp. (1957).

M 21, 183

- Priestley, M. B., (See G. M. Jenkins) J. Roy. Statist. Soc., Ser. B 19, 1 (1957).

Putz, R. R., A method for the measurement of the correlation function and ordinate distributions for two time-history functions, Inst. of Eng. Res (U. of Calif., Berkeley, 39 pp., 1957.) M 19, 475

Quenouille, M. H., The analysis of multiple timeseries, Griffin's Statistical Monographs \& Courses, No. I., 105 pp. (Hafner Publishing Company, New York, 1957.)

M 19, 1205

Quenouille, M. H., The comparison of correlations in time-series, J. Roy. Statist. Soc., Ser. B 20, 158 (1958).

M 20, 469

Rosenblatt, M., A multi-dimensional prediction problem, Ark. Mat. 3, 407 (1958). M 19, 1098

Rosenblatt, M., Statistical analysis of stochastic processes with stationary residuals, The Harald Cramér volume, Probability and statistics (edited by Ulf Grenander). (Almqvist \& Wiksell, Stockholm; John Wiley \& Sons, New York; 1959.)

M 21, 1245

- Roy, G. C., (See A. K. Gayen) Proc. Third Cong. on Theor. \& App. Mechanics, Bangalore, Dec. 1957, pp. 345-350. (Indian Institute of Technology, Kharagpur, 1958.)

- Rushton, S., Some applications of time series analysis to atmospheric turbulence and oceanography, J. Roy. Statist. Soc., Ser. A 120, 409 (1957).

M 19, 1099

Sargan, J. D., The estimation of relationships with autocorrelated residuals by the use of instrumental variables, J. Roy. Statist. Soc., Ser. B 21, 91 (1959).

M 21, 1411

Saunders, K. D., A power-spectrum equation for stationary random gusts, including a sample problem, J. Aero. Sci. 25, 295 (1958). $\quad$ M 19, 1206

Scheffé, H., Experiments with mixtures, J. Roy. Statist. Soc., Ser. B 20, 344 (1958). $\quad$ M 20, 1111
Shinbrot, M., On the integral equation occurring in optimization theory with nonstationary inputs, J. Math. Phys. 36, 121 (1957).

M 19, 1206

- Shiskin, J., Seasonal adjustments by electronic computer methods, J. Amer. Statist. Assoc. 52, 415 (1957).

M 19, 897

Siddiqui, M. M., On the inversion of the sample covariance matrix in a stationary autoregressive process, Ann. Math. Statist. 29, 585 (1958).

M 20, 342

Siddiqui, M. M., Covariances of least-squares estimates when residuals are correlated, Ann. Math. Statist. 99, 1251 (1958).

M 20, 1208

- Stoller, D. S., Calculating the coefficients of certain linear predictors, Math. Tables Aids Comput. 13, 122 (1959).

M 21, 837

- Stoller, L. C., (See D. S. Stoller) Math. Tables Aids Comput. 13, 122 (1959).

Striebel, C. T., On the efficiency of estimates of trend in the Ornstein Uhlenbeck process, Ann. Math. Statist. 29, 192 (1958). $\quad$ M 20,67

Striebel, C. T., Densities for stochastic processes, Ann. Math. Statist. 30, 559 (1959). M 21, 584

Stuart, A., The efficiency of the records test for trend in normal regression, J. Roy. Statist. Soc., Ser. $B$ 19, 149 (1957).

M 19, 783

Svešnikov, A. A., Determination of the probability characteristics of three-dimensional sea-waves, Izv. Akad. Nauk SSSR Otd. Teh. Nauk. Meh. Mašinostr. 1959, 32 (1959). M 21, 584

- Szegö, G., (See U. Grenander) Calif. Monographs in Math. Sci., 245 pp. (U. of Calif. Press, BerkeleyLos Angeles, 1958.)

- Tel'ksnis, L. A., (See Yu. P. Leonov) Avtomat. $i$ Telemeh., 18, 985 (1957).

Törnqvist, L., A method for calculating changes in regression coefficients and inverse matrices corresponding to changes in the set of available data, Skand. Aktuarietidskr. 40, 219 (1957). M $\mathbf{2 0}, 814$

- Tukey, J. W., (See R. B. Blackman) Bell System Tech. J. 3\%, 185 (1958).

- Tukey, J. W., (See R. B. Blackman) Bell System Tech. J. 3\%, 485 (1958).

- Tukey, J. W., (See R. B. Blackman) (Dover Publications, Inc., New York, 1959).

Tukey, J. W., The estimation of (power) spectra and related quantities, Proc. Symp. on Numerical Approx., Madison, April 1958, pp. 389-411. (Univ. of Wis. Press, Madison, 1959.)

M $\mathbf{2 1}, 323$

- Vialar, J., (See M. Kiveliovitch) Publ. Sci. Tech., no. 65, 129 pp. (Ministère de l'Air, Paris, 1957.)

Wagner, H. M., A Monte Carlo study of estimates of simultaneous linear structural equations, Econmetrica 26, 117 (1958).

M 19, 897

Walker, A. M., The existence of Bartlett-Rajalakshman goodness of fit $G$-tests for multivariate autoregressive processes with finitely dependent residuals, Proc. Cambridge Philos. Soc. 54, 225 (1958).

M 21, 78 
Wang, S., On the estimation of regression coefficients of a random field with homogeneous residual, Acta Math. Sinica 8, 210 (1958). M 21, 584

- Watson, G. S., Serial correlation in regression analysis. II., Biometrika 43, 436 (1956). M 19, 694

Weinstein, A. S., Alternative definitions of the serial correlation coefficient in short autoregressive sequences, J. Amer. Statist. Assoc. 53, 881 (1958).

M 20, 1111

White, J. S., The limiting distribution of the serial correlation coefficient in the explosive case, $A n n$. Math. Statist. 29, 1188 (1958).

M 20, 1209

White, J. S., The limiting distribution of the serial correlation coefficient in the explosive case, II., Ann. Math. Statist. 30, 831 (1959). M 21, 1131

Whittle, P., Curve and periodogram smoothing, $J$. Roy. Statist. Soc., Ser. B 19, 38 (1957). M 19, 1098 Whittle, P., On the use of the normal approximation in the treatment of stochastic processes, J. Roy. Statist. Soc., Ser. B 19, 268 (1957). M 21,178

- Zaremba, S. K., (See Z. A. Lomnicki) Ann. Math. Statist. 28, 140 (1957).

- Zaremba, S. K., (See Z. A. Lomnicki) J. Roy. Statist. Soc., Ser. B 19, 13 (1957).

Zubrzycki, S., Remarks on random, stratifies and systematic sampling in a plane, Colloq. Math. 6, 251 (1958).

M 21, 78

\section{Limit Theorems}

Agnew, R. P., Asymptotic expansions in global central limit theorems, Ann. Math. Statist. 30, 721 (1959).

M 21, 1239

Badrikian, A., Les éléments aléatoires généralisés à valeurs dans un espace vectoriel; définitions et premiers résultats, C.R. Acad. Sci. Paris 248, 1603 (1959)

M 20, 1201

Barra, J., Du comportement de la moyenne d'un échantillon, C.R. Acad. Sci. Paris $\mathbf{2 4 4}, 2002$ (1957).

M 19, 1088

Barra, J., Lois des grands nombres pour des suites doubles de variables aléatoires, C.R. Acad. Sci. Paris 246, 2999 (1958).

$\mathrm{M} \mathbf{2 0}, 57$

Basharin, G. P., Multiplex limited number distribution of busy lines in the second cascade switchboard in a telephone system with refusals, Soviet Physics. Dokl. 121, 718 (1958).

M 21, 69

Baxter, G., A strong limit theorem for Gaussian processes, Proc. Amer. Math. Soc. \%, 522 (1956).

M 19, 890

Beck, A., Une loi forte des grands nombres pour des espaces de Banach uniformément convexes, C.R. Acad. Sci. Paris 246, 696 (1958). $\quad$ M 19, 1202

- Beck, A., A vector-valued random ergodic theorem, Proc. Amer. Math. Soc. 8, 1049 (1957). M 20, 766

Beck, A., Une loi forte des grands nombres dans des espaces de Banach uniformément convexes, Ann. Inst. H. Poincaré 16, 35 (1958).

M 21, 69

Bergström, H., On the limit theorems for convolutions of distribution functions. I. An analysis in the Weierstrass norm, J. Reine Angew. Math. 198, 121 (1957).
Bergström, H., On the limit theorems for convolutions of distribution functions. II. An analysis in the Weierstrass norm, J. Reine Angew. Math. 199, 1 (1958).

M 21, 176

Bergström, H., Konvergenzsätze über unendliche Produkte in abstrakten Halboruppen (Verallgemeinerungen der klassischen Grenzwertssätze in der Wahrscheinlichkeitsrechnung), Abh. Math. Sem. Univ. Hamburg 23, 228 (1959). M 21, 701

Billingsley, P., The invariance principle for dependent random variables, Trans. Amer. Math. Soc. 83, 250 (1956).

M 19, 891

Blackwell, D., On discrete variables whose sum is absolutely continuous, Ann. Math. Statist. 28, 520 (1957).

M 19, 467

- Blum, J. R., Central limit theorems for interchangeable processes, Canad. J. Math. 10, 222 (1958).

M $\mathbf{2 0}, 462$

Blum, J. R., A note on stochastic approximation, Proc. Amer. Math. Soc. 9, 404 (1958). M 20, 811

Blyumenfel'd, V. N., On uniqueness of the limit distribution for a system of stochastic differential equations, Dokl. Akad. Nauk SSSR (N.S.) 111, 739 (1956).

M 20, 181

- Bochner, S., A limit theorem for the periodogram, Ann. Math. Statist. 29, 1198 (1958). M 20, 1208

- Bochner, S., Strong law of large numbers for sequences of almost periodic functions, Ann. of Math. (2) 69, 718 (1959). M 21, 1211

Böge, W., Über die Charakterisierung unendlich teilbarer Wahrscheinlichkeitsverteilungen, J. Reine Angew. Math. 201, 150 (1959). M 21, 1235

Braumann, P., Some remarks about infinitely divisible probability laws, Univ. Lisboa Revista Fac. Ci. $A(2)$ 6, 265 (1957-58).

M 20, 1105

Breiman, L., A counterexample to a theorem of Kolmogorov, Ann. Math. Statist. 28, 811 (1957).

M 19, 987

Bühlmann, H., Sur l'indépendance asymptotique des variables aléatoires liées, C.R. Acad. Sci. Paris 245, 490 (1957).

M 19, 691

Bühlmann, H., Le problème "limite central" pour les variables aléatoires échangeables, C.R. Acad. Sci. Paris 246, 534 (1958).

M 19, 1088

Chernoff, H., A central limit theorem for sums of interchangeable random variables, Ann. Math. Statist. 29, 118 (1958).

M 20, 57

- Chernoff, H., (See J. R. Blum) Canad. J. Math. 10, 222 (1958).

Cogburn, R., Lois limites des termes variationnels des sommes normées, C.R. Acad. Sci. Paris 246, 3408 (1958).

M 20, 336

Cook, J. M., Rational formulae for the production of a spherically symmetric probability distribution, Math. Tables Aids Comput. 11, 81 (1957).

M 19, 466

Dobrushin, R., Central limit theorem for nonstationary Markov chains. I., Teor. Veroyatnost. $i$ Primenen. 1, 72 (1956).

M 19, 184

Dobrushin, R. L., Central limit theorem for nonstationary Markov chains. II., Teor. Veroyatnost. $i$ Primenen. 1, 365 (1956). 
Duoué, D., Sur le comportement asymptotique des suites de variable aléatoires, C.R. Acad. Sci. Paris 244, 2885 (1957).

M 19, 777

Dugué, D., Sur la convergence stochastique au sens de Cesaro et sur des différences importantes entre la convergence presque certaine et les convergences en probabilité et presque completés, Sankhya $\mathbf{1 8}$, 127 (1957).

M P0, 462

Dugué, D., Sur la convergence presque certaine des séries aléatoires, J. Math. Pures Appl. 38, 267 (1957).

M 21,975

- Erdös, P., On the central limit theorem for samples from a finite population, Magyar Tud. Akad. Mat. Kutató Int. Közl. 4, 49 (1959).

M 21,1121

Esseen, C., A moment inequality with an application to the central limit theorem, Skand. Aktuarietidskr. 39, 160 (1956).

M 19, 777

Esseen, C., On mean central limit theorems, Kungl. Tekn. Högsk. Handl, Stockholm 121, 31pp. (1958).

M 20,592

Fisz, H., A central limit theorem for stochastic processes with independent increments, Studia Math. 18, 223 (1959).

M 21, 1406

Fisz, M., Refinement of a probability limit theorem and its application to Bessel functions, Acta Math. Acad. Sci. Hungar. 6, 199 (1955).

M 19, 184

Fisz, M., A limit theorem for empirical distribution functions, Studia Math. 1\%, 71 (1958). M 20,915

Fisz, M., A limit theorem for non-decreasing random functions, Bull. Acad. Polon. Sci. Sér. Sci. Math. Astr. Phys. 6, 485 (1958).

M $\mathbb{1}, 68$

Fisz, M., On necessary and sufficient conditions for the validity of the strong law of large numbers expressed in terms of moments, Bull. Acad. Polon. Sci. Sér. Sci. Math. Astr. Phys. \%, 221 (1959).

M $\mathbf{2 1}, 975$

- Fortet, R., Les fonctions aléatoires comme éléments aléatoires dans les espaces de Banach, Studia Math. 15, 62 (1955).

M 19, 1202

Fortet, R., Lois des grands nombres pour des fonctions aléatoires à valeurs dans un espace de Banach, C.R. Acad. Sci. Paris 24\%, 1288 (1958).

M 20, 915

Fortet, R. M., Lois des grands nombres pour des éléments aléatoires genéraux, Proc. Int. Cong. Math. 3, 360 (North-Holland Pub. Co., Amsterdam, 1956).

M 20, 1017

Franckx, E., La loi forte des grands nombres des variables uniformement bornées. Critère des soussuites caracteristiques, Trabajos Estadist. 9, 111 (1958).

M 20, 1018

Geffroy, J., Stabilité presque complète des valeurs extrêmes d'un échantillon et convergence presque complète du milieu vers une limite certaine, C.R. Acad. Sci. Paris 246, 224 (1958).

M $\mathbf{2 0}, 467$

Geffroy, J., Etude de la stabilité presque certaine des valeurs extrêmes d'un échantillon et de la convergence presque certaine de son milieu, C.R. Acad. Sci. Paris $\mathbf{2 4 6 ,} 1154$ (1958). $\quad$ M $\mathbf{2 0}, 467$
Geffroy, J., Quelques extensions du théorème de M. Paul Lévy sur la convergence presque sûre des séries aléatoires à termes indépendants, C.R. Acad. Sci. Paris 249, 1180 (1959). $\quad$ M 21, 975

Ghurye, S. G., A remark on stable laws, Skand. Aktuarietidskr. 41, 68 (1958). $\quad$ M 21, 975

Gnedenko, B. V., On certain problems of the theory of probability, Ukrain. Mat. Ž. 9, 377 (1957).

M 19, 776

- Gnedenko, B. W., Grenzverteilungen von Summen unabhängiger Zufallsgrössen, German translation: (Akademie-Verlag, Berlin, 1959). $\quad$ M 21, 1121

Gumbel, E. J., Statistics of extremes (Columbia University Press, New York, 1958).

M 20, 468

Ibragimov, I. A., Some limit theorems for stochastic processes stationary in the strict sense, Dokl. Akad. Nauk SSSR 125, 711 (1959).

M 21, 726

- Izumi, S., (See S. Bochner) Ann. of Math. (2) 69, 718 (1959).

Kac, M., A class of limit theorems, Trans. Amer. Math. Soc. 84, 459 (1957).

M 19, 184

Kac, M., On rapidly mixing transformations and an application to continued fractions, Bull. Amer. Math. Soc. 64, 283 (1958), correction, 65, 67 (1959).

M 20, 593

Kahane, J. P., Sur le recouvrement d'un cercle par des arcs disposés au hasard, C.R. Acad. Sci. Paris P48, 184 (1959).

M $\mathbf{2 1}, 441$

- Kawata, T., (See S. Bochner) Ann. Math. Statist. 99, 1198 (1958).

Kemeny, J. G., A probability limit theorem requiringno moments, Proc. Amer. Math. Soc. 10, 607 (1959).

M 21, 1121

Kemperman, J. H. B., Some exact formulae for the Kolmogorov-Smirnov distributions, Nederl. Akad. Wetensch. Proc. Ser. A. $60=$ Indag. Math. 19, 535 (1957).

M 20, 461

Kendall, D. G., A note on Doeblin's central limit theorem, Proc. Amer. Math. Soc. 8, 1037 (1957).

M 19, 1088

Kesten, H., (See M. Kac) Bull. Amer. Math. Soc. 64, 283 (1958).

Kiefer, J., On the deviations of the empiric distribution function of vector chance variables, Trans. Amer. Math. Soc. 8\%, 173 (1958).

M 20, 915

- Kolmogorov, A. N., (See B. W. Gnedenko) (Akademie-Verlag, Berlin, 1959).

Kozin, F., A limit theorem for processes with stationary independent increments, Proc. Amer. Math. Soc. 8, 960 (1957).

M 19, 890

Kubilyus, I. P., Convolutions of arithmetic functions and limit theorems for sums of independent random variables, Vestnik Leningrad. Univ. 14, 30 (1959).

M 21, 907

Kuipers, L., Some remarks on asymptotic distribution functions, Arch. Math. 8,104 (1957).

M 19, 1202

Lamperti, J., Some limit theorems for stochastic processes, J. Math. Mech. '7, 433 (1958). M $\mathbf{~} \mathbf{0 0}, 811$ 
Le Cam, L., Un théorème sur la division d'un intervalle par des points pris auhasard, Publ. Inst. Statist. Univ. Paris $\mathbf{y ,} 7$ (1958).

M 21, 829

Linnik, Yu. V., General theorems of the factorization of infinitely divisible laws, Teor. Veroyatnost. i Primenen. 4, 55 (1959).

M 21, 441

Loève, Michel, A l'intérieur du problème central, Publ. Inst. Statist. Univ. Paris 6, 313 (1957).

M 20, 1201

- Lomnicki, Z. A., A further instance of the central limit theorem for dependent random variables, Math. Z. 66, 490 (1957).

M $\mathbf{2 0}, 1018$

Morimura, H., Some limit theorems concerning with the renewal numbers, Kōdai Math. Sem. Rep. 10, 47 (1958).

M 20, 593

Motoo, M., Some theorems on the sum of positive random variables, Ann. Inst. Statist. Math., Tokyo \%, 169 (1956).

M 19, 468

- Motoo, M., (See H. Watanabe) J. Math. Soc. Japan 10, 272 (1958).

Mott, J. L., The central limit theorem for a convergent non-homogeneous finite Markov chain, Proc. Roy. Soc. Edinburgh Sect. A 65, 109 (1959).

M 20, 1105

Mourier, E., L-random elements and L*-random elements in Banach spaces Proc. Third Berkeley Symp. Math. Stat. \& Prob. 2, 231. (Univ. Calif. Press, Berkeley, 1956.)

M 19, 1202

- Mourier, E., (See R. Fortet) Studia Math. 15, 62 (1955).

- Mourier, E., (See R. Fortet) C.R. Acad. Sci. Paris $\mathbf{2 4}$ \%, 1288 (1958).

Nasr, S. K., A law of large numbers for abstract random variables, Metrika 1, 89 (1958).

M 20, 915

Orey, S., A central limit theorem for $m$-dependent random variables, Duke Math. J. 25, 543 (1958).

M 20, 714

Ossoskow, G. A., Ein Grenztheorem für Folgen gleichartiger Ereignisse, Teor. Veroyatnost. i Primenen, 1, 274 (1956).

M 19, 70

Petrov, V. V., A local theorem for latticed distributions, Dokl. Akad. Nauk SSSR (N.S.) 115, 49 (1957).

M 20, 58

Petrov, V. V., Asymptotic expansions for distributions of sums of independent random variables, Teor. Veroyatnost. i Primenen. 4, 220 (1959)

M 21, 975

Petrov, V. V., On a class of limit theorems for independent random variables, Teor. Veroyatnost. $i$ Primenen. 4, 225 (1959).

M 21, 975

Postnikov, A. G., The strong law of large numbers for samples from uniformly distributed random variables, Izv. Akad. Nauk SSSR Ser. Mat. 22, 433 (1958).

M 20,335

Prékopa, A., On the independence in the limit of sums depending on the same sequence of independent random variables, Acta Math. Acad. Sci. Hungar. \%, 319 (1956).
Prékopa, A., On the compound Poisson distribution, Acta Sci. Math. Szeged 18, 23 (1957). M 19, 467

Prékopa, A., On the convergence of series of independent random variables, Publ. Math. Debrecen 4, 410 (1956).

M 19, 891

Prékopa, A., On the convergence of infinite series of independent random variables, Magyar Tud. Akad. Fiz. Oszt. Közl. 6, 191 (1956). M 20, 57

Prohorov, Yu. V., Strong stability of sums and infinitely divisible laws, Teor. Veroyatnost. i Primenen. 3, 153 (1958).

M 20,811

Prohorov, Yu. V., An extremal problem in probability theory, Teor. Veroyatnost. i Primenen. 4, 211 (1959).

M $\mathbf{2 1 ,} 829$

Prohorov, Yu. V., Some remarks on the strong law of large numbers, Teor. Veroyatnost. i Primenen. 4, 215 (1959).

M 21,829

- Rényi, A., (See A. Prékopa) Acta Math. Acad. Sci. Hungar. \%, 319 (1956).

Rényi, A., On the asymptotic distribution of the sum of a random number of independent random variables, Acta Math. Acad. Sci. Hungar. 8, 193 (1957).

M 19, 467

- Rényi, A., An inequality for uncorrelated random variables, Czechoslovak Math. J. 6 (81), 415 (1956).

M 19, 1202

Rényi, A., On a one-dimensional problem concerning random space filling, Magyar Tud. Akad. Mat. Kutató Int. Közl. 3, 109 (1958).

M 21, 577

Rényi, A., (See P. Erdös) Magyar Tud. Akad. Mat. Kutató Int. Közl. 4, 49 (1959).

Révész, P., On the limit distributions of sums of dependent random variables, Ann. Univ. Sci. Budapest. Eötvös. Sect. Math. 1, 135 (1958).

М 21, 69

Révész, P., A limit distribution theorem for sums of dependent random variables, Acta Math. Acad. Sci. Hungar. 10, 125 (1959). $\quad$ M 21, 830

Rihter, V., Lokale Grenzwertsätze für grosse Abweichungen., Teor. Veroyatnost i Primenen. 2, 214 (1957).

M 19, 1087

Rihter, V., Mehrdimensionale lokale Grenzwertsätze für grosse Abweichungen, Teor. Veroyatnost. i Primenen. 3, 107 (1958).

M $\mathbf{2 0}, 57$

Rihter, V., Local limit theorems for large deviations, Dokl. Akad. Nauk SSSR (N.S.) 115, 53 (1957).

M 20, 58

Rogers, H., A note on the law of large numbers, Proc. Amer. Math. Soc. 8, 518 (1957). M 19, 468

Rogozin, B. A., Some problems in the field of limit theorems, Teor. Veroyatnost. i Primenen. 3, 186 (1958).

М $\mathbf{2 0}, 915$

- Rosenblatt, M., (See J. R. Blum) Canad. J. Math. 10, 222 (1958).

M 20, 462

Rozanov, Yu. A., On a local limit theorem for lattice distributions, Teor. Veroyatnost. i Primenen. 2, 275 (1957).

M 19, 1088

- Rozanov, Yu. A., (See V. A. Volkonskii) Teor. Veroyatnost. i Primenen. 4, 186 (1959).

Sacks, J., Asymptotic distribution of stochastic approximation procedures, Ann. Math. Statist. 29, 373 (1958). 
Sanov, I. N., On the probability of large deviations of random magnitudes, Mat. Sb. N.S. 42 (84), 11 (1957).

M 19, 466

Schnell, E., On a conditional limiting distribution theorem, Magyar Tud. Akad. Mat. Kutató Int. Közl.4, 3 (1959).

M 21, 1406

- Schwartz, J. T., (See A. Beck) Proc. Amer. Math. Soc. 8, 1049 (1957).

M 20, 766

Shapiro, J. M., Sums of independent truncated random variables, Ann. Math. Statist. 28, 754 (1957).

M 19, 1087

Shapiro, J. M., Sums of powers of independent random variables, Ann. Math. Statist. 29, 515 (1958).

M 20, 592

Siraždinov, S. H., On exact estimation in a local limit theorem, Teor. Veroyatnost. i Primenen. 4, 229 (1959).

M 21, 829

Skorohod, A. V., Limit theorems for stochastic processes with independent increments, Teor. Veroyatnost. $i$ Primenen. $\quad \mathbf{2 ,} 145$ (1957). $\quad$ M 20,224 Spitzer, F., Some probability limit theorems, Bull. Amer. Math. Soc. 65, 117 (1959). M 21, 829

Stanojević, C. V., On a theorem of K. L. Chung., Bull. Soc. Math. Phys. Serbie 8, 59 (1956).

M 19, 184

Statulyavičus, V. A., On a local limit theorem for inhomogeneous Markoff chains, Dokl. Akad. Nauk SSSR (N.S.) 10\%, 516 (1956).

M 19, 71

Statulyavičus, V. A., A local limit theorem for nonhomogeneous Markoff chains with an enumerable number of possible states, Dokl. Akad. Nauk SSSR (N.S.) 115, 872 (1957).

M 20, 462

Steck, G. P., Limit theorems for conditional distributions, Univ. California Publ. Statist. 2, 237 (1957).

M 19, 986

Studnev, Yu. P., On the behavior of the remainder term under the conditions of the theorem of Lyapunov., Užgorod. Gos. Univ. Naučn. Zap. 18, 183 (1957).

M $\mathbf{2 0}, 591$

Sukhatme, B. V., Joint asymptotic distribution of the median and a U statistic, J. Roy. Statist. Soc., Ser. B 19, 144 (1957).

M 19, 777

Takács, L., On certain sojourn time problems in the theory of stochastic processes, Acta Math. Acad. Sci. Hungar. 8, 169 (1957).

M 19, 467

Takács, L., On limiting distributions concerning "a sojourn time problem, Acta Math. Acad. Sci. Hungar. 8, 279 (1957).

M 20, 464

Teicher, H., On the convergence of projected distributions, Ann. Inst. Statist. Math., Tokyo 9, 79 (1958).

M 19, 1088

Teicher, H., (See H. Chernoff) Ann. Math. Statist. 29, 118 (1958).

- Teicher, H., (See J. R. Blum) Canad. J. Math. 10, 222 (1958).

Trotter, H. F., An elementary proof of the central limit theorem, Arch. Math. 10, 226 (1959).

M 21, 1406

Tucker, H. G., A generalization of the GlivenkoCantelli theorem, Ann. Math. Statist. 30, 828 (1959).

M $\mathbf{2 1}, 1234$
Udagawa, M., On some limit theorems for the sums of identically distributed independent random variables, Kōdai Math. Sem. Rep. 8, 85 (1956).

M 19, 891

Ueno, T., Some limit theorems for temporally discrete Markov processes, J. Fac. Sci. Univ. Tokyo. Sect. I. \%, 449 (1957).

M 19, 891

Urbanik, K., A limit theorem for a posteriori distributions, Bull Acad. Polon. Sci. Cl. III, 5, 237 (1957).

M 19, 691

Urbanik, K., On the limiting probability distribution on a compact topological group, Fund. Math. 44, 253 (1957).

M 19, 1180

Volkonskiǐ, V. A., Some limit theorems for random functions. I., Teor. Veroyatnost. i Primenen. 4, 186 (1959).

M 21, 829

Wallace, D. L., Asymptotic approximations to distributions, Ann. Math. Statist. 29, 635 (1958).

M 20, 592

Wang, S., On the limit of the common distribution of terms of variational series, Acta Math. Sinica 6, 389 (1956).

M 20, 593

Watanabe, H., On the Poisson distribution, $J$. Math. Soc. Japan 8, 127 (1956). M 19, 70

- Watanabe, H., Ergodic property of recurrent diffusion processes, J. Math. Soc. Japan 10, 272 (1958).

M 21,73

Weiss, I., Limiting distributions in some occupancy problems, Ann. Math. Statist. 29, 878 (1958).

M 20, 810

Weiss, M., On the law of the iterated logarithm, $J$. Math. Mech. 8, 121 (1959).

M 21, 315

Weiss, M., On the law of the iterated logarithm for uniformly bounded orthonormal systems, Trans. Amer. Math. Soc. 92, 531 (1959). $\quad$ M 21,1087

Weiss, M., On Hardy-Littlewood series, Trans. Amer. Math. Soc. 91, 470 (1959).

M 21, 1371

Whittle, P., Sur la distribution du maximum d'un polynome trigonométrique à coefficients aléatoires, Colloques Internationaux du Centre National de la Recherche Scientifique, Paris IXXXXVII, pp. 173184 (1959).

M 21, 829

Wigner, E. P., On the distribution of the roots of certain symmetric matrices, Ann. of Math. (2) $6 \%$, 325 (1958).

M 20, 335

Wolfowitz, J., (See J. Kiefer) Trans. Amer. Math. Soc. 8\%, 173 (1958).

Zaremba, S. K., Note on the central limit theorem, Math. Z. 69, 295 (1958). M 20, 914

Zaremba, S. K., (See Z. A. Lomnicki) Math. Z. 66, 490 (1957).

Zaremba, S. K., On necessary conditions for the central limit theorem, Math. Z. Fo, 281 (1958).

M 21, 1121

Zergényi, E., (See A. Rényi) Czechoslovak Math. J. 6 (81), 415 (1956).

Zlatev, Iv., Über die asymptotische Verteilung sich in einem Rechteck bewegender Punkte., C.R. Acad. Bulgare Sci. 12, 109 (1959). 
Markov Chains and Stochastic Processes

\section{A. Stochastic Processes-General Theory}

Akaike, H., On a zero-one process and some of its applications, Ann. Inst. Statist. Math., Tokyo 8, $87^{(1956) \text {. }}$

M 19, 327

Andreev, N. I., General condition of extremum of given function of mean-square error and of mathematical expectation square of error of dynamic system, Avtomat. $i$ Telemeh. 20, 833 (1959).

M 21, 1166

Bartoszyński, R., Some remarks on the convergence of stochastic processes, Studia Math. 1\%, 313 (1958).

M 21, 725

Bell, C. B., On the structure of stochastic independence, Illinois J. Math. 2, 415 (1958). M P1, 19

Bellman, R., On the representation of the solution of a class of stochastic differential equations, Proc. Amer. Math. Soc. 9, 326 (1958).

M 20, 462

Béthoux, P., Filtrage d'une fonction aléatoire dont la moyenne est une fonction linéaire, C.R. Acad. Sci. Paris 248, 3685 (1959).

M 21, 830

- Blum, J. R., On the structure of infinitely divisible distributions, Pacific J. Math. 9, 1 (1959).

M 21, 828

Blumenthal, R. M., Some relationships involving subordination, Proc. Amer. Math. Soc. 10, 502 (1959).

M 21, 830

- Blumenthal, R. M., The asymptotic distribution of the eigenvalues for a class of Markov operators, Pacific J. Math. 9, 399 (1959).

M 21, 1122

Breny, H., Recherches sur la théorie statistique des faisceaux de fibres, Mèm. Soc. Roy. Sci. Liège (4) 1\%, No. 2, 131 pp. (1957).

M 19, 778

Burkholder, D. L., On a class of stochastic approximation processes, Ann. Math. Statist. 2\%, 1044 (1956).

M 19, 71

Cameron, R. H., Inversion formulae for characteristic functionals of stochastic processes, $A n n$. of Math. (2) 69, 15 (1959).

M 21, 1406

Cartwright, D. E., The prediction of a random function, given simultaneous values of its first. few derivatives, J. Math. Phys. 3\%, 229 (1958).

M 20, 1018

Castoldi, L., Apparenze conduttive e propagative in processi markoviani speciali. Processi conservativi e processi livellativi, Rend. Sem. Fac. Sci. Univ. Cagliari 26, 156 (1956) M 19, 468

Cheng, S., Harmonizable stochastic process and linear translatable stochastic functional equations, Acta Math. Sinica 8, 281 (1958). M 20, 1202

Chernikov, N. A., A generalized problem on the stochastic motion of a particle, Dokl. Akad. Nauk SSSR (N.S.) 112, 1030 (1957).

M 19, 1138

Chover, J., Conditions on the realization of prediction by measures, Duke Math. J. 25, 305 (1958).

M $\mathbf{2 0}, 462$

Ciucu, G., Propriétés ergodiques de certaines chaînes à liaisons complètes, Acad. R. P. Romin̂e. Stud. Cerc. Mat. 8, 413 (1957).

M 20,336
Ciucu, G., Propriétés asymptotiques des chaînes à liaisons complètes, Atti Accad. Naz. Lincei Rend. Cl. Sci. Fis. Mat. Nat. (8) 23, 11 (1957).

M 20, 594

Cramér, H., Remarques sur le problème de prédiction pour certaines classes de processus stochastiques, Colloques Internationaux du Centre National de la Recherche Scientifique, Paris LXXXVII, pp. 103-112 (1959).

M 21, 1236

- Derman, C., On Dvoretzky's stochastic approximation theorem, Ann. Math. Statist. 30, 601 (1959).

M 21,1235

Dinculeanu, N., Remarques sur les mesures dans les espaces produits, Colloq. Math. 5, 51 (1957).

M 19, 1167

Dobrušin, R. L., The continuity condition for sample martingale functions, Teor. Veroyatnost. i Primenen 3, 97 (1958).

M 20, 224

Donsker, M. D., (See R. H. Cameron) Ann. of Math. (2) 69, 15 (1959).

Doob, J. L., Stochastic processes (Izdat. Inostr. Lit., Moscow, 1956).

M 19, 71

Dupač, V., Notes on stochastic approximation methods, Czechoslovak Math. J. 8, (83), 139 (1958).

M 20, 462

Elliott, J., Absorbing barrier processes connected with the symmetric stable densities, Illinois $J$. Math. 3, 200 (1959).

M $\mathbf{2 1}, 293$

Feldman, J., Equivalence and perpendicularity of Gaussian processes, Pacific J. Math., 8, 699 (1958). M $\mathbf{2 1 , 2 9 5}$

Feldman, J., Correction to "Equivalence and perpendicularity of Gaussian processes", Pacific J. Math. 9, 1295 (1959).

M 21, 1407

Feller, W., On boundaries defined by stochastic matrices. Applied probability, Proc. Symposia in Applied Math. VII, pp. 35-40 (McGraw-Hill Book Co, New York, 1957). M 19, 988

Fisz, H., A central limit theroem for stochastic processes with independent increments, Studia Math. 18, 223 (1959).

M $\mathbf{2 1}, 1406$

Fisz, M., (See Yu. V. Prohorov) Teor. Veroyatnost. i Primenen. 2, 475 (1957).

Fisz, M., A central limit theorem for some stochastic processes, Bull. Acad. Polon. Sci. Ser. Sci. Math. Astr. Phys. 6, 437 (1958). M 20, 463

- Fleischer, I., On the statistical treatment of stochastic processes, Ann. Math. Statist. 29, 544 (1958). M $\mathbf{2 0}, 342$

Fortet, R. M., Les fonctions aléatoires comme éléments aléatoires dans un espace de Banach, Proc. Internat'l. Congress of Math., Amsterdam III, pp. 356-359 (North-Holland Publishing Co., Amsterdam, 1956). M 19, 1089

- Frenkiel, F., (See J. Kampé de Fériet) C.R. Acad. Sci. Paris 249, 348 (1959).

Freund, J. E., Some results on recurrent events, Amer. Math. Monthly 64, 718 (1957). M 20, 916

Getoor, R. K., Markov operators and their associated semi-groups, Pacific J. Math. 9, 449 (1959). M 21,1121 
- Getoor, R. K., (See R. M. Blumenthal) Pacific J. Math. 9, 399 (1959).

Gnedenko, B. V., On certain problems of the theory of probability, Ukrain. Mat. Z. 9, 377 (1957).

M 19, 776

- Grenander, U., Toeplitz forms and their applications, Calif. Monographs in Math. Sci., $245 \mathrm{pp}$. (Univ. of Calif. Press, Berkeley-Los Angeles, 1958.)

M 20,223

Hájek, J., On a property of normal distribution of any stochastic process, Czechoslovak Math. J. 8 (83), 610 (1958).

M 21, 578

Helms, L. L., Mean convergence of martingales, Trans. Amer. Math. soc. 8\%, 439 (1958).

M $\mathbf{2 0}, 224$

Husu, A. P., On some functionals on random fields, Vestnik Leningrad Univ. 12, 37 (1957). M 19,468

- Jenkins, G. M., The spectral analysis of timeseries, J. Roy. Statist. Soc., Ser. B 19, 1 (1957).

M 19, 1097

Kac, M., Distribution of eigenvalues of certain integral operators, Michigan Math. J. 3, 141 (1955).

M 19, 70

Kac, M., Probability and related topics in physical sciences, Proc. Summer Seminar, Boulder, Colo., 1957, Vol. I. (Interscience Publishers, New York, 1959.)

M 21, 314

Kac, M., Some remarks on stable processes with independent increments, The Harald Cramér volume, pp. 130-138 (John Wiley Sons, New York, 1959).

M 21, 1236

- Kampé de Fériet, J., Estimation de la corrélation d'une fonction aléatoire non stationnaire, C.R. Acad. Sci. Paris 249, 348 (1959).

M 21, 1122

Kellerhals, G., Randwertprobleme bedingter Wahrscheinlichkeiten, Math. Z. \%1, 1 (1959).

M 21,1235

Kendall, D. G., Some further pathological examples in the theory of denumerable Markov processes, Quart. J. Math. Oxford Ser. (2) y, 39 (1956).

M 19, 586

- Kendall, D. G., Some pathological Markov processes with a denumerable infinity of states and the associated semigroups of operators on $l$, Proc. Int. Cong. Math. 3, pp. 377-415 (North-Holland Pub. Co., Amsterdam, 1956).

M 19, 586

van Klinken, J., The theory of random processes and actuarial statistics. Dependent and independent probabilities, Mitt. Verein. Schweiz. Versich.-Math. 59, 139 (1959).

M 21, 1308

Kochen, M., Circle networks of probabilistic transducers, Information and Control $\mathbf{2 ,} 168$ (1959).

M $\mathbf{2 1}, 891$

-Kooharian, A., (See I. Fleischer) Ann. Math. Statist. 29, 544 (1958).

Korezlioglu, H., Sur la prévision et le filtrage linéaires des processus discrets du second ordre, C.R. Acad. Sci. Paris 248, 356 (1959).

M 21, 578

Korezlioglu, H., Extension du théorème de Pinsker, C.R. Acad. Sci. Paris 248, 523 (1959). M 21, 578
Krickeberg, K., Convergence of martingales with a directed index set, Trans. Amer. Math. Soc. 83, 313 (1956).

M 19, 947

Krickeberg, K., Stochastische Konvergenz von Semi-martingalen, Math. Z. 66, 470 (1957).

M 19, 948

Krickeberg, K., Stochastische Derivierte, Math. Nachr. 18, 203 (1958).

M 21, 20

Krickeberg, K., Semi-martingales á base filtrante décroissante, Colloques Internationaux du Centre National de la Recherche Scientifique, Paris, LXXXVII, pp. 133-138, (1959). M $\mathbf{2 1 , 8 3 0}$

Lamperti, J., Stationary measures for certain stochastic processes, Pacific J. Math. 8, 127 (1958).

M 20,593

LeCam, L., Convergence in distribution of stochastic processes, Univ. Calif. Publ. Statist. 2, 207 (1957).

M 19, 128

Legoupil, J., Sur la dépendance de probabilité entre une variable aléatoire et une fonction aléatoire, C.R. Acad. Sci. Paris 249, 1444 (1959). M 21, 1236

Lévy, P., Sur quelques problèmes de la théorie des liaisons stochastiques, C.R. Acad. Sci. Paris $\mathbf{2 4 4 ,}$ 1313 (1957).

M 19, 185

Lévy, P., Sur une classe de courbes de l'espace de Hilbert et sur une équation intégrale non linéaire, Ann. Sci. Ecole Norm. Sup. (3) 73, 121 (1956).

M 20, 463

Lévy, P., Fonctions aléatoires à corrélation linéaire, Illinois J. Math. 1, 217 (1957). $\quad$ M 20, 1202

Lévy, P., Sur quelques classes de fonctions aléatoires, J. Math. Pures Appl. (9) 38, 1 (1959). M 21, 976

Lloyd, S. P., A sampling theorem for stationary (wide sense) stochastic processes, Trans. Amer. Math. Soc. 92, 1 (1959). $\quad$ M 21, 1122

Lomnicki, Z. A., On estimating the spectral density function of a stochastic process, J. Roy. Statist. Soc., Ser. B 19, 13 (1957). $\quad$ M 19, 1098

Masani, P., Sur la fonction génératrice d'un processus stochastique vectoriel, C.R. Acad. Sci. Paris $\mathbf{2 4 9 ,}$ 360 (1959).

M 21, 1122

Mercer, A., Some simple duration-dependent stochastic processes, J. Roy. Statist. Soc., Ser. B $\mathbf{2 1}$, 144 (1959).

M 21, 1235

Mihoc, G., Une application de la théorie des réserves mathématiques à l'étude des processus stochastiques, An. Univ. "C. I. Parhon" București. Ser. Sti. Nat. 5, no. 12, 13 (1956). $\quad$ M $\mathbf{2 0}, 593$

Minlos, R. A., Continuation of a generalized random process to a completely additive measure, Dokl. Akad. Nauk SSSR (N.S.) 119, 439 (1958).

M $\mathbf{2 0}, 915$

Minlos, R. A., Generalized random processes and their extension in measure, Trudy Moskov. Mat. Obšč. 8, 497 (1959).

M $\mathbf{2 1}, 1406$

Mourier, E., Lois de probabilité conditionnelles: existence et détermination d'un résumé exhaustif pour la discrimination entre plusieurs lois de probabilité dans des espaces de Banach, C.R. Acad. Sci. Paris 24\%, 1552 (1958). $\quad$ M 21, 69 
Nasr, S. K., Determination of the Mourier mean of random variables situated in some Banach spaces, Proc. Math. Phys. Soc. Egypt 5, no. 4, 79 (1956).

M 19, 1202

Nelson, E., Regular probability measures on function space, Ann. of Math. (2) 69, 630 (1959).

M 21, 830

Nisio, M., On a new definition of stochastic integral by random Riemann sum, Mem. Coll. Sci. Univ. Kyoto. Ser A. Math. 31, 25 (1958). $\quad$ M $\mathbf{2 1 , 6 9}$

Parzen, E., Conditions that a stochastic process be ergodic, Ann. Math. Statist. 29, 299 (1958).

M 20, 336

Pérez, A., Notions généralisées d'incertitude, d'entropie et d'information du point de vue de la théorie de martingales, Trans. First Prague Conference, Nov. 28-30, 1956, pp. 183-208. (Publishing House. Czech. Acad. Sci., Prague, 1957.)

M 20, 1040

Pérez, A., Sur la théorie de l'information dans le cas d'un alphabet abstrait, Trans. First Prague Conference, Nov. 28-30, 1956, pp. 209-243. (Publishing House Czech. Acad. Sci., Prague, 1957.)

M 20, 1041

Pérez, A., Sur la convergence des incertitudes, entropies et informations echantillon (sample) vers leurs valeurs vraies, Trans. First Prague Conference, Nov. 28-30, 1956, pp. 245-252. (Publishing House Czech. Acad. Sci., Prague, 1957.)

M 20, 1041

Phillips, A. W., The estimation of parameters in systems of stochastic differential equations, Biometrika 46, no. 1/2, 67 (1959).

M 21, 584

Pollaczek, F., Problèmes stochastiques posés par le phénomène de formation d'une queue d'attente à un guichet et par des phénomènes apparentés, Mémor. Sci. Math., No. 136 (Gauthier-Villars, Paris, 1957).

M 19, 987

Praporgescu, N., Sur les probabilités en chaîne, Acad. R. P. Romîne. Stud. Cerc. Mat. 9, 439 (1958).

M 21, 441

Prékopa, A., On the compound Poisson distribution, Acta Sci. Math. Szeged 18, 23 (1957). M 19, 467

Prékopa, A., On the independence in the limit of sums depending on the same sequence of independent random variables, Acta Math. Acad. Sci. Hungar. \%, 319 (1956). M 19, 467

Prékopa, A., On Poisson and composed Poisson stochastic set functions, Studia Math. 16, 142 (1957).

M 20, 715

Prékopa, A., On stochastic set functions. I., Acta Math. Acad. Sci. Hungar. \%, 215 (1956).

M 20,715

Prékopa, A., Stochastic set functions. I., Magyar Tud. Akad. Mat. Fiz. Oszt. Közl. 6, 289 (1956).

M 20, 715

Prékopa, A., On stochastic set functions. II., III., Acta Math. Acad. Sci. Hungar. 8, 337 (1957).

M 20,716

Prékopa, A., Stochastic set functions. II. A new stochastic integral, Magyar Tud. Akad. Mat. Fiz. Ozst. Közl. \%, 339 (1957).
- Priestley, M. B., (See G. M. Jenkins) J. Roy. Statist. Soc., Ser. B. 19, 1 (1957).

- Prohorov, Yu. V., A characterisation of normal distributions in Hilbert space, Teor. Veroyatnost. $i$ Primenen. 2, 475 (1957).

M 20, 58

Prouza, L., Bemerkung zur linearen Prediktion mittels eines lernenden Filters, Trans. First Prague Conference, Nov. 28-30, 1956, pp. 37-41. (Publishing House Czech. Acad. Sci., Prague, 1957.)

M 20, 811

Régnier, A., Sur les perturbations aléatoires des systèmes d'équations différentielles, Colloques Internat'l. du Centre National de la Recherche Scientifique, Paris LXXXVII, pp. 149-155 (1959). M 21,1235

- Rényi, A., (See A. Prékopa) Acta Math. Acad. Sci. Hungar. \%, 319 (1956).

- Rényi, A., On the asymptotic distribution of the sum of a random number of independent random variables, Acta Math. Acad. Sci. Hungar. 8, 193 (1957).

M 19, 467

- Reuter, G. E. H., (See D. G. Kendall) Proc. Int. Cong. Math. 3, pp. 377-415 (North-Holland Pub. Co., Amsterdam, 1956).

- Rosenblatt, M., (See J. R. Blum) Pacific J. Math. 9, 1 (1959).

Rozanov, Yu. A., On the linear interpolability of stationary processes in discrete time, Dokl. Akad. Nauk SSSR (N.S.) 116, 923 (1957). M 20,336

Rozenblat-Rot, M., Entropy of stochastic processes, Dokl. Akad. Nauk SSSR (N.S.) 112, 16 (1957).

M 19, 71

- Sacks, J., (See C. Derman) Ann. Math. Statist. 30, 601 (1959).

Sales, Vallés, F., On periodic random functions, Collect. Math. 9, 105 (1957). $\quad$ M 19, 1202 Sentis, P., Les répartitions en classes et quelquesunes de leurs applications, Publ. Inst. Statist. Univ. Paris \%, 15 (1958).

M $\mathbf{2 1}, 315$

Sevast'yanov, B. A., Limit theorems for branching stochastic processes of special form, Teor. Veroyatn.ost. i Primenen. 2, 339 (1957). $\quad$ M 19, 1088

Shinbrot, M., On a method for optimization of timevarying linear systems with non-stationary inputs, NACA Tech. Note no. 3991, 39 pp. (1956).

M 19, 71

Siegel, A., Stochastic basis of the Eulerian variational principle in linear dissipative processes, Phys. Rev. (2) 106, 609 (1957).

M 19, 79

Skorohod, A. V., Limit theorems for stochastic processes with independent increments, Teor. Veroyatnost. i Primenen. 2, 145 (1957). M $\mathbf{2 0 ,} 224$

Skorohod, A. V., On the differentiability of measures which correspond to stochastic processes. I. Processes with independent increments, Teor. Veroyatnost. i Primenen. 2, 417 (1957).

M $\mathbf{2 0}, 594$

Striebel, C. T., Densities for stochastic processes, Ann. Math. Statist. 30, 559 (1959). M 21, 584 Szegö, G., (See U. Grenander) Calif. Monographs in Math. Sci., 245 pp. (Univ. of Calif. Press, BerkeleyLos Angeles, 1958). 
Takács, L., On certain sojourn time problems in the theory of stochastic processes, Acta Math. Acad. Sci. Hungar. 8, 169 (1957).

M 19, 467

Takács, L., On some probability problems concerning the theory of counters, Acta Math. Acad. Sci. Hungar. 8, 127 (1957).

M 19, 777

Theodorescu, R., Stochastische kontinuierliche Prozesse mit volkommenen Verbindungen, Math. Nachr. 16, 79 (1957).

M 19, 891

Theodorescu, R., Un théorème ergodique pour les processus stochastiques continus de multiplicité p, An. Univ. "C. I. Parhon" Bucuresti. Ser. Sti. Nat. 5, no. 10, 23 (1956).

M 20, 593

Theodorescu, R., Sur un équation rencontrée dans la théorie des processus stochastiques, An. Univ. "C. I. Parhon" Bucuresti. Ser. Sti. Nat. 5, no. 11, 39 (1956).

M $\mathbf{2 0}, 593$

Tsurumi, S., On the recurrence theorems in ergodic theory, Proc. Japan Acad. 34, 208 (1958).

M 21, 725

Urbanik, K., Stochastic processes whose sample functions are distributions, Teor. Veroyatnost. $i$ Primenen. 1, 146 (1956).

M 19, 326

Urbanik, K., Generalized stochastic processes, Studia Math. 16, 268 (1958).

M $\mathbf{2 0}, 715$

Urbanik, K., The values at the fixed moment of generalized stochastic processes, Acta Math. Sinica 8, 146 (1958).

M 21,176

Urbanik, K., The values at the fixed moment of generalized stochastic processes, Sci. Sinica \%, 1 (1958).

M 21, 176

Urbanik, K., Effective processes in the sense of H. Steinhaus, Studia Math. 1\%, 335 (1958).

M 21, 578

Urbanik, K., Local characteristics of generalized stochastic processes, Studia Math. 1\%, 199 (1958).

M 21, 1124

Varadarayan, V., An existence theorem for probability spaces, Uspehi Mat. Nauk 13, 167 (1958).

M 20, 1105

Vinokurov, V. G., Conditions for the regularity of stochastic processes, Dokl. Akad. Nauk SSSR (N. S.) 113, 959 (1957).

M $\mathbf{2 0}, 224$

Wang, S., A note on generalized stochastic processes, Sci. Record (N.S.) 2, 15 (1958).

M 21, 177

Whittle, P., Curve and periodogram smoothing, J. Roy. Statist. Scc., Ser. B 19, 38 (1957).

M 19, 1098

Whittle, P., Continuous generalizations of Tchebichev's inequality. Teor. Veroyatnost. i Primenen. 3, 386 (1958).

M $\mathbf{2 1}, 1236$

Wiener, N., Nonlinear problems in random theory, Technology Press Research Monographs. (Mass. Institute of Technology and John Wiley \& Sons, Inc., New York, 1958.)

M 20, 1201

Winkelbauer, K., On the theory of generalized random processes, Crechoslovak Math. J. 6 (81), 517 (1956).

M 19, 778

Wolfowitz, J., On stochastic approximation methods, Ann. Math. Statist. 2\%, 1151 (1956).
Yaglom, A. M., Correlation theory of processes with random stationary $n$th increments, Amer. Math. Soc. Transl. (2) 8, 87 (1958).

M 19, 987

Yaglom, A. M., Certain types of random fields in $n$-dimensional space similar to stationary stochastic processes, Teor. Veroyatnost. i Primenen. 2, 292 (1957).

M $\mathbf{2 0}, 224$

Yaglom, A. M., Extrapolation, interpolation and filtering of stationary random processes with rational spectral density, Advancement in Math. 2, 161 (1956).

M 20, 715

Yaglom, A. M., Correlation theory of processes with stationary $n$th increments, Advancement in Math. 2, 202 (1956)

M 20, 715

Yaglom, A. M., Introduction to the theory of stationary random functions, Advancement in Math. 2, 3 (1956).

M 20, 715

Zaremba, S. K., (See Z. A. Lomnicki) J. Roy Statist. Soc., Ser. B 19, 13 (1957).

Zítek, F., Equations différentielles stochastiques, Czechoslovak Math. J. 8 (83), 465 (1958).

M 21, 441

Zítek, F., Sur l'intégrabilité d'une équation différentielle stochastique, Czechoslovak Math. J. 8 (83), 473 (1958).

M 21, 441

Zubrzycki, S., Remarks on random, stratified and systematic sampling in a plane, Colloq. Math. 6, 251 (1958).

M $\mathbf{2 1}, 78$

\section{B. Markov Processes}

- Aczél, J., Remarques algébriques sur la solution donnée par M. Frechet a l'equation de Kolomgoroff. II, Publ. Math. Debrecen 5, 60 (1957).

M 19, 891

Akaike, H., On optimum character of von Neumann's Monte Carlo model, Ann. Inst. Statist. Math., Tokyo \%, 183 (1956). $\quad$ M 19, 1096

Ambarcumyan, G. A., Moments of distribution of a Markov process, Akad. Nauk Armyan. SSR. Izv. Fiz. Mat. Estest. Tehn. Nauki 9, no 5, 25 (1956).

M 19, 989

Ambarcumyan, G. A., Entropy of Markov chains, Izv. Akad. Nauk Armyan. SSR. Ser. Fiz.-Mat. Nauk 11, no. 2, 31 (1958). $\quad$ M 21, 316

- Austin, D. G., On continuity of transition functions, Duke Math. J. 25, 533 (1958). $\quad$ M 20, 463

Austin, D. G., A new proof of the strong Markov theorem of Chung., Proc. Nat. Acad. Sci. U.S.A. 44, 575 (1958).

M 20, 1205

Austin, D. G., Note on differentiating Markoff transition functions with stable terminal states, Duke Math. J. 25, 625 (1958).

M 21, 71

Austin, D. G., The generalized backward Kolmogorov equation in abstract space, Illinois $J$. Math. 3, 532 (1959).

M 21, 1237

Baxter, G., An operator identity, Pacific J. Math. 8, 649 (1958).

M $\mathbf{2 1}, 441$

- Bharucha-Reid, A. T., Generating functions and the semigroup theory of branching Markov processes, Proc. Nat. Acad. Sci. U.S.A. 44, 1057 (1958). 
Bharucha-Reid, A. T., Ergodic projections for semigroups of periodic operators, Studia Math. 1\%, 189 (1958).

M 20, 1096

Blackwell, D., Another countable Markov process with only instantaneous states, Ann. Math. Statist. 29, 313 (1958).

M 20, 58

- Blackwell, D., On the identifiability problem for functions of finite Markov chains, Ann. Math. Statist. 28, 1011 (1957).

M 20, 916

Blackwell, D., The entropy of functions of finitestate Markov chains, Trans. First Prague Conference, Nov. 28-30, 1956, pp. 13-20. (Publishing House Czech. Acad. Sci., Prague, 1957.)

M 20, 1105

Blumenthal, R. M., An extended Markov property, Trans. Amer. Math. Soc. 85, 52 (1957). M 19, 468

- Blumenthal, R. M., (See D. G. Austin) Duke Math. J. 25, 533 (1958).

- Blumenthal, R. M., The asymptotic distribution of the eigenvalues for a class of Markov operators, Pacific J. Math. 9, 399 (1959).

M $\mathbf{2 1}, 1122$

Braumann, P., Study of a particular Markov chain, Univ. Lisboa. Revista Fac. Ci. A (2) 6, 281 (1957$58)$.

M 20, 1203

Breiman, L., On transient Markov chains with application to the uniqueness problem for Markov processes, Ann. Math. Statist. 28, 499 (1957).

M 19, 468

Breiman L., Transient atomic Markov chains with a denumerable number of states, Ann. Math. Statist. 29, 212 (1958).

M 20, 595

Breny, H., Sur les "délais de passage" de certaines fonctions aléatoires de Markov, Bull. Soc. Roy. Sci. Liège 2\%, 5 (1958).

M 20, 225

- Burke, C. J., A Markovian function of a Markov chain, Ann. Math. Statist. 29, 1112 (1958).

M 21,70

Castoldi, L., Successioni stocastiche di tipo Markoviano generalizzato: le "cascate" finite di Markov, Atti Accad. Ligure 13, 45 (1957).

M 19, 988

Čerkasov, I. D., On transforming the diffusion process to a Wiener process, Teor. Veroyatnost. i Primenen. 2, 384 (1957).

M 20, 59

Cerkasov, I. D., The method of separation in the theory of Markov processes, Bul. Inst. Politehn. Iasi (N.S.) 4 (8), 23 (1958).

M 21, 442

Chacon, R. V., (See D. G. Austin) Duke Math. J. 25, 533 (1958).

Chacon, R. V., On a question of Paul Lévy, Proc. Amer. Math. Soc. 10, 460 (1959). M 21,1407

Chung, K. L., On a basic property of Markov chains, Ann. of Math. (2) 68, 126 (1958). M 20, 1204

Chung, K. L., Some aspects of continuous parameter Markov chains, Publ. Inst. Statist. Univ. Paris 6, 271 (1957).

M 21,177

Ciucu, G., Un cas d'excéption pour la répartition limite des sommes de variables aléatoires d'un processus Markoff fini, homogéne et continu. Gaz. Mat. Fiz. Ser. A 3, 141 (1958). $\quad$ M 20, 337

Cogburn, R., Termes variationnels des chaînes de Markov, C.R. Acad. Sci. Paris 24\%, 2281 (1958).

M 20, 1203
- Dantzig, D., Itérations markoviennes dans les ensembles abstraits, J. Math. Pures Appl. (9) 38, 183 (1959).

M 21, 725

Darling, D. A., Étude des fonctionnelles additives des processus markoviens. Le calcul des probabilités et ses applications, Colloq. Internat. Centre National de la Recherche Scientifique Paris LXXXVII, pp. 69-80 (1959).

M 21, 1123

- Dawson, R., Exact Markov probabilities from oriented linear graphs, Ann. Math. Statist. 28, 946 (1957).

M 20, 58

Derman, C., Non-parametric up-and-down experimentation, Ann. Math. Statist. 28, 795 (1957).

M 19, 896

Dobrushin, R. L., Central limit theorem for nonstationary Markov chains. I, Teor. Veroyatnost i Primenen. 1, 72 (1956). M 19, 184

Dobrushin, R. L., Central limit theorem for nonstationary Markov chains. II, Teor. Veroyatnost. $i$ Primenen. 1, 365 (1956).

M 20, 592

Dobrushin, R. L., An example of a countable homogeneous Markov process all states of which are instantaneous, Teor. Veroyatnost. i Primenen. 1, 481 (1956).

M 19, 691

Dobrushin, R. L., Some classes of homogeneous denumerable Markov processes, Teor. Veroyatnost. i Primenen. 2, 377 (1957).

M 20, 226

Doob, J. L., Discrete potential theory and boundaries, J. Math. Mech. 8, 433 (1959). M 21, 1083

Dynkin, E. B., Markov processes and semi-groups of operators, Teor. Veroyatnost. i Primenen. 1, 25 (1956).

M 19, 469

- Dynkin, E., Strong Markov processes, Teor. Veroyatnost. i Primenen. 1, 149 (1956).

M 19, 469

Dynkin, E. B., Infinitesimal operators of Markov processes, Teor. Veroyatnost. i Primenen. 1, 38 (1956). M 19, 691

Dynkin, E. B., Markov jump processes, Teor. Veroyatnost. i Primenen. 3, 41 (1958). M $\mathbf{2 0 ,} 59$

Dynkin, E. B., Inhomogeneous strong Markov processes, Dokl. Akad. Nauk. SSSR (N.S.) 113, 261 (1957).

M 20, 1204

Dynkin, E. B., One-dimensional continuous strong Markov processes, Teor. Veroyatnost. i Primenen. 4, 3 (1959).

M 21, 442

Dynkin, E. B., The natural topology and excessive functions connected with a Markov process, Dokl. Akad. Nauk SSSR 12\%, 17 (1959). M 21, 1122

- Egerváry, E., (See J. Aczēl) Publ. Math. Debrecen 5, 60 (1957).

Elliot, J., On an integrodifferential operator of the Cauchy type, Proc. Amer. Math. Soc. y, 616 (1956)

M 19, 185

- Elliot, J., Stochastic processes connected with harmonic functions, Trans. Amer. Math. Soc. 82, 392 (1956).

M 19, 185

Feller, W., (See J. Elliot) Trans. Amer. Math. Soc. 82, 392 (1956).

- Feller, W., A diffusion equivalent to a countable Markov chain, Proc. Nat. Acad. Sci. U.S.A. 42, 351 (1956). 
Feller, W., Boundaries induced by non-negative matrices, Trans. Amer. Math. Soc. 83, 19 (1956). M 19, 892

Feller, W., On boundaries and lateral conditions for the Kolmogorov differential equations, $A n n$. of Math. (2) 65, 527 (1957).

M 19, 892

Feller, W., Differential operators with the positive maximum property, Illinois J. Math. 3, 182 (1959).

M 21, 138

Feller, W., Notes to my paper "On boundaries and lateral conditions for the Kolmogorov differential equations.", Ann. of Math. (2) 68, 735 (1958).

M 21, 976

de Finetti, B., Sul numero di elementi al di là dell' ultimo osservato, Scritti matematici in onore di Fitippo Sibirani, pp. 95-107 (Cesare Zuffi, Bologna, 1957).

M 21, 177

Firescu, D., Sur les fonctions d'estimation des probabilités de passage d'une chaîne de Markoff, $A n$. Univ. "C. I. Parhon" Bucuresti. Ser. Sti. Nat. \%, no. 18,9 (1958).

M $\mathbf{2 0}, 1209$

Fortet, R., Résumés exhaustifs pour un processus de Markov, C.R. Acad. Sci. Paris \$47, 28 (1958).

M $\mathbf{2 0}, 225$

Freund, J. E., Some results on recurrent events, Amer. Math. Monthly 64, 718 (1957). M $\mathbf{2 0 ,} 916$

Fuchs, A., Some limit theorems for nonhomogeneous Markoff processes, Trans. Amer. Math. Soc. 86, $511,1957)$.

M 20,225

Fuchs, A., Un problème de temps d'atteinte, Publ. Inst. Statist. Univ. Paris $\mathbf{7 ,} 161$ (1958).

M 20, 1106

Gartšteln, B. N., On the limit-distribution of the maximal term for a case of homogeneous Markov chains, Užgorod. Gos. Univ. Naučn. Zap. 18, 195 (1957).

M 20,337

Getoor, R. K., Additive functionals of a Markov process, Pacific J. Math. \%, 1577 (1957).

M 20, 225

Getoor, R. K., Markov operators and their associated semi-groups, Pacific J. Math. 9, 449 (1959).

M 21,1121

- Getoor, R. K., (See R. M. Blumenthal) Pacific J. Math. 9, 399 (1959).

Gihman, I. I., A limit theorem for the number of maxima in a sequence of random variables in a Markov chain, Teor. Veroyatnost. i Primenen. 3, 166 (1958).

M 20, 464

Gilbert, E. J., On the identifiability problem for functions of finite Markov chains, Ann. Math. Statist. 30, 688 (1959).

M 21,1123

Girsanov, I. G., Some topologies connected with a Markov process, Dokl. Akad. Nauk SSSR 129, 488 (1959).

M 21, 1407

Gnedenko, B. V., On certain problems of the theory of probability, Ukrain. Mat. Ž. 9, 377 (1957).

M 19, 776

Good, I. J., (See R. Dawson) Ann. Math. Statist. 28, 946 (1957).

Goodman, L. A., Simplified runs tests and likelihood ratio tests for Markoff chains, Biometrika 45, 181 (1958).

M 19, 1090
Goodman, L. A., Exact probabilities and asymptotic relationships for some statistics from $m$-th order Markov chains, Ann. Math. Statist. 29, 476 (1958).

M 20, 225

Goodman, L. A., Asymptotic distributions of "psisquared" goodness of fit criteria for $m$-th order Markov chains, Ann. Math. Statist. 99, 1123 (1958).

M 20, 1022

Goodman, L. A., On some statistical tests for $m$ th order Markov chains, Ann. Math. Statist. 30, 154 (1959).

M 21, 78

Goodman, L. A., A note on Stepanow's tests for Markov chains, Teor. Veroyatnost. i Primenen. 4, 93 (1959).

M $\mathbf{2 1}, 322$

Hájek, J., Remark on the article "On certain sequences of sets of points on a circle", Časopis Pěst. Mat. 81, 77 (1956).

M 19, 134

Hajnal, J., Weak ergodicity in non-homogeneous Markov chains, Proc. Cambridge Philos. Soc. 54, 233 (1958).

M 20, 463

Hammersley, J. M., Markovian walks on crystals, Compositio Math. 11, 171 (1953).

M 19, 71

Harris, T. E., The random functions of cosmic-ray cascades, Proc. Nat. Acad. Sci. U.S.A. 43, 509 (1957).

M 19, 328

Harris, T. E., Transient Markov chains with stationary measures, Proc. Amer. Math. Soc. 8, 937 (1957).

M 19, 989

Has'minskiǔ, R. Z., Diffusion processes and elliptic equations degenerating at the boundary of a region, Teor. Veroyatnost. i Primenen. 3, 430 (1958).

M 21, 71

Hennequin, P., Processus en cascade à $n$ dimensions et problèmes de moments, C.R. Acad. Sci. Paris 24\%, 857 (1958).

M 20, 916

Hille, E., Perturbation methods in the study of Kolmogoroff's equations, Proc. Int. Cong. Math. 3, 365 (North-Holland Publishing Co., Amsterdam, 1956).

M 19, 327

Homma, T., On some fundamental traffic problems, Yokohama Math. J. 5, 99 (1957).

M 20, 594

Hunt, G. A., Markoff processes and potentials. I, II., Illinois J. Math. 1, 44 and 316 (1957). M 19. 951

Hunt, G. A., Markoff processes and potentials, Proc. Nat. Acad. Sci. U.S.A. 42, 414 (1956).

M 19, 1089

Hunt, G. A., Markoff processes and potentials. III., Illinois J. Math. 2, 151 (1958).

M 21, 1082

Il'yašenko, A. A., Des lois asymptotiques pour les chaînes de Markoff avec un nombre fini d'états, Ukrain. Mat. Z. 10, no. 1, 23 (1958).

M 20, 812

Jacobs, K., Fastperiodische diskrete Markoffsche Prozesse von endlicher Dimension, Abh. Math. Sem. Univ. Hamburg $\mathbf{1 1}, 194$ (1957).

M 19, 778

Jacobs, K., Markoffsche Prozesse mit monomialer Selbststeuerung., Arch. Math. 8, 298 (1957).

M 19, 988 
Jacobs, K., Zur Theorie der Markoffschen Prozesse, Math. Ann. 133, 375 (1957).

M 19, 1089

Jacobs, K., Fastperiodische Markoffsche Prozesse, Math. Ann. 134, 408 (1958).

M 20, 595

Jacobs, K., Konjunkturschwankungen Markoffscher $n$-Personen-Prozesse mit monomialer Regelung, Math. Z. 69, 247 (1958).

M 20, 716

Jiřina, M., The asymptotic behaviour of branching stochastic processes, Czechoslovak Math. J. $\mathbf{g}(\mathbf{8 2})$, 130 (1957).

M 20, 225

Jiřna, M., Stochastic branching processes with continuous state space, Crechoslovak Math. J. 8 (83), 292 (1958).

M 21, 69

- Jushkevich, A., (See E. Dynkin) Teor. Veroyatnost. i Primenen. 1, 149 (1956).

Kac, M., A class of limit theorems, Trans. Amer. Math. Soc. 84, 459 (1957).

M 19, 184

Kanngiesser, W., Grenzwertsätze für verschwindende Übergangswahrscheinlichkeiten, Mitteilungsbl. Math. Statist. 8, 15, 141, and 177 (1956).

M 19, 989

- Karlin, S., The classification of birth and death processes, Trans. Amer. Math. Soc. 86, 366 (1957).

M $\mathbf{2 0}, 226$

- Karlin, S., A characterization of birth and death processes, Proc. Nat. Acad. Sci. U.S.A. 45, 375 (1959).

M 21, 177

Kawamura, T., On Markov chains. I., Rep. Statist. Appl. Res. Un. Jap. Sci. Enars. 4, 111 (1957).

M 19, 778

Kemeny, J. G., Markov processes in learning theory, Psychometrika 22, 221 (1957).

- Kemeny, J. G., Semimartingales of Markov chains, Ann. Math. Statist. 29143 (1958).

M 20, 58

- Kendall, D. G., The calculation of the ergodic projection for Markov chains and processes with a countable infinity of states, Acta Math. 9\%, 103 (1957).

M 19, 469

Kendall, D. G., A totally unstable denumerable Markov process, Quart. J. Math. Oxford Ser. (2) 9, 149 (1958). M 20, 716 Kendall, D. G., (See D. Vere-Jones) Teor. Veroyatnost. i Primenen. 4, 97 (1959).

Kennedy, M., A convergence theorem for a certain class of Markoff processes, Pacific J. Math. \%, 1107 (1957).

M 19, 988

Kinney, J. R., Singular functions associated with Markov chains, Proc. Amer. Math. Soc. 9, 603 (1958).

M 20, 1019

- Koopmans, L., (See D. Blackwell) Ann. Math. Statist. 28, 1011 (1957).

Kosmák, L., On certain sequences of sets of points on a circle, Casopis Pést. Mat. 80, 299 (1955).

M 19, 134

Kozin, F., On the quadratic detector. I., J. Math. Phys. 38, 119 (1959-60).

M 21, 1237

Koźniewska, I., Ergodicity of non-homogeneous Markov chains with two states, Colloq. Math. 5, 208 (1958).

M 20, 1203

Kramer, H. P., Symmetrizable Markov matrices, Ann. Math. Statist. 30, 149 (1959).
- Krishna Iyer, P. V., Cumulants of some distributions arising from a two-state Markoff chain, Proc. Cambridge Philos. Soc. 55, 273 (1959). M 21, 725

- Lamperti, J., Chains of infinite order and their application to learning theory, Pacific J. Math. 9, 739 (1959)

M 21, 1407

Leipnik, R., Integral equations, biorthonormal expansions, and noise, J. Soc. Indust. Appl. Math. g, 6 (1959)

M 20, 1203

Lévy, P., Processus semi-markoviens, Proc. Int. Cong. Math. 3, 416 (North-Holland Publ. Co., Amsterdam, 1956.)

M 19, 469

Lévy, P., Remarques sur le processus de W. Feller et H. P. McKean, C.R. Acad. Sci. Paris 245, 1772 (1957).

M 19, 1202

Lévy, P., Processus strictement markoviens, C.R. Acad. Sci. Paris 246, 1490 (1958).

M $\mathbf{2 0}, 463$

Lévy, P., Fonctions linéairement markoviennes d'ordre n, Math. Japon 4, 113 (1957) M 20, 1203

Lévy, P., Processus markoviens et stationnaires. Cas dénombrable, Ann. Inst. H. Poincaré 16, 7 (1958).

M 21, 71

Lévy, P., Construction du processus de W. Feller et H. P. Mckean en partant du mouvement Brownien. The Harald Cramér Volume, pp. 162174. (Almqvist \& Wiksell, Stockholm; John Wiley \& Sons, New York, 1959.) M 21237

Madansky, A., Least squares estimation in finite Markov processes, Psychometrika 24, 137 (1959).

M 21, 183

Mandl, P., Sur le comportement asymptotique des probabilitiés dans les ensembles des états d'une chaîne de Markov homogène, Časopis Pěst. Mat. 84, 140 (1959).

M 21, 1407

Martynov, A. V., On local infinite divisibility of Markoff processes, Dokl. Akad. Nauk SSSR (N.S.) 113, 752 (1957).

M 20, 1204

- Maruyama, G., Some properties of one-dimensional diffusion processes, Mem. Fac. Sci. Kyusyu Univ. Ser. A. Math. 11, 117 (1957). M 20, 595

Maruyama, G., On the strong. Markov property, Mem. Fac. Sci. Kyushu Univ., Ser. A 13, 17 (1959).

M 21, 1123

- Maslov, K. V., On infinitesimal operators of a class of Markov processes, Teor. Veroyatnost. $i$ Primenen. 3, 70 (1958).

M $\mathbf{2 0}, 59$

Mauldon, J. G., On non-dissipative Markov chains, Proc. Cambridge Philos. Soc. 53, 825 (1957).

M 19, 1089

- McGregor, J., (See S. Karlin) Trans. Amer. Math. Soc. 86, 366 (1957).

- McGregor, J., (See S. Karlin) Proc. Nat. Acad. Sci. U.S.A. 45, 375 (1959).

- McKean, H. P., (See W. Feller) Proc. Nat. Acad. Sci. U.S.A. 42, 351 (1956).

Mešalkin, L. D., Limit theorems for Markov chains with a finite number of states, Teor. Veroyatnost. $i$ Primenen. 3, 361 (1958).

M 21, 441

Miehle, W., Calculation of higher transitions in a Markov process, Operations Res. 6, 693 (1958).

M 20, 716 
Mihoc, G., Fonctions d'estimation efficients pour les suites de variables dépendantes, Bull. Math. Soc. Sci. Math. Phys. R. P. Roumaine (N.S.) 1 (49), 449 (1957).

M 21,726

Moran, P. A. P., The suvival of a mutant gene under selection, J. Austral. Math. Soc. 1, 121 (1959-61).

M 21, 830

Mott, J. L., Conditions for the ergodicity of nonhomogeneous finite Markov chains, Proc. Roy. Soc. Edinburgh. Sect. A 64, 369 (1957).

M 19, 988

- Mott, J. L., Matrix norms applied to weakly ergodic Markov chains, Arch. Math. 8, 331 (1957).

M 19, 988

Moyal, J. E., Discontinuous Markoff processes, Acta Math. 98, 221 (1957).

M 20, 59

Nagaev, S. V., Some limit theorems for homogeneous Markoff chains, Dokl. Acad. Nauk SSSR (N.S.) 115, 237 (1957).

M $\mathbf{2 0}, 58$

Nagaev, S. V., Some limit theorems for stationary Markov chains, Teor. Veryoyatnost. i Primenen. 2, 389 (1957).

M 20, 224

Nelson, E., Representation of a Markovian semigroup and its infinitesimal generator, J. Math. Mech. y, 977 (1958).

M R0, 1184

Nelson, E., The adjoint Markoff process, Duke Math. J. 25, 671 (1958).

M 21, 70

Neveu, J., Sur le comportement asymptotique des chaînes de Markov, C.R. Acad. Sci. Paris $\mathbf{2 4 5}, 493$ (1957).

M 19, 778

Neveu, J., Théories des semi-groupes de Markov, Univ. California Publ. Statist. 2, 319 (1958).

M $\mathbf{2 1 ,} 293$

Neveu, J., Processus sous-markoviens stationnaires, C.R. Acad. Sci. Paris 249, 1447 (1959). M M1, 1123

Neveu, J., Sur le comportement asymptotique des chaînes de Markov, Colloq. Internat'l. du Centre National de la Recherche Scientifique Paris LXXXVII, pp. 185-193 (1959). M $\quad$ 21, 1236

Neveu, J., Un exemple de processus markoviens stationnaires, C.R. Acad. Sci. Paris 249, 1309 (1959).

M 21,1237

Nisio, M., Note on random Riemann sum, J. Math. Soc. Japan 9, 448 (1957).

M $\mathbf{2 0}, 337$

Nomoto, H, Continuity of strong Markov processes, Sûgaku 9, 15 (1957-58).

M 20, 337

Onicescu, O., La répartition limite des sommes de variables aléatoires d'un processus Markov fini, homogène et continuu, An. Univ. "C. I. Parhon" Bucuresti. Ser. Sti. Nat. 5, no. 12, 9 (1956).

M 20, 337

Patil, V. T., The consistency and adequacy of the Poisson-Markoff model for density fluctuations, Biometrika 44, 43 (1957).

M 19, 468

- Postnikov, A. G., On Bernoulli-normal sequences of symbols, Izv. Akad. Nauk SSSR. Ser. Mat. 21, 501 (1957).

M 21, 123

Postnikov, A. G., A Markov-sequence of symbols and a normal continued fraction, Izv. Akad. Nauk SSSR. Ser. Mat. 21, 729 (1957).
Povzner, A. Ya., (See K. V. Maslov) Teor. Veroyatnost. i Primenen. 3, 70 (1958).

Prabhu, N. U., Some exact results for the finite dam, Ann. Math. Statist. 29, 1234 (1958). M 20, 1019

Prékopa, A., On additive and multiplicative totals, Acta Math. Acad. Sci. Hungar. 8, 107 (1957). M 19, 399 Pyateckiı̌, I. I., (See A. G. Postnikov) Izv. Akad. Nauk SSSR. Ser. Mat. 21, 501 (1957).

- Pyateckiř, I. I., (See A. G. Postnikov) Izv. Akad. Nauk SSSR. Ser. Mat. 21, 729 (1957).

Radu, N. C., Une propriété asymptotique des densités d'une chaîne de Markov continue au cours du temps, Com. Acad. R.P. Romîne ', 929 (1957).

M $\mathbf{2 0}, 337$

Ramakrishnan, A., Ergodic properties of some simple stochastic processes, Z. Angew. Math. Mech. 37, 336 (1957).

M 19, 778

Ray, D., Stationary Markov processes with continuous paths, Trans. Amer. Math. Soc. 8\%, 452 (1956).

M 21,316

Ray, D., Stable processes with an absorbing barrier, Trans. Amer. Math. Soc. 89, 16 (1958). M 21, 726

Ray, D., Resolvents, transition functions, and strongly Markovian processes, Ann. of Math. (2) \%0, 43 (1959).

M 21, 1122

- Reuter, G. E. H., (See D. G. Kendall) Acta Math. 97, 103 (1957).

Reuter, G. E. H., Denumerable Markov processes and the associated contraction semigroups on $l$, Acta Math. 9\%, 1 (1957).

M 21, 176

Reuter, G. E. H., Denumerable Markov processes. II., J. London Math. Soc. 34, 81 (1959). M $\mathbf{2 1 , ~} 176$

Rosenblatt, D., On linear models and the graphs of Minkowski-Leontief matrices, Econometrica 25, 325 (1957).

M 19, 617

- Rosenblatt, M., (See C. J. Burke) Ann. Math. Statist. 29, 1112 (1958).

Rosenblatt, M., Functions of a Markov process that are Markovian, J. Math. Mech. 8, 585 (1959).

M 21, 442

Rózsa, P., Remarks on the spectral decomposition of a stochastic matrix, Magyar Tud. Akad. Mat. Fiz, Oszt. Közl. \%, 199 (1957). M 20,523

- Rubin, H., (See A. T. Bharucha-Reid) Proc. Nat. Acad. Sci. U.S.A. 44, 1057 (1958).

Sack, R. A., Equivalence of two absorption problems with Markovian transitions and continuous or discrete time parameters, Proc. Cambridge Philos. Soc. 55, 177 (1959).

M 21, 579

Sacks, J., Asymptotic distribution of stochastic approximation procedures, Ann. Math. Statist. 29, 373 (1958).

M 20, 811

Sankaranarayanan, G., Some asymptotic properties of Poisson process, Tôhoku Math. J. (2) 10, 60 (1958). M 20, 337

Sarymsakov, T. A., On inhomogeneous Markoff chains, Dokl. Akad. Nauk SSSR 120, 465 (1958).

M 20, 916

- Schneider, H., (See J. L. Mott) Arch. Math. 8, 331 (1957). 
Sevastjanov, B. A., An ergodic theorem for Markov processes and its application to telephone systems with refusals, Teor. Veroyatnost. i Primenen. 2, 106 (1957).

M 19, 988

Shakuntala, N. S., (See P. V. Krishna Iyer) Proc. Cambridge Philos. Soc. 55, 273 (1959).

Skorohod, A. V., Limit theorems for Markov processes, Teor. Veroyatnost. i Primenen. 3, 217 (1958.)

M 21, 71

Snell, J. L., (See J. G. Kemeny) Psychometrika 22, 221 (1957).

Snell, J. L., (See J. G. Kemeny) Ann. Math. Statist. 29, 143 (1958).

Snell, J. L., Finite Markov chains and their applications, Amer. Math. Monthly 66, 99 (1959).

M 20, 1019

Statulyavičus, V. A., On a local limit theorem for inhomogeneous Markoff chains, Dokl. Akad. Nauk SSSR N.S.) 10\%, 516 (1956).

M 19, 71

Statulyavičus, V. A., Asymptotic expansion for inhomogeneous Markov chains, Dokl. Akad. Nauk SSSR (N.S.) 11\%, 206 (1957).

M 19, 185

Statulyavičus, V. A., A local limit theorem for nonhomogeneous Markoff chains with an enumerable number of possible states, Dokl. Akad. Nauk SSSR (N.S.) 115, 872 (1957).

M 20, 462

Suppes, P., (See J. Lamperti) Pacific J. Math. 9, 739 (1959).

Šur, M. G., Ergodic properties of invariant Markov chains on homogeneous spaces, Teor. Veroyatnost. i Primenen. 3, 137 (1958).

M 20, 464

Takács, L., On a probability problem in theory of counters, Ann. Math. Statist. 29, 1257 (1958).

M 20, 1018

Takashima, M., Note on evolutionary processes, Bull. Math. Statist. 7, 18 (1956).

M 19, 327

Tanaka, H., (See G. Maruyama) Mem. Fac. Sci. Kyusyu Univ. Ser. A. Math. 11, 117 (1957).

Tanaka, H., Certain limit theorems concerning onedimensional diffusion processes, Mem. Fac. Sci. Kyusyu Univ. Ser. A. Math. 12, 1 (1958).

M 20, 595

Tsurumi, S., On general ergodic theorems. II., Tôhoku Math. J. (2) 9, 1 (1957).

M 20, 398

Tsurumi, S., On the ergodic theorems concerning Markov processes, Tôhoku Math. J. (2) 10, 146 (1958).

M 20, 1160

Ueno, T., On a method for investigation of sequences of dependent stochastic variables, Sûgaku 8, 16 (1956/57).

M 20, 594

Ueno, T., On a method for investigation of sequences of dependent stochastic variables. II., Sûgaku 8, $83(1956 / 57)$.

M 20, 594

Ueno, T., Some limit theorems for temporally discrete Markov processes. II, J. Fac. Sci. Univ. Tokyo. Sect. I. \%, 557 (1958).

M 20, 594 Urbanik, K., Remarks on invariant functions in Markov processes, Colloq. Math. 5, 223 (1958).

M 20, 1204

Urbanik, K., Limit properties of Markoff processes, Rozprawy Mat. 13, 46 pp. (1957).
- Vere-Jones, D., A commutativity problem in the theory of Markov chains, Teor. Veroyatnost. i Primenen. 4, 97 (1959).

M 21, 316

Volkonskiľ, V. A., A multidimensional limit theorem for Markov chains with a countable set of states, Teor. Veroyatnost i Primenen. 2, 230 (1957).

M 19, 1089

Volkonskiǔ, V. A., Random substitution of time in strong Markov processes, Teor. Veroyatnost. i Primenen. 3, 332 (1958).

M 20, 1203

Volkov, I. S., On the distribution of sums of random variables defined on a homogeneous Markov chain with a finite number of states, Teor. Veroyatnost. $i$ Primenen. 3, 413 (1958).

M 21, 442

Watanabe, T., Some general properties of Markov processes, J. Inst. Polytech. Osaka City Univ., Ser. A 10, 9 (1959).

M 21, 1237

Waugh, W. A. O'N., Conditioned Markov processes, Biometrika 45, 241 (1958). M 19, 1089

Whittle, P., Some conbinatorial results for matrix powers, Quart. J. Math. Oxford Ser. (2) 2\%, 316 (1956).

M 19, 832

Whittle, P., On the use of the normal approximation in the treatment of stoichastic processes, J. Roy. Statist. Soc., Ser. B 19, 268 (1957). M 2178

Wolff, K-H., Die Ruinwahrscheinlichkeit in der kollektiven Risikotheorie für spezielle Schadensverteilungen, Statist. Vierteljschr. 10, 163 (1957).

M 21,1237

Zítek, F., On estimating transition probabilities, $A p l$. Mat. 2, 251 (1957).

M 19, 778

-Zoutendijk, G., (See D. van Dantzig) J. Math. Pures Appl. (9) 38, 183 (1959).

\section{Stationary Processes}

Akutowicz, E. J., On an explicit formula in linear least squares prediction, Math. Scand. 5, 261 (1957).

M 20, 716

Bass, J., Contribution à l'étude de certaines fonctions susceptibles de représenter la vitesse d'un fluide turbulent, J. Math. Pures Appl. (9) 3\%, 173 (1958).

M 21, 316

Bass, J., Sur la définition temporelle des fonctions aléatoires, Publ. Inst. Statist. Univ. Paris 6, 199 (1957).

M 21, 317

- Bass, J., Sur les fonctions pseudo-aléatoires, C.R. Acad. Sci. Paris 24\%, 1083 (1958) M 21, 317

Bass, J., Fonctions pseudo-aléatoires et fonctions de Wiener, C.R. Acad. Sci. Paris 24\%, 1163 (1958).

M $\mathbf{2 1}, 317$

Belyaev, Yu. K., On the unboundedness of the sample functions of Gaussian processes, Teor. Veroyatnost. i Primenen. 3, 351 (1958).

M 20, 1205

Bertrandias, J. P., Formation d'une classe de fonctions pseudo-aléatoires, C.R. Acad. Sci. Paris $\mathbf{2 4 8 ,}$ 513 (1959).

M 21, 317

Blanc, C., Calcul numérique et considérations stochastiques, Proc. Symp. on Modern Computers, Rome, October 1956, pp. 23-26. (Libreria Eredi Virgilio Veschi, Rome.) 
Campbell, L. L., On the use of Hermite expansions in noise problems, J. Soc. Indust. Appl. Math. 5, 244 (1957).

M 19, 1098

Cheng, S. L., On the values at the fixed moment of strictly stationary generalized stochastic processes, Sci. Record (N.S.) R, 47 (1958). M 21, 1124

Chiang, T. P., Extrapolation theory of a homogeneous random field with continuous parameters, Teor. Veroyatnost. i Primenen. 2, 60 (1957).

M 20, 717

Chover, J., On positive-definite integral kernels and a related quadratic form, Trans. Amer. Math. Soc. 89, 92 (1958).

M 20, 792

Davenport, W. B., An introduction to the theory of random signals and noise, (McGraw-Hill Book Co., Inc., New York-Toronto-London, 1958).

M 19, 1090

Diederich, F. W., Expected number of maxima and minima of a stationary random process with nonGaussian frequency distribution, NACA Tech. Note 3\%18, 21 pp. (1957).

M 19, 470

Drenick, R., A non-linear prediction theory, Trans. I.R.E., PGIT-4, 146 (1954).

M 19, 516

- Feldman, J., (See J. Chover) Trans. Amer. Math. Soc. 89, 92 (1958).

M 20, 792

- Freiberger, W., Approximate distributions of noise power measurements, Quart. Appl. Math. 1\%, 271 (1959).

M 21, 1131

\ Freund, J. E., (See I. Miller) J. Appl. Phys. 2\%, 1290 (1956).

Gladyšev, E. G., On multi-dimensional stationary random processes, Teor. Veroyatnost. i Primenen. 3, 458 (1958).

M 21, 178

Grenander, U., Modern trends in time series analysis, Sankhyã 18, 149 (1957).

M 19, 1206

Grenander, U., Bandwidth and variance in estimation of the spectrum, J. Roy. Statist. Soc., Ser. B 20, 152 (1958).

M 20, 468

$\checkmark$ Grenander, U., (See W. Freiberger) Quart. Appl. Math. 1\%, 271 (1959).

Gudzenko, L. I., Small fluctuations in an essentially nonlinear autocorrelation system, Dokl. Akad. Nauk SSSR 125, 62 (1959).

M 21, 1417

Hájek, J., Predicting a stationary process when the correlation function is convex, Czechoslovak Math. J. 8 (83), 150 (1958).

M $\mathbf{2 0}, 337$

Hannan, E. J., The variance of the mean of a stationary process, J. Roy. Statist. Soc., Ser. B 19, 282 (1957).

M 19, 1098

- Helson, H., Prediction theory and Fourier series in several variables, Acta Math. 99, 165 (1958).

M 20, 691

Ibraigimov, I. A., Some limit theorems for stochastic processes stationary in the strict sense, Dokl. Akad. Nauk SSSR 125, 711 (1959).

M 21, 726
- Kendall, D. G., The calculation of the ergodic projection for Markov chains and processes with a countable infinity of states, Acta Math. 9\%, 103 (1957).

M 19, 469

Koronkevič, A. I., Some remarks on evaluating the accuracy of linear extrapolation and filtration, Teor. Veroyatnost. i Primenen. 2, 116 (1957).

M 19, 893

Koronkevich, A. E., The ergodic theorem for nonstationary random functions, Dopovidi Akad. Nauk Ukrain. RSR 195\%, 231 (1957). M 19, 1203

- Krée, P., (See J. Bass) C.R. Acad. Sci. Paris $\mathbf{2 4 7}$ 1083 (1958).

Lomnicki, Z. A., On some moments and distributions occurring in the theory of linear stochastic processes. I., Monatsh. Math. 61, 318 (1957).

M 19, 1090

Longo, A., Un criterio di previsione di Wiener nella statistica, Univ. e Politec. Torino. Rend. Sem. Mat. 1\%, 7 (1957-58).

M 21, 579

Longuet-Higgins, M. S., The statistical analysis of a random, moving surface, Philos. Trans. Roy. Soc. London, Ser. A 249, 321 (1957). $\quad$ M 19, 328

- Lowdenslager, D., (See H. Helson) Acta Math. 99, 165 (1958).

- Masani, P., (See N. Wiener) Acta Math. 98, 111 (1957).

- Masani, P., (See N. Wiener) Acta Math. 99, 93 (1958).

- Masani, P., (See N. Wiener) C.R. Acad. Sci. Paris 246, 1492 (1958).

- Masani, P., (See N. Wiener) C.R. Acad. Sci. Paris 246, 1655 (1958).

Masani, P., Sur les processus vectoriels minimaux de rang maximal, C.R. Acad. Sci. Paris 246, 2215 (1958).

M 20, 717

Masani, P., Sur la prévision linéaire d'un processus vectoriel à densité spectrale non bornée, C.R. Acad. Sci. Paris 246, 2337 (1958). $\quad$ M 20,717

- Masani, P., Non-linear prediction, The Harald Cramér volume, Probability and Statistics (edited by Ulf Grenander), pp. 190-212. (Almqvist \& Wiksell, Stockholm; John Wiley \& Sons, New York; 1959.)

M 21, 1407

Mihoc, G., Sur les lois limites des variable vectorielles enchaînées au sens de Markoff, Teor. Veroyatnost. i Primenen. 1, 103 (1956). M 19, 893

- Miller, I., Some results on the analysis of random signals by means of a cut-counting process, $J$. Appl. Phys. 2\%, 1290 (1956). M 19, 72

Moy, S. C., Successive recurrence times in a stationary process, Ann. Math. Statist. 30, 1254 (1959).

M 21, 1408

Nelson, E., A functional calculus using singular Laplace integrals, Trans Amer. Math. Soc. 88, 400 (1958).

M 20, 436

Parasiouk, O. S., Sur le problème de la filtration des processus stationnaires généralisés, Ukrain. Mat. Ž. 9, 210 (1957).

M 19, 892 
Parzen, E., On consistent estimates of the spectrum of a stationary time series, Ann. Math. Statist. 28, 329 (1957).

M 19, 587

Parzen, E., On choosing an estimate of the spectral density function of a stationary time series, Ann. Math. Statist. 28, 921 (1957).

M 20, 341

Pinsker, M. S., Extrapolation of homogeneous stochastic fields and amount of information about a Gaussian field contained in another Gaussian stochastic field, Dokl. Akad. Nauk SSSR (N.S.) 112, 815 (1957).

M 19, 470

- Reuter, G. E. H., (See D. G. Kendall) Acta Math. 97, 103 (1957).

- Root, W. L., (See W. B. Davenport) (McGrawHill Book Co., Inc., New York-Toronto-London, 1958).

Rosenblatt, M., Some purely deterministic processes, J. Math. Mech. 6, 801 (1957).

M 20, 59

Rosenblatt, M., The multidimensional prediction problem, Proc. Nat. Acad. Sci. U.S.A. 43, 989 (1957).

M $\mathbf{2 0}, 716$

Scheidegger, A. E., The random-walk model with auto-correlation of flow through porous media, Canad. J. Phys. 36, 649 (1958).

M 19, 1090

Silverman, R. A., Locally stationary random processes, Div. Electromag. Res., Inst. Math. Sci., New York Univ., Res. Rep. MME-2, 8 pp. (1957).

M 19, 893

Tick, L. J., A non-linear random model of gravity waves. I., J. Math. Mech. 8, 643 (1959). M $\mathbf{2 1 ,} 860$ Tsian, T., On linear extrapolation of a discrete homogeneous stochastic field, Dokl. Akad. Nauk SSSR (N.S.) 112, 207 (1957).

M 19, 327

Urbanik, K., Generalized stochastic processes, Studia Math. 16, 268 (1958).

M 20, 715 Urbanik, K., Filtering of stationary generalized stochastic processes, Sci. Record (N.S.) 2, 43 (1958).

M 21, 1124

\ Urbanik, K., (See S. L. Cheng) Sci. Record (N.S.) 2, 47 (1958).

Urbanik, K., The conditional expectations and the ergodic theorem for strictly stationary generalized stochastic processes, Studia Math. 1\%, 267 (1958).

M 21, 1124

Watanabe, T., A remark on an additive process, Sûgaku 8, 215 (1956/57).

M 20, 338

Wever, F., Über stationäre Null-Eins-Prozesse mit beschränkten Lücken, Math. Z. \%1, 283 (1959).

M 21, 1408

Whittle, P., On the variation of yield variance with plot size, Biometrika 43, 337 (1956). M 19, 72

- Wiener, N., The prediction theory of multivariate stochastic processes. I. The regularity condition, Acta Math. 98, 111 (1957).

M 20, 716

- Wiener, N., The prediction theory of multivariate stochastic processes. II. The linear predictor, Acta Math. 99, 93 (1958).

M 20, 717
- Wiener, N., Sur la prévision linéaire des processus stochastiques vectoriels à densité spectrale bornée, C.R. Acad. Sci. Paris 246, 1492 (1958). M 20, 717

- Wiener, N., Sur la prévision linéaire des processus stochastiques vectoriels à densité spectrale bornée (détermination de la fonction génératrice). C.R. Acad. Sci. Paris 246, 1655 (1958). $\quad$ M 20, 717

Wiener, N., Nonlinear problems in random theory (John Wiley \& Sons, Inc., New York; Chapman \& Hall, Ltd., London; 1958).

M 20, 1201

- Wiener N., (See P. Masani) The Harald Cramér volume, Probability and Statistics (edited by Ulf Grenander), pp. 190-212. (Almqvist \& Wiksell, Stockholm; John Wiley \& Sons, New York; 1959.)

Zaremba, S. K., (See Z. A. Lomnicki) Monatsh. Math. 61, 318 (1957).

Zítek, F., Sur la durée des processus linéaires, Czechoslovak Math. J. 8 (83), 122 (1958).

M 20, 595

\section{Special Processes}

Akaike, H., On the statistical control of the gap process, Ann. Inst. Statist. Math., Tokyo, 10, 233 (1959).

Akutowicz, E. J., (See N. Wiener) Rend. Circ. Mat. Palermo (2) 6, 205 (1957).

Bahadur, R. R., A note on the fundamental identity of sequential analysis, Ann. Math. Statist. 29, 534 (1958).

M 20, 341

Bailey, N. T. J., The mathematical theory of epidemics (Hafner Publishing Co., New York, 1957).

M $\mathbf{2 0}, 261$

Bartlett, M. S., On theoretical models for competitive and predatory biological systems, Biometrika 44, 27 (1957).

M 19, 233

Bartlett, M. S., Some problems associated with random velocity, Publ. Inst. Statist. Univ. Paris 6, 261 (1957).

M 20, 1106

Batschelet, E., Zur Theorie der wiederkehrenden Ereignisse. I., Arch. Math. 8, 294 (1957).

M 19, 1091

Batschelet, E., Zur Theorie der wiederkehrenden Ereignisse. II., Arch. Math. 8, 184 (1957).

M 19, 1091

Bellman, R., Random walk, scattering, and invariant imbedding. I. One-dimensional discrete case, Proc. Nat. Acad. Sci. U.S.A. 43, 930 (1957).

M 20, 1019

- Bernstein, R. I., (See K. S. Miller) Quart. Appl. Math. 16, 137 (1958).

Bertotti, B., Trasformazioni di coordinate e movimento browniano, Boll. Un. Mat. Ital. (3) 13, 217 (1958).

M 20, 1106

- Birkhoff, G., Kinematics of homogeneous turbulence, J. Math. Mech. ', 663 (1958). M 20,830

- Birkhoff, G., Reactor criticality and nonnegative matrices, J. Soc. Indust. Appl. Math. 6, 354 (1958).

M $\mathbf{2 0}, 1213$ 
Birkhoff, G., Reactor criticality in transport theory, Proc. Nat. Acad. Sci. U.S.A. 45, 567 (1959).

M 21, 592

Blanc-Lapierre, A., Méthodes mathématiques de la mécanique statistique (Masson et Cie, Paris, 1959).

M 21, 453

Blumenson, L. E. (See K. S. Miller) Quart. Appl. Math. 16, 137 (1958).

Borovkov, A. A., Some problems concerned with large deviations of the maximum of sums of independent equally distributed random variables, Dokl. Akad. Nauk SSSR 121, 13 (1958).

M 20, 1106

Breitenberger, E., Theory of multiple scattering, Proc. Roy. Soc. London, Ser. A 250, 514 (1959).

M 21, 212

Broadbent, S. R., Percolation processes. I. Crystals and mazes, Proc. Cambridge Philos. Soc. 53, 629 (1957)

M 19, 989

- Burke, C. J., A component model for stimulus variables in discrimination learning, Psychometrika 22, 133 (1957).

M 19, 106

Carleson, L., A mathematical model for highway traffic, Nordisk Mat. Tidskr. 5, 176 (1957).

M 20, 1106

- Casal, P., (See A. Blanc-Lapierre) (Masson et Cie, Paris, 1959).

Čencov, N. N., Le mouvement brownien à plusieurs paramètres de M. Lévy et le bruit blanc généralisé, Teor. Veroyatnost. i Primenen. 2, 281 (1957).

M 20, 227

Cerkasov, I. D., On the equations of Kolmogorov, Uspehi Mat. Nauk (N.S.) 12, no. 5, 237 (1957).

M 19, 1091

$\checkmark$ Chaudhuri, A. K., (See S. S. Jogdeo) Proc. Sec. Cong. on Theoretical and Applied Mechanics, New Delhi, October, 1956, pp. 237-252. (Indian Inst. of Tech., Kharagpur.)

$\checkmark$ Christie, L. S., (See H. White) Operations Res. 6, 79 (1958).

Cistyakov, V. P., Local limit theorems for branching processes, Teor. Veroyatnost. i Primenen. 2, 360 (1957).

M 19, 1092

Cistyakov, V. P., Generalization of a theorem for branching processes, Teor. Veroyatnost. i Primenen. 4, 109 (1959).

M 21, 178

- Consael, R., (See A. Lamens) Acad. Roy. Belg. Bull. Cl. Sci. (5) 43, 597 (1957).

Cramér, H., Collective risk theory: A survey of the theory from the point of view of the theory of stochastic processes, Reprinted from Försäkringsaktiebolaget Skandia. (Skandia Insurance Co., Stockholm, 1955.)

M 19, 779

Daboni, L., Osservazioni a proposito del problema del rinnovamento, Giorn. Ist. Ital. Attuari 19, 44 (1956).

M 19, 470

Datzeff, A. B., Über einen Fall der Molekülbewegung, C. R. Acad. Bulgare Sci. 12, 105 (1959).

M 21, 977
David, H. T., A three-sample Kolmogorov-Smurnov test, Ann. Math. Statist. 29, 842 (1958).

M 20, 66

Doig, A., A bibliography on the theory of queues, Biometrika 44, 490 (1957).

M 19, 692

- Domb, C., On random walks with restricted reversals, Proc. Cambridge Philos. Soc. 54, 48 (1958).

M 19, 1203

Doob, J. L., Interrelations between Brownian motion and potential theory, Proc. Int. Cong. Math. 3, 202 (North-Holland Publishing Co., Amsterdam, 1956).

M 20, 338

Doob, J. L., A new look at the first boundary-value problem, Proc. Symp. in Appl. Math. 7, 21. (McGraw-Hill Book Co., New York-TorontoLondon, 1957.)

M 19, 1091

Doob, J. L., Brownian motion on a Green space, Teor. Veroyatnost. i Primenen. 2, 3 (1957).

M 21, 976

Doob, J. L., La théorie des probabilitiés et le premier problème des fonctions frontières, Publ. Inst. Statist. Univ. Paris 6, 289 (1957).

M 21, 977

Doob, J. L., Probability theory and the first boundary value problem, Illinois J. Math. 2, 19 (1958).

М 21, 977

- Driml, M., Trois théorèmes concernant l'expèrience dans le cas continu, C.R. Acad. Sci. Paris 248, 629 (1959).

M 20, 1206

- Dvoretzky, A., Triple points of Brownian paths in 3-space, Proc. Cambridge Philos. Soc. 53, 856 (1957).

M 20, 226

Epstein, B., The existence of a generalized Green's function in the plane, J. Analyse Math. 4, 222 $(1955 / 56)$.

M 19, 406

- Erdos, P., (See A. Dvoretzky) Proc. Cambridge Philos. Soc. 53, 856 (1957).

- Estes, W. K., (See C. J. Burke) Psychometrika 22, 133 (1957).

Fabian, V., Zufälliges Abrunden und die Konvergenz des linearen (Seidelschen) Iterationsverfahrens, Math. Nachr. 16, 265 (1957). Fagen, R. E., Random walks with restraining barrier as applied to the biased binary counter, J. Soc. Indust. Appl. Math. 6, 1 (1958).

M 20, 226

Feller, W., The numbers of zeros and of changes of sign in a symmetric random walk, Enseignement Math. (2) 3, 229 (1957).

M 20, 718

- Fisher, M. E., (See C. Domb) Proc. Cambridge Philos. Soc. 54, 48 (1958).

Flatto, L., A problem on random walk, Quart. $J$. Math. Oxford Ser. (2) 9, 299 (1958). M 20,916

- Foster, C., The case of the forgetful burglar, Amer. Math. Monthlu 65. 71 (1958). M 20, 917 Fröberg, C., Proportional representation systems, Nordisk Mat. Tidskr. 5, 91 (1957). M 19, 692

Fürst, D., La rovina dei giocatori nel caso di riserva limitata, Giorn, Ist. Ital. Attuari 19, 63 (1956). 
Fürst, D., Il caso limite del problema della rovina dei giocatori nell'ipotesi di riserva limitata, Giorn. Ist. Ital. Attuari 20, 120 (1957).

M $\mathbf{2 0}, 942$

Gani, J., Problems, in the probability theory of storage systems, J. Roy. Statist., Ser. B. 19, 181 (1957).

M 19, 1092

Gani, J., Elementary methods for an occupancy problem of storage, Math Ann. 136, 454 (1958).

M 20, 1206

Gillette, D., Stochastic games with zero stop probabilities, Ann Math. Studies, 39, (Princeton University Press, Princeton, N.J., 1957).

M 19, 1147

Gillis, J., Centrally biased discrete random walk, Quart. J. Math. Oxford Ser. \%, 144 (1956). M Ro, 464

Good, I. J., Legendre polynomials and trinomial random walks, Proc. Cambridge Philos. Soc. 54, 39 (1958).

M 21, 442

Good, I. J., Random motion and analytic continued fractions. Proc. Cambridge Philos, Soc. 54, 43 (1958).

M 21, 443

Gower, J. C., (See P. H. Leslie) Biometrika 45, 316 (1958).

Gupta, H. C., Diffusion by continuous movements, J. Math. Phys. 38, 36 (1959/60).

M 21, 318

- Hammersley, J. M., (See S. R. Broadbent) Proc. Cambridge Philos. Soc. 53, 629 (1957).

Hammersley, J. M., Percolation processes. II. The connective constant, Proc. Cambridge Philos. Soc. 53, 642 (1957).

M 19, 989

Hammersley, J. M., Percolation processes: Lower bounds for the critical probability, Ann. Math. Statist. 28, 790 (1957)

M $\mathbf{2 1}, 72$

Hammersley, J. M., Bornes supérieures de la probabilité critique dans un processus de filtration, Colloq. Internat., Paris, Juillet 1958, pp. 17-37. (Centre Nat. de la Recherche Scientifique, Paris, 1959).

M 21, 831

- Haňs, O., (See M. Driml) C.R. Acad. Sci. Paris 248, 629 (1959).

- Hayashi, C., (See S. Sakino) Ann. Inst. Statist. Math., Tokyo 10, 261 (1959).

Henze, E., Beiträge zum Irrfahrtproblem, (Thesis, Technische Hochschule Stuttgart, 1958). M 21, 178

Hida, T., On the uniform continuity of Wiener process with a multidimensional parameter, Nagoya Math. J. 13, 53 (1958).

M 20, 465

Ionescu Tulcea, C., On a class of operators occurring in the theory of chains of infinite order, Canad. J. Math. 11, 112 (1959).

M 21, 73

- Isii, K., (See Y. Taga) Ann. Inst. Statist. Math., Tokyo 11, 25 (1959).

Jackson, J. R., Networks of waiting lines, Operations Res. 5, 518 (1957).

M 19, 1203

Jiřina, M., Stochastic branching processes with continuous state space, Czechoslovak Math. J. 8, (83), 292 (1958).

M 21, 69 $\checkmark$ Jogdeo, S. S., Estimation of most economic number of repairmen required for a set of machines, Proc. Sec. Cong. on Theoretical and Applied Mechanics, New Delhi, October, 1956, pp. 237-252. (Indian Inst. of Tech., Kharagpur.)

M 19, 1092

John, P. W. M., Divergent time homogeneous birth and death processes, Ann Math. Statist. 28, 514 (1957).

M 19, 587

Kac, M., Some remarks on stable processes, Publ. Inst. Statist. Univ. Paris 6, 303 (1957).

M 20, 1019

Kac, M., Remark on recurrence times, Phys. Rev. (2) 115, 1 (1959).

M $\mathbf{2 1}, 1239$

- Kakutani, S., (See A. Dvoretzky) Proc. Cambridge Philos. Soc. 53, 856 (1957).

- Kalaba, R., (See R. Bellman) Proc. Nat. Acad. Sci. U.S.A. 43, 930 (1957).

Kampé de Fériet, J., Fonctions harmoniques aléatoires dans le cercle-unité, C.R. 80éme Congrès des Sociétés Savantes, Lille, Juin 1955, pp. 411-415. (Gauthier-Villars, Paris, 1955.) M 20, 917

- Kampé de Fériet, J., (See G. Birkhoff) J. Math. Mech., 663 (1958).

- Karlin, S., The differential equations of birth-anddeath processes, and the Stieltjes moment problem, Trans. Amer. Math. Soc. 85, 489 (1957).

M 19, 989

- Karlin, S., Many server queueing processes with Poisson input and exponential service times, Pacific J. Math. 8, 87 (1958). $\quad$ M 20, 595

- Karlin, S., Linear growth birth and death processes, J. Math. Mech. \%, 643 (1958). M 20, 812

- Karlin, S., A characterization of birth and death processes, Proc. Nat. Acad. Sci. U.S.A. 45, 375 (1959).

M 21, 177

- Karlin, S., Random walks, Illinois J. Math. 3, 66 (1959).

M $\mathbf{2 0}, 1205$

Kemperman, J. H. B., Asymptotic expansions for the Smirnov test and for the range of cumulative sums, Ann. Math. Statist. 30, 448 (1959). M 21, 320

- Kendall, D. G., The calculation of the ergodic projection for Markov chains and processes with a countable infinity of states, Acta Math. 97, 103 (1957).

M 19, 469

Kendall, D. G., Some problems in theory of dams, J. Roy. Statist. Soc., Ser. B 19, 207 (1957).

M 19, 1092

Kendall, D. G., La propagation d'une épidémie ou d'un bruit dans une population limitée, Publ. Inst. Statist. Univ. Paris 6, 307 (1957). $\quad$ M 20, 1107

Khintchine, A., Nachwirkungsfreie Folgen von zufälligen Ereignissen, Teor. Veroyatnost. i Primenen 1, 3 (1956).

M 19, 328

Khintchine, A., Über Poissonsche Folgen zufälliger Ereignisse, Teor. Veroyatnost. i Primenen 1, 320 (1956).

M 19, 328

van Klinken, J., On some simple stochastic processes of special use in actuarial statistics, VerzekeringsArch. Actuar. Bijv. 35, 107 (1958). $\quad$ M 20, 942

Koronkevič, O. I., Ergodic properties of random functions in the solution of a linear dynamic system, Dopovidi Akad. Nauk Ukrain. RSR 1958, 810 (1958).
M 20, 1107 
amens, A., Sur le processus non homogène de naissance et de mort à deux variables aléatoires, Acad. Roy. Belg. Bull. Cl. Sci. (5) 43, 711 (1957).

M 20, 61

Lamens, A., Sur le processus non homogène de naissance et de mort, Acad. Roy. Belg. Bull. Cl. Sci. (5) 43, 597 (1957).

M 19, 1203

Lamperti, J., An occupation time theorem for a class of stochastic processes, Trans. Amer. Math. Soc. 88, 380 (1958).

M 20, 227

Langebartel, R. G., Average initial velocity of the terminus of a two-dimensional linkage, Proc. Amer. Math. Soc. 10, 128 (1959).

M 21, 727

- Lehman, R. S., A study of the restricted random walk, J. Soc. Indust. Appl. Math. 6, 257 (1958).

M 20, 812

- Lehrer, T. A., (See R. E. Fagen) J. Soc. Indust. Appl. Math. 6, 1 (1958).

Leslie, P. H., A stochastic model for studying the properties of certain biological systems by numerical methods, Biometrika 45, 16 (1958).

M 19, 1245

- Leslie, P. H., The properties of a stochastic model for two competing species, Biometrika 45, 316 (1958)

M 21, 228

Levinson, N., Limiting theorems for Galton-Watson branching process, Illinois J. Math. 3, 554 (1959).

M 21, 1239

Lévy, P., A special problem of Brownian motion, and a general theory of Gaussian random functions, Proc. Third Berkeley Symp. on Math. Stat. and Prob. II, pp. 133-175. (University of Calif. Press, Los Angeles, 1956.) M 19, 893

Lévy, P., Brownian motion depending on $n$ parameters: the particular case $n=5$, Proc. Symp. in Appl. Math. \%, 1. (McGraw-Hill Book Co., New York-Toronto-London, 1957.)

M 19, 893

- Mack, C., The efficiency of $N$ machines unidirectionally patrolled by one operative when walking time and repair times are constants, $J$. Roy. Statist. Soc., Ser. B 19, 166 (1957).

M 19, 1092

Mack, C., The efficiency of $N$ machines uni-directionally patrolled by one operative when walking time is constant and repair times are variable, $J$. Roy. Statist. Soc., Ser. B. 19, 173 (1957). M 19, 1092

Malécot, G., Sur quelques processus de "mouvement brownien", Ann. Univ. Lyon. Sect. A (3) 20, 33 (1957).

M 20, 1019

Marchand, H., Essai d'étude mathématique d'une forme d'épidémie, Ann. Univ. Lyon. Sect. A (3) 19, 13 (1956).

M 19, 932

- Mathews, P. M., Stochastic processes associated with integrals of a class of random functions, Proc. Nat. Inst. Sci. India. Part A 22, 369 (1956).

M 19, 587

McFadden, J. A., The axis-crossing intervals of random functions, IRE Trans.IT-2, 146 (1956).

M 20, 812

McFadden, J. A., The axis-crossing intervals of random functions. II., IRE Trans. IT-4, 14 (1958).
- McGregor, J. L., (See S. Karlin) Trans. Amer. Math. Soc. 85, 489 (1957).

- McGregor, J., (See S. Karlin) Pacific J. Math. 8, 87 (1958).

- McGregor, J., (See S. Karlin) J. Math. Mech. y, 643 (1958).

- McGregor, J., (See S. Karlin) Proc. Nat. Acad. Sci. U.S.A., 45, 375 (1959).

- McGregor, J., (See S. Karlin) Illinois J. Math. 3, 66 (1959).

- McMillan, B., A moving single server problem, Ann. Math. Statist. 28, 471 (1957).

M 19, 514

Meisling, 'T., Discrete-time queuing theory, Operations Res. 6, 96 (1958).

M 19, 1092

- Mercer, A., A random walk in which the steps occur randomly in time, Biometrika 46, 30 (1959).

M 21, 179

- Miller, K. S., Rayleigh processes, Quart. Appl. Math. 16, 137 (1958).

M 20, 227

Mogyoródi, J., Probabilistic treatment of the motion of neutrons in nuclear reactors, Magyar Tud. Akad. Mat. Kutató Int. Közl. 3, 237 (1958).

M 21,849

Montroll, E. W., Random walks in multidimensional spaces, especially on periodic lattices, J. Soc. Indust. Appl. Math. 4, 241 (1956).

M 19, 470

Motoo, M., Some theorems on the sum of positive random variables, Ann. Inst. Statist. Math., Tokyo \%, 169 (1956).

M 19, 468

Motoo, M., Proof of the law of iterated logarithm through diffusion equation, Ann. Inst. Statist. Math. 10, 21 (1958).

M 20, 718

- Motoo, M., (See H. Watanabe) J. Math. Soc. Japan 10, 272 (1958).

- Mott, J. L., Matrix norms applied to weakly ergodic Markov chains, Arch. Math. 8, 331 (1957).

M 19, 988

Muller, M. E., Some continuous Monte Carlo methods for the Dirichlet problem, Ann. Math. Statist. 27, 569 (1956).

M 19, 580

- Murphy, T., (See C. Mack) J. Roy. Statist. Soc., Ser. B 19, 166 (1957).

- Mycielski, J., Sur un problemé du calcul de probabilité. I. Le mouvement d'une molécule sur une droite, Studia Math. 15, 188 (1956).

M 19, 588

Nedoma, J., The capacity of a discrete channel, Trans. 1st. Prague Conf. on Information Theory, Liblice, Nov. 1956, pp. 143-181. (Czech. Acad. of Sciences, Prague, 1957.)

M $\mathbf{2 1}, 229$

O'Connor, D., A simplified method of solution for a random walk problem of a few unequal steps, Acta Phys. Polon. 17, 273 (1958).

M 20, 595

Paszkowski, S., (See J. Mycielski) Studia Math. 15, 188 (1956).

Petrov, V. V., A local theorem for latticed distributions, Dokl. Akad. Nauk SSSR (N.S.), 115, 49 (1957)

M 20, 58

Prabhu, N. U., On the integral equation for the finite dam, Quart. J. Math. Oxford, Ser. (2) 9, 183 (1958).

M 20, 1020 
Pyke, R., On renewal processes related to type I and type II counter models, Ann. Math. Statist. 29, 737 (1958).

M 20, 917

Pyke, R., The supremum and infimum of the Poisson process, Ann. Math. Statist. 30, 568 (1959).

M 21, 1125

- Rapport, A., (See C. Foster) Amer. Math. Monthly 65, 71 (1958).

Reich, E., Waiting times when queues are in tandem, Ann. Math. Statist. 28, 768 (1957).

M 19, 1203

Rényi, A., A characterization of Poisson processes, Magyar Tud. Akad. Mat. Kutató Int. Közl. 1, 519 (1956).

M 20, 227

Reuter, G. E. H., (See D. G. Kendall) Acta Math. 9\%, 103 (1957).

- Riordan, J., (See B. MeMillan) Ann. Math. Statist. 28, 471 (1957).

Roby, N., Sur certains processus remarquables généralisant les processus de Poisson, C.R.Acad. Sci. Paris 248, 2945 (1959).

M 21, 443

Rosenblatt, M., A multi-dimensional prediction problem, Ark. Mat. 3, 407 (1958). M 19, 1098

Sack, R. A., Restricted random walks and the use of moments, Phil. Mag. (8) 3, 504 (1958). M 20, 59

Sacks, J., Asymptotic distribution of stochastic approximation procedures, Ann. Math. Statist. 29, 373 (1958).

M 20, 811

- Sakino, S., On the analysis of epidemic model. I. (Theoretical approach), Ann. Inst. Statist. Math., Tokyo. 10, 261 (1959).

M 21, 831

- Scheerer, A., (See B. Epstein) J. Analyse Math. 4, $222(1955 / 56)$.

Schneider, B., Stochastische Grundlagen für eine statistische Auswertung der EEG-Kurven, Mitt. Math. Sem. Giessen 56, 49 pp. (1958). M $\mathbf{2 0 ,} 124$

- Schneider, H., (See J. L. Mott) Arch. Math. 8, 331 (1957).

Schwartz, L., La fonction aléatoire du mouvement Brownien, Mimeographed paper from Séminaire Bourbaki 195\%-8. (Secrétariat mathématique, Paris, 1958.)

M 21, 1125

Senju, S., The optimal assignment of operatives in serving automatic machines, Proc. Fac. Engrg. Keio Univ. 9, 81 (1956).

M 19, 1091

Sevast'yanov, B. A., Branching stochastic processes for particles diffusing in a bounded domain with abosrbing boundaries, Teor. Veroyatnost. i Primenen. 3, 121 (1958).

M 20, 718

Sevast'yanov, B. A., Transient phenomena in branching stochastic processes, Teor. Veroyatnost. i Primenen. 4, 121 (1959).

M 21, 726

Sherman, B., The limiting distribution of Brownian motion in a bounded region with instantaneous return, Ann. Math. Statist. 99, 267 (1958).

M 20, 60

- Siegel, A., (See N. Wiener) Nuovo Cimento (10) 2 , 982 (1955).

- Siegel, A., "Theory of measurement" in differential-space quantum theory, Phys. Rev. (2) 101, 429 (1956).

M 20, 109
Sjöberg, B., Über lineare Irrfahrt mit Absorptionsschranken, Acta Acad. Abo. 21, no. 13, 13 pp. (1958).

M $\mathbf{2 1}, 72$

Sjöberg, B., Über Brownsche Bewegung mit Absorptionsschranken, Acta Acad. Abo. 21, no. 14, 12 pp. (1959).

M 21, 977

- Smith, C. S., (See A. Mercer) Biometrika 46, no. $1 / 2,30(1959)$.

Smith, W. L., Renewal theory and its ramifications, J. Roy. Statist. Soc., Ser. B 20, 243 (1958).

M $\mathbf{2 0}, 918$

Spitzer, F., Some theorems concerning 2-dimensional Brownian motion, Trans. Amer. Math. Soc. 8\%, 187 (1958).

M 21,579

- Srinivasan, S. K., (See P. M. Mathews) Proc. Nat. Inst. Sci. India. Part A $\mathbf{2 2}, 369$ (1956).

Stephan, F. F., Two queues under preemptive priority with Poisson arrival and service rates, Operations Res. 6, 399 (1958).

M 19, 1203

Taga, Y., On a stochastic model concerning the pattern of communication: Diffusion of news in a social group, Ann. Inst. Statist. Math. Tokyo 11, $25(1959)$

M 21, 1019

Takács, L., On a stochastic process concerning some waiting time problems, Teor. Veroyatnost. $i$ Primenen. 2, 92 (1957).

M 19, 692

Takács, L., On secondary stochastic processes generated by a multidimensional Poisson process, Magyar Tud. Akad. Mat. Kutató Int. Közl. 刃, 71 (1957).

M 20, 1205

Takács, L., On random walk problems, Magyar Tud. Akad. Mat. Kutató Int. Közl. 2, 81 (1957).

M $\mathbf{2 0}, 917$

Takács, L., Remarks on random walk problems, Magyar Tud. Akad. Mat. Kutató Int. Közl. 2, 175 (1957).

M 21,179

Taylor, S. J., (See A. Dvoretzky) Proc. Cambridge Philos. Soc. 53, 856 (1957).

Theodorescu, R., Sur certains processus à liaisons complètes, Atìi Accad. Naz. Lincei. Rend. Cl. Sci. Fis. Mat. Nat. (8) $\mathbf{2 4}, 260$ (1958). $\quad$ M $\mathbf{2 0}, 718$

Tortrat, A., Itération de certaines matrices et processus de Markoff, C.R. Acad. Sci. Paris \$45, 1872 (1957).

M 19, 1093

Tortrat, A., Etude d'une méthode d'itération propre à certaines matrices, application aux processus de Markoff correspondants, cas des processus continus homogènes par rapport à l'espace, cas non homogène, Publ. Sci. Univ. Alger., Sér. A 4, 145 (1957).

M 21, 71

Tortrat, A., (See A. Blanc-Lapierre) (Masson et Cie, Paris, 180 pp., 1959).

Trotter, H. F., A property of Brownian motion paths, Illinois J. Math. 2, 425 (1958).

M $\mathbf{2 0}, 464$

Trotter, H. F., Approximation of semi-groups of operators, Pacific J. Math. 8, 887 (1958).

M $\mathbf{2 1}, 422$

Urbanik, K., On a stochastic model of a cascade, Studia Math. 16, 237 (1958). M 20,61

Urbanik, K., An effective example of a Gaussian function, Bull. Acad. Polon. Sci. Sér. Sci. Math. Astr. Phys. 7, 343 (1959). 
- Varga, R. S., (See G. Birkhoff) J. Soc. Indust. Appl. Math. 6, 354 (1958).

Ventcel, A. D., On lateral conditions for multidimensional diffusion processes, Teor. Veroyatnost. $i$ Primenen. 4, 172 (1959).

M $\boldsymbol{2 1}, 978$

- Watanabe, H., Ergodic property of recurrent diffusion processes, J. Math. Soc. Japan 10, 272 (1958).

M 21,73

Watanabe, Y., Berichtigung zu meiner früheren Note "Aufgaben betreffend das Irrfahrtproblem", $J$. Gakugei Tokushima Univ. Nat. Sci. Math. ', 36 (1955).

M 19, 692

Watanabe, Y., Einige Erweiterung des Pólyaschen Irrfahrtproblem, J. Gakugei Tokushima Univ. 8, 13 (1957).

M 20, 338

- Webb, N. L., (See C. Mack) J. Roy. Statist. Soc., Ser. B 19, 166 (1957).

- Weiss, G. H., (See R. S. Lehman) J. Soc. Indust. Appl. Math. 6, 257 (1958).

Wendel, J. G., Groups and conditional Monte Carlo, Ann. Math. Statist. 28, 1048 (1957). $\quad$ M 20, 68

- White, H., Queuing with preemptive priorities or with breakdown, Operations Res. 6, 79 (1958).

M 19, 1091

- Wiener, N., The differential-space theory of quantum systems, Nuovo Cimento (10) 2, 982 (1955).

M 20, 109

- Wiener, N., (See A. Siegel) Phys. Rev. (2) 101, 429 (1956).

- Wiener, N., The definition and ergodic properties of the stochastic adjoint of a unitary transformation, Rend. Circ. Mat. Palermo (2) 6, 205 (1957).

M 20, 718

Zitek, F., On a theorem of Korolyuk, Czechoslovak Math. J. g (82), 318 (1957).

M 19, 778

Zitek, F., Zur Theorie der gemischten Wartesysteme, Apl. Mat. 2, 154 (1957).

M 19, 692

Zitek, F., Zur 'Theorie der ordinären nachwirkungsfreien Folgen, Czechoslovak Math. J. 8 (83), 448 (1958).

M 21, 72

Zolotarev, V. M., More exact statements of several theorems in the theory of branching processes, Teor. Veroyatnost. i Primenen. 2, 256 (1957).

M 20, 465

\section{E. Applications}

Adhikari, B. P., Distance, discrimination et résumé exhaustif, Publ. Inst. Statist. Univ. Paris 5, 57 (1956).

M 19, 329

Akaike, H., On ergodic property of a tandem type queueing process, Ann. Inst. Statist. Math., Tokyo 9, 13 (1957).

M 19, 990

Alkemade, C. T. J., On the problem of Brownian motion of non-linear systems, Physica 24, 1029 (1958).

M 21, 330

Amara, R. C., Application of matrix methods to the linear least squares synthesis of multivariable systems, J. Franklin Inst. 268, 1 (1959). M $\mathbf{2 1 ,} 890$

- Arató, M., Probabilistic proof of a theorem on the approximation of continuous functions by means of generalized Bernstein polynomials, Acta Math. Acad. Sci. Hungar. 8, 91 (1957).

M 19, 411
Barrer, D. Y., Queuing with impatient customers and indifferent clerks, Operations Res. 5, 644 (1957).

M 19, 779

Barrer, D. Y., Queuing with impatient customers and ordered service, Operations Res. 5, 650 (1957).

M 19, 779

Bašarin, G. P., Final probabilities for multi-dimensional Markov processes which describe the action of some two-stage telephone busy-signal systems, Teor. Veroyatnost. i Primenen. 3, 452 (1958).

M 21,179

Basharin, G. P., An investigation, using probability theory, of a two-stage trunk-hunting telephone system with refused calls, Soviet Physics. Dokl. 121 (3), 713 (1958).

M 21, 72

- Bellman, R., On the principle of invariant imbedding and one-dimensional neutron multiplication, Proc. Nat. Acad. Sci. U.S.A. 43, 517 (1957).

M 19, 506

Bendat, J. S., Principles and applications of random noise theory (John Wiley \& Sons, Inc., New York, 1958).

M $\mathbf{2 1}, 832$

Beneš, V. E., On queues with Poisson arrivals, Ann. Math. Statist. 28, 670 (1957).

M 19, 990

Beneš, V. E., On trunks with negative exponential holding times serving a renewal process, Bell System Tech. J. 38, 211 (1959).

M 21,72

Bennett, W. R., Statistics of regenerative digital transmission, Bell System Tech. J. 3\%, 1501 (1958).

M 21, 179

Beran, M. J., Determination of the intensity distribution resulting from the random illumination of a plane finite surface, Opt. Acta 5, 88 (1958).

M 21, 1001

Béthoux, P., Nombre maximum de signaux d'énergie totale fixée parmi lesquels on peut discriminer à $\epsilon$ près en présence d'un bruit blanc Gaussien, $C . R$. Acad. Sci. Paris 24\%, 573 (1958).

M 20, 1136

Blachman, N. M., Limiting frequency-modulation spectra, Information and Control 1, 26 (1957).

M 19, 1093

Blachman, N. M., On Fourier series for Gaussian noise, Information and Control 1, 56 (1957).

M 19, 1093

- Blachman, N., Optimum search for objects having unknown arrival times, Operations Res. $\%, 625$ (1959).

M 21, 1408

Blackman, R. B., The measurement of power spectra from the point of view of communications engineering. I., Bell System Tech. J. 3\%, 185 (1958).

M 21,323

- Blackman, R. B., The measurement of power spectra from the point of view of communications engineering. II., Bell System Tech. J. 3\%, 485 (1958).

M $\mathbf{2 1}, 323$

Blum, M., On exponential digital filters, J. Assoc. Comput. Mach. 6, 283 (1959).

M 21, 362

Boguslavskiı̌, I. A., On a class of nonstationary random process filtering, Avtomat. $i$ Telemeh. 20, 708 (1959).
M 21, 890 
Bolie, V. W., Theory of scattering from a nearly transparent anomaly, Appl. Sci. Res. B. 6, 422 (1957).

M 19, 709

- Broadbent, S. R., Percolation processes. I. Crystals and mazes, Proc. Cambridge Philos. Soc. 53, 629 (1957).

M 19, 989

Buch, K. R., On a special use of the Erlang methods in industry, Teleteknik 1, 76 (1957). M 19, 470

Bunimovich, V. I., Fluctuating processes as oscillations with random amplitudes and phases, Akad. Nauk SSSR Zurnal Tehn. Fiz. 19, 1231 (1949). (Translated by Morris D. Friedman, 572 California St., Newtonville 60, Mass., 1956.) M 19, 360

Burke, P. J., Equilibrium delay distribution for one channel with constant holding time, Poisson input and random service, Bell System Tech. J. 38, 1021 (1959).

M 21, 1125

Canceill, B. (See R. Fortet), Teleteknik 1, 41 (1957). Caughey, T. K., Response of a nonlinear string to random loading, J. Appl. Mech. 26, 341 (1959).

M 21, 1140

Caughey, T. K., Response of Van der Pol's oscillator to random excitation, J. Appl. Mech. 26, 345 (1959).

M 21, 1239

Chernoff, H., The distribution of shadows, $J$. Math. Mech. 6, 567 (1957).

M 19, 890

Clarke, A. B., Maximum likelihood estimates in a simple queue, Ann. Math. Statist. 28, 1036 (1957).

M 20, 60

Cohen, J. W., Certain delay problems for a full availability trunk group loaded by two traffic sources, Communication News 16, 105 (1956). M 19, 235

Cohen, J. W., The full availability group of trunks with an arbitrary distribution of the inter-arrival times and a negative exponential holding time distribution, Simon Stevin 31, 169 (1957).

M 20, 917

Conolly, B. W., A difference equation technique applied to the simple queue, J. Roy. Statist. Soc., Ser. B 20, 165 (1958).

M $\mathbf{2 0}, 464$

Conolly, B. W., A difference equation technique applied to the simple queue with arbitrary arrival interval distribution, J. Roy. Statist. Soc. Ser. B 20, 168 (1958).

M 20, 464

Conolly, B. W., The busy period in relation to the queueing process $\mathrm{GI} / \mathrm{M} / 1$, Biometrika 46, $1 / 2,246$ (1959).

M 21, 317

Crandall, S. H., Notes for the M.I.T. special summer program on random vibration (Technology Press, Massachusetts Institute of Technology, Cambridge, 1958).

M $\mathbf{2 1}, 456$

Culbertson, J. T., Mathematics and logic for digital devices (D. Van Nostrand Co., Inc., Princeton, N.J., 1958).

M 19, 1200

Daly, J. F., (See H. Chernoff) J. Math. Mech. 6, 567 (1957).

Darlington, S., Linear least-squares smoothing and prediction, with applications, Bell System Tech. J. 3\%, 1221 (1958).

M 21, 229

Davis, R. C., Optimum vs. correlation methods in tracking random signals in background noise, Quart. Appl. Math. 15, 123 (1957). M 20, 1208
Delange, H., Sur les fonctions arithmétiques fortement additives, C.R. Acad. Sci. Paris $\mathbf{2 4 4 ,} 1307$ (1957).

M 19, 394

Delange, H., Sur les fonctions arithmétiques fortement additives, C.R. Acad. Sci. Paris 244, 1604 (1957).

M 19, 394

Delange, H., Sur les fonctions arithmétiques fortement additives, C.R. Acad. Sci. Paris 244, 2122 (1957).

M 19, 394

Downton, F., A note on Moran's theory of dams, Quart. J. Math. Oxford Ser. (2) 8, 282 (1957).

M 21, 74

Dyer, I., Response of plates to a decaying and convecting random pressure field, J. Acoust. Soc. Amer. 31, 922 (1959).

M 21, 1139

Elias, P., Error-free coding, Trans. I.R.E. PGIT-4, 29 (1954).

M 19, 721

Elldin, A., Brief presentation of the theory of telephone traffic, Ericsson Rev. 25, 13 (1958).

M 19, 1093

Erdös, P., On additive arithmetical functions and applications of probability to number theory, Proc. Int. Cong. Math. 3, 13. (North-Holland Publ. Co., Amsterdam, 1956.) M 19, 393

- Erdös, P., Some further statistical properties of the digits in Cantor's series, Acta Math. Acad. Sci. Hungar. 10, 21 (1959). $\quad$ M 21,1179

Eringen, A. C., Response of beams and plates to random loads, J. Appl. Mech. 24, 46 (1957).

M 19, 340

- Eringen, A. C., (See J. C. Samuels) J. Appl. Mech. 25, 496 (1958).

- Eringen, A. C., (See J. C. Samuels) J. Math. Phys. 38, 83 (1959/60).

Erohin, V., $\epsilon$-entropy of a discrete random variable, Teor. Veroyatnost. i Primenen. 3, 103 (1958).

M 20, 126

Faure, P., Sur quelques résultats relatifs aux fonctions aléatoires stationnaires isotropes introduites dans l'étude expérimentale de certains phénomènes de fluctuations, C.R. Acad. Sci. Paris 244, 842 (1957).

M 19, 72

- Faure, P., Etude théorique de la variation, en fonction de l'aire de mesure, du coefficient de Selwyn, défini à propos de la granularité des films photographiques, C.R. Acad. Sci. Paris $\mathbf{2 4 4}$, 2371 (1957).

M 19, 1009

Feinstein, A., Foundations of information theory, (McGraw-Hill Book Co., Inc., N.Y., 1958).

M $\mathbf{2 0}, 262$

Finch, P. D., The effect of the size of the waiting room on a simple queue, J. Roy. Statist. Soc., Ser. $B$ 20, 182 (1958). M 20, 596

Finch, P. D., Balking in the queueing system GI/ M/1, Acta Math. Acad. Sci. Hungar. 10, 241 (1959).

M 21, 579

Finch, P. D., Cyclic queues with feedback, J. Roy. Statist. Soc., Ser. B 21, 153 (1959). M 21, 1237

- Fortet, R., Probabilités de perte en selection conjuguée, Teleteknik 1, 41 (1957). M 19, 587

Freeman, J. J., Principles of noise (John Wiley \& Sons, Inc., New York, 1958). $\quad$ M 20,719 
- Freund, J. E., (See I. Miller) J. Appl. Phys. 2\%, 1290 (1956).

Friedland, B., Least squares filtering and prediction of non-stationary sampled data, Information and Control 1, 297 (1958).

M 21, 73

Fürst, D., Il caso limite del problema della rovina dei giocatori nell'ipotesi di riserva limitata, Giorn. Ist. Ital. Attuari 20, 120 (1957). $\quad$ M 20, 942

• Galliher, H. P., Non-stationary queueing probabilities for landing congestion of aircraft, Operations Res. 6, 264 (1958).

M $\mathbf{2 0}, 60$

- Gani, J., The time-dependent solution for a storage model with Poisson input, J. Math. Mech. 8, 653 (1959).

M 21, 830

- Gani, J., Remarks on the dam with Poisson type inputs, Austral. J. Appl. Sci. 10, 113 (1959).

M 21, 1238

Gaver, D. P., Imbedded Markov chain analysis of a waiting-line process in continuous time, Ann. Math. Statist. 30, 698 (1959).

M 21, 1238

George, F. H., Logical networks and probability, Bull. Math. Biophys. 19, 187 (1957). M 19, 1245

Girault, M., Files d'attente. Loi de survie d'un intervalle à partir d'un instant quelconque, C.R. Acad. Sci. Paris 246, 2838 (1958). M 20, 60

Gnedenko, B. V., On a problem of mass service, Dopovidi Akad. Nauk Ukrain. RSR, 1958, 477 (1958).

M 20, 812

Gnedenko, B. V., On a generalization of Erlang's formulae, Dopovidi Akad. Nauk Ukrain. RSR, 1959, 347 (1959).

M 21, 579

Good, I. J., Could a machine make probability judgments? I., II., Computers and Automation 8, 14 and 24 (1958).

M 21, 84

Grenander, U., On the theory of mortality measurement, II., Skand. Aktuarietidskr. 39, 125 (1957).

M 19, 1243

Grenander, U., On heterogeneity in non-life insurance. I., Skand. aktuarietidskr. 40, 71 (1957).

M 19, 1243

Haight, F. A., Queueing with balking, Biometrika 44, 360 (1957).

M 19, 692

Haight, F. A., Two queues in parallel, Biometrika 45, 401 (1958).

M $\mathbf{2 0}, 1106$

Hammersley, J. M., Percolation processes. II. The connective constant, Proc. Cambridge Philos. Soc. 53, 642 (1957).

M 19, 989

- Hammersley, J. M., (See S. R. Broadbent) Proc. Cambridge Philos. Soc. 53, 629 (1957).

Harris, T. E., The random functions of cosmic-ray cascades, Proc. Nat. Acad. Sci. U.S.A. 43, 509 (1957).

M 19, 328

Harrison, G., Stationary single-server queueing processes with a finite number of sources, Operations Res. $\boldsymbol{~ \% , ~} 458$ (1959).

M 21,978

- Hazelwood, R. N., (See L. G. Peck) Publications in Operations Research, No. 2. (John Wiley \& Sons, Inc., N.Y., 1958.)

Heathcote, C. R., The time-dependent problem for a queue with preemptive priorities, Operations Res. ช, $670(1959)$.
Hilibrand, J., Characterization of probability distributions for excess physical noises, Tech. Rep. $\mathbf{2} \boldsymbol{\gamma} 6$ (Mass. Inst. of Tech., Cambridge, Mass., 1956).

M 19, 1203

Hori, J., On the vibration of disordered linear lattice. II., Progr. Theoret. Phys. 18, 367 (1957).

M 19, 1217

Husu, A. P., On some functionals on random fields, Vestnik Leningrad. Univ. 12, no. 1, 37 (1957).

M 19, 468

Hyvärinen, L. P., The autocorrelation and the power spectrum of nonstationary shot noise, Acta Pclytech. Scandinav. 252, 23 pp. (1958).

M $\mathbf{2 1}, 73$

Ivanenko, I. P., On the equilibrium angular distribution function of particles in a cascade shower, Soviet Physics. Dokl. 122 (3), 962 (1958).

М $\mathbf{2 1}, 580$

Jackson, J. R., Multiple servers with limited waiting space, Naval Res. Logist. Quart. 5, 315 (1958).

M $\mathbf{2 0}, 1106$

Jacobsen, B. B., Probability theory in telephone transmission, Teleteknik 1, 83 (1957). M 19, 374

Jánossy, L., The fluctuations of intensity of an extended light source, Nuovo Cimento (10) 12, 369 (1959).

M $\mathbf{2 1}, 1282$

Jensen, E. L., Elementary queue theory, Nordisk Mat. Tidskr. 6, 137 (1958).

M 21, 579

- Joshi, D. D., (See B. P. Adhikari) Publ. Inst. Statist. Univ. Paris 5, 57 (1956).

Kac, M., Probability in classical physics, Proc. Symposia in Appl. Math. \%, pp. 73-85. (McGraw-Hill Book Co., N.Y., 1957).

M 20, 338

- Kalaba, R., (See R. Bellman) Proc. Nat. Acad. Sci. U.S.A. 43, 517 (1957).

Kalecki, M., Mechanistic model of a random phenomenon, Zastos. Mat. 4, 113 (1958). M $\mathbf{2 0}, 917$

Kampé de Fériet, J., Intégrales aléatoires de l'équation de la diffusion, C.R. Acad. Sci. Paris $\mathbf{2 4 3 ,} 929$ (1956).

M 19, 40

Kampé de Fériet, J., Problèmes mathématiques posés par la mécanique statistique de la turbulence, Proc. Int. Cong. Math. 3, 237. (NorthHolland Publ. Co., Amsterdam, 1956.) M 19, 490

Kampé de Fériet, J., Construction de mesures dans certains espaces fonctionnels en vue des applications à la physique mathématique, Séminaire surles problèmes mathématiques de la physique théorique VIII, 22 pp. (Université de Lille, 1956-57.)

M 19, 735

van Kampen, N. G., Thermal fluctuations in a nonlinear system, Phys. Rev. (2) 110, 319 (1958).

M $\mathbf{2 0}, 820$

- Karlin, S., Many server queueing processes with Poisson input and exponential service times, Pacific J. Math. 8, 87 (1958).

M 20,595

- Karlin, S., Note on a moving single server problem, Ann Math. Statist. 30, 243 (1959).

M 21, 72

- Kay, I., Multiple scattering by a random stack of dielectric slabs, Nuovo Cimento (10) 9, 626 (1958).

M $\mathbf{2 1}, 179$ 
- Kesten, H., Priority in waiting line problems. I., Nederl. Akad. Wetensch.Proc., Ser. A. $60=$ Indag. Math. 19, 312 (1957).

M 19, 719

- Kesten, H., Priority in waiting line problems. II., Nederl. Akad. Wetensch. Proc., Ser. A. $\mathbf{6 0}=$ Indag. Math. 19, 325 (1957).

M 19, 719

Khinchin, A. I., Mathematical foundations of information theory (Translated by R. H. Silverman and M. D. Friedman, Dover Publ., N.Y., 1957).

M 19, 1148

Kimura, M., Some problems of stochastic processes in genetics, Ann. Math. Statist. 28, 882 (1957).

M $\mathbf{2 0}, 125$

Kitagawa, T., Some aspects of stochastically approximative analysis, Bull. Math. Statist. 6, 109 (1956).

M 19, 461

van Klinken, J., Some remarks on dependent and independent probabilities, Verzekerings-Arch. Actuarieel Bijoegsel 35, 13 (1958).

M 19, 1145

Kochen, M., On the commutativity of operators in stochastic models for learning, Ann. Math. Statist. 29, 930 (1958).

M $\mathbf{2 0 ,} 813$

Kochen, M., Extension of Moore-Shannon model for relay circuits, IBM J. Res. Develop. 3, 169 (1959).

M 20, 1242

Koopman, B. O., Quantum theory and the foundations of probability, Proc. Symposia in Appl. Math. VII, pp. 97-102. (McGraw-Hill Book Co., N.Y., 1957.)

M 19, 610

Kovalenko, I. M., Determining the correlation functions of some processes associated with serving problems, Dopcvidi Akad. Nauk Ukraïn. RSR 1958, 480 (1958)

M 20, 812

Krasovskiǔ, A. A., On synthesis of impulsive compensation devices of servosystems, Avtomat. $i$ Telemeh. 20, 729 (1959).

M 21, 749

Kurakin, K. I., An analytical method for the design of linear automatic control systems with prescribed dynamic accuracy in the presence of noise, Avtomat. $i$ Telemeh. 19, 408 (1958).

M 20, 619

Lambert, F., Les problèmes d'attente, Cahiers Centre Etudes Rech. Oper. no. 2, 5 (1959).

M 21, 443

- Leonov, Y. P., Estimation of parameters of the probability distribution of a random function with incomplete a priori information, Avtomat. i Telemeh. 16, 985 (1957).

M 20, 918

Litwiniszyn, J., Application of the equation of stochastic processes to mechanics of loose bodies, Arch. Mech. Stos. 8, 393 (1956).

M 19, 599

Litwiniszyn, J., Fundamental principles of the mechanics of stochastic media, Proc. Third Congress on Theor. and Appl. Mechanics, Bangalore, Dec. 1957, pp. 93-110. (Indian Institute of Technology, Kharagpur, 1958.)

M P0, 1220

Litwiniszyn, J., Flows in pipe networks from the point of view of the theory of random processes, Arch. Mech. Stos. 11, 421 (1959).

M 21, 1239

Longuet-Higgins, M. S., On the transformation of a continuous spectrum by refraction, Proc. Cambridge Philos. Soc. 53, 226 (1957).

M 19, 329
Longuet-Higgins, M. S., On the velocities of the maxima in a moving wave-form, Proc. Cambridge Philos. Soc. 53, 230 (1957).

M 19, 329

Longuet-Higgins, M. S., The statistical analysis of a random, moving surface, Philos. Trans. Roy. Soc. London, Ser. A $\mathbf{2 4 9 ,} 321$ (1957).

M 19, 328

Longuet-Higgins, M. S., Statistical properties of an isotropic random surface, Philos. Trans. Roy. Soc. London, Ser. A 250, 157 (1957). $\quad$ M 19, 989

Longuet-Higgins, M. S., The statistical distribution of the curvature of a random Gaussian surface, Proc. Cambridge Philos. Soc. 54, 439 (1958).

M $\mathbf{2 0}, 338$

Longuet-Higgins, M. S., The distribution of the sizes of images reflected in a random surface, Proc. Cambridge Philos. Soc. 55, 91 (1959). M 20, 918 Luchak, G., The distribution of the time required to reduce to some preassigned level a single-channel queue characterized by a time-dependent Poissondistributed arrival rate and a general class of holding times, Operations Res. 5, 205 (1957).

M 19, 514

Luchak, G., The continuous time solution of the equations of the single channel queue with a general class of service-time distributions by the method of generating functions, J. Roy. Statist. Soc., Ser. B \%o, 176 (1958).

M $\mathbf{2 0}, 464$

McGregor, J., (See S. Karlin) Pacific J. Math. 8, 87 (1958).

- Middleton, D., Detection and extraction of signals in noise from the point of view of statistical decision theory. II., J. Soc. Indust. Appl. Math. 4, 86 (1956).

M 19, 824

- Middleton, D., (See J. S. Mullen) Quart. Appl. Math. 15, 395 (1958).

- Miller, I., Some results on the analysis of random signals by means of a cut-counting process, J. Appl. Phys. 2\%, 1290 (1956).

M 19, 72

- Miller, R. G., (See S. Karlin) Ann. Math. Statist. 30, 243 (1959).

Montroll, E. W., The application of the theory of stochastic processes to chemical kinetics, Advances in Chemical Physics 1, pp. 361-399. (Interscience Publishers, New York and London,1958.)

M $\mathbf{2 1}, 330$

Moran, P.A.P., A probability theory of a dam with a continuous release, Quart. J. Math. Oxford Ser. (2) $\boldsymbol{7}, 130(1956)$.

M 21, 73

Moyal, J. E., Theory of the ionization cascade, Nuclear Phys. 1, 180 (1956). M 20,596

- Mullen, J. A., The rectification of non-Gaussian noise, Quart. Appl. Math. 15, 395 (1958).

M 19, 894

Münch, G., Stochastic processes of astronomical interest, Proc. Symposia in Appl. Math. VII, pp. 51-66. (McGraw-Hill Book Co., N.Y., 1957.)

M 19, 779

Mycielski, J., Sur un problème du calcul de probabilité. I. Le mouvement d'une molécule sur une droite, Studia Math. 15, 188 (1956).

M 19, 588 
Nelson, R. T., Waiting-time distributions for application to a series of service centers, Operations Res. 6, 856 (1958).

M 20, 1019

- Neyman, J., Stochastic models of population dynamics, Science 130, 303 (1959).

M 21, 1164

Parasiouk, O. S., Sur le problème de la filtration des processus stationnaires généralisés, Ukrain. Mat. Ž. 9, 210 (1957).

M 19, 892

Peck, L. G., Finite queuing tables, Publications on Operations Research No. 2. (John Wiley and Sons, Inc., N.Y., 1958).

M 19, 1146

Phillips, O. M., The equilibrium range in the spectrum of wind-generated waves, J. Fluid Mech. 4, 426 (1958)

M 20, 87

Pierson, W. J., On the phases of the motions of ships in confused seas, College of Engineering'Tech. Report 9, Nonr 285. (New York University, N.Y., 1957.)

M 19, 1241

Pollack, M., Some studies on shuttle and assemblyline processes, Naral Res. Logist. Quart. 5, 125 (1958).

M $\mathbf{2 0}, 60$

Pollaczek, F., Détermination de différentes fonctions de répartition relatives à un groupe de lignes téléphoniques sans dispositif d'attente, C.R. Acad. Sci.Paris $\mathbf{9 4}, 1826$ (1958).

M 20, 1205

Pollaczek, F., Fonctions de répartition relatives à un groupe de lignes téléphoniques sans dispositif d'attente, C. R. Acad. Sci. Paris 248, 353 (1959).

M 20, 1205

Postnikov, A. G., Additive problems with a growing number of terms Izv. Akad. Nauk SSSR. Ser. Mat. 20, 751 (1956).

M 19, 252

Prabhu, N. U., Some exact results for the finite dam, Ann. Math. Statist. 29, 1234 (1958). M 20, 1019

- Prabhu, N. U., (See S. Karlin) Ann. Math. Statist. 30, 243 (1959).

- Prabhu, N. U., (See J. Gani) J. Math. Mech. 8, 653 (1959).

- Prabhu, N. U., (See J. Gani) Austral. J. Appl. Sci. 10, $113(1959)$.

Prabhu, N. U., Application of generating functions to a problem in finite dam theory, $J$. Austral. Math. Soc. 1, part 1, $116(1959 / 61)$. M 21, 831

Pratt, J. W., Admissible one-sided tests for the mean of a rectangular distribution, Ann. Math. Statist. 99, 1268 (1958).

M 20, 1109

Price, R., The detection of signals perturbed by scatter and noise, Trans. I.R.E. PGIT-4, 163 (1954).

M 19, 587

- Proschan, F., (See N. Blachman) Operations Res. \%, $625(1959)$.

Pugačev, V. S., Theory of random functions and its application to problems of automatic control, (Gosudarstv. Izdat. Tehn.-Teor. Lit., Moscow, 659 pp., 1957).

M 20, 61

Pugačev, V. S., Determination of optimal system in terms of a general criterion, Avtomat. $i$ Telemeh. 19, 519 (1958).

M $\mathbf{2 0}, 619$

- Ramakrishnan, A., On the distribution of visible stars, Astrophys. J. 126, 573 (1957). M 19, 1142

Rapoport, A., Contribution to the theory of random and biased nets, Bull. Math. Biophys. 19, 257 (1957).
Rashevsky, N., Contributions to the theory of imitative behavior, Bull. Math. Biophys. 19, 91 (1957).

M 19, 374

Reed, I. S., On the use of Laguerre polynominals in treating the envelope and phase components of narrowband Gaussian noise, Trans. I.R.E. I'T-5, 102 (1959).

M 21, 1408

Reich, E., On the integrodifferential equation of Takács. I., Ann. Math. Statist. 29, 563 (1958).

M 20, 60

Reich, E., On the integrodifferential equation of Takács. II., Ann. Math. Statist. 30, 143 (1959). M 20, 1205

Rényi, A., (See M. Arató) Acta Math. Acad. Sci. Hungar. 8, 91 (1957).

- Rényi, A., (See P. Erdös) Acta Math. Acad. Sci. Hungar. 10, 21 (1959).

Rice, S. O., Distribution of the duration of fades in radio transmission: Gaussian noise model, Bell System Tech. J. 3\%, 581 (1958).

M $\mathbf{2 0}, 125$

Romaní, J., A model of the theory of queues with a variable number of channels, Trabajos Estadist. 8, 175 (1957).

M 19, 893

Runnenburg, J. Th., Machines served by a patrolling operator, Math. Centrum Amsterdam. Statist. Afdeling Rep. S 221, 13 pp. (1957).

M 19, 587

- Runnenburg, J. Th., (See H. Kesten) Nederl. Akad. Wetensch. Proc. Ser. A. 60-Indag. Math. 19, 312 (1957).

- Runnenburg, J. Th., (See H. Kesten) Nederl. Akad. Wetensch. Proc. Ser. A. 60-Indag. Math. 19, 325 (1957).

Saaty, T. L., Résumé of useful formulas in queuing' theory, Operations Res. 5, 161 (1957). M 19, 106 Saaty, T. L., Mathematical methods of operations research (McGraw Hill Book Co., Inc., N.Y., 1958). M 21,225

- Samuels, J. C., Response of a simply supported Timoshenko beam to a purely random Gaussian process, J. Appl. Mech. 25, 496 (1958). M 20, 925

- Samuels, J. C., On stochastic linear systems, J. Math. Phys. 38, $83(1959 / 60)$. M 21,1125

- Savelli, M., (See P. Faure) C.R. Acad. Sci. Paris P44, 2371 (1957).

- Sawaragi, Y., The statistical studies on the response of automatic control systems with a nonlinear element of zero-memory type. I., Equivalent gains of non-linear element to an amplitude modulated signal in the presence of Gaussian noise. Tech. Rep. Engrg. Res. Inst. Kyoto Univ. 8, 95 (1958)

M 20, 126

Saxer, W., Versicherungsmathematik. 2ter Teil. Mit einem Anhang von H. Jecklin, Die Grundlehren der mathematischen Wissenschaften, Bd. 98. (Springer-Verlag, Berlin, 1958.)

M 20, 500

Scheffler, H., Strahlenoptische Ausbreitung in Medien mit statistisch verteilten Inhomogenitäten. I. Unregelmässige Refraktion als Markoff-Prozess, Astr. Nachr. 284, 227 (1958). M 20,1020

Scheffler, H., Strahlenoptische Ausbreitung in Medien mit statistisch verteilten Inhomogenitäten. II. Streuung in kleine Winkelbereiche, Astr. Nachr. 284, 269 (1959).
M $\mathbf{2 1}, 179$ 
- Scott,E. L. (SeeJ. Neyman), Science 130, 303(1959). Sedrakyan, L. G., Two problems of the statistical strength theory, Akad. Nauk Armyan. SSR Dokl. 26, 135 (1958).

M 21, 443

Šefl, O., On stability of a randomized linear system, Sci. Sinica \%, 1027 (1958).

M 21, 117 Shannon, C. E., Probability of error for optimal codes in a Gaussian channel, Bell. System Tech. J. 38, 611 (1959).

M $\mathbf{2 1}, 362$

Shenton, L. R., (See J. G. Skellam) J. Roy. Statist. Soc., Ser. B 19, 64 (1957).

Siforov, V. I., Parameters of binary coding systems (Translated by M. D. Friedman, 572 California St., Newtonville 60, Mass., 1956).

M 19, 374

Silverman, R. A., Scattering of plane waves by locally homogeneous dielectric noise, Proc. Cambridge Philos. Soc. 54, 530 (1958).

M 20, 492

Silverman, R. A., On radio scattering by dielectric turbulence, Div. Electromag. Res., Inst. Math. Sci., New York Univ., Res. Rep. No. EM-98, 30 pp. (1956).

M 20, 228

- Silverman, R. A., (See I. Kay) Nuovo Cimento (10) 9, 626 (1958).

- Skellam, J. G., Distributions associated with random walk and recurrent events, J. Roy. Statist. Soc., Ser. B 19, 64 (1957).

M 19, 990

Skitovič, V. P., On characterizing Brownian motion, Teor. Veroyatnost. i Primenen. 1, 361 (1956).

M 19, 73

Smith, W. L., On the cumulants of renewal processes, Biometrika 46, 1 (1959).

M 21, 579

Sobol, I. M., (See V.S. Vladimirov) Vyčisl. Mat. 3, 130 (1958).

Solodov, A. V., Statistical analysis of nonstationary processes in linear systems by using inverse simulating devices, Avtomat. i Telemeh. 19, 312 (1958).

M $\mathbf{2 0}, 943$

Špaček, A., Sur une caractérisation algébrique des espaces métriques, Bull. Acad. Polon. Sci. Sér. Sci. Math. Astr. Phys. 6, 445 (1958).

M $\mathbf{2 0}, 582$

Sprott, D. A., Probability distributions associated with distinct hits on targets, Bull. Math. Biophys. 19, 163 (1957).

M 19, 587

- Steinhaus. H., On the comparison of two production processes and the rule of dualism, Colloq. Math. 5, 103 (1957).

M 19, 1205

Stoller, D. S., Some queuing problems in machine maintenance, Naval Res. Logist. Quart. 5, 83 (1958)

M 20, 61

- Sunahara, Y., (See Y. Sawaragi) Tech. Rep. Engrg. Res. Inst. Kyoto Univ. 8, 95 (1958).

Svešnikov, A. A., Determination of the probability characteristics of three-dimensional seawaves, $I z v$. Akad. Nauk SSSR. Otd. Tehn. Nauk. Meh. Mašinostr., 1959, 32 (1959).

M 21, 584

Swensson, O., An approach to a class of queuing problems, Operations Res. 6, 276 (1958).

M 20, 60

von Sydow, L., Some aspects on the variations in traffic intensity, Teleteknik 1, 58 (1957).

M 19, 623
Takács, L., On the generalization of Erlang's formula, Acta Math. Acad. Sci. Hungar. 8, 419 (1956).

M 19, 623

Takács, L., A probability method for the treatment of the secondary electron emission, Magyar Tud. Akad. Mat. Fiz. Oszt. Közl. 6, 199 (1956).

M 20, 1107

Takács, L., On stochastic processes which occur in the theory of particle counters, Magyar Tud. Akad. Mat. Fiz. Oszt. Közl. 6, 369 (1956).

M 20, 227

Takács, L., On limiting distributions concerning a sojourn time problem, Acta Math. Acad. Sci. Hungar. 8, 279 (1957). M 20,464

Takács, L., On a probability problem concerning telephone traffic, Acta Math. Acad. Sci. Hungar. 8, 319 (1957).

M $\mathbf{2 0}, 465$

Takács, L., On a queueing problem concerning telephone traffic, Acta Math. Acad. Sci. Hungar. 8, 325 (1957).

M $\mathbf{2 0}, 465$

Takács, L., Über die wahrscheinlichkeitstheoretische Behandlung der Anodenstromschwankungen von Elektronenröhren, Acta Phys. Acad. Sci. Hungar. \%, 25 (1957).

M 19, 710

Takács, L., On a certain waiting time problem, Magyar Tud. Akad. Mat. Fiz. Oszt. Közl. \%, 183 (1957).

M $\mathbf{2 0}, 227$

Takács, L., Sojourn time problems, Magyar Tud. Akad. Mat. Fiz. Oszt. Közl. \%, 371 (1957).

M 20,465

Takács, L., On a generalization of the renewal theory, Magyar Tud. Akad. Mat. Kutató. Int. Közl. 2, 91 (1957).

M 21,580

Takács, L., Some probability questions in the theory of telephone traffic, Magyar Tud. Akad. Mat. Fiz. Oszt. Közl. 8, 151 (1958).

M 20, 465

Takács, L., On a coincidence problem concerning telephone traffic, Acta Math. Acad. Sci. Hungar. 9, 45 (1958).

M $\mathbf{2 0}, 465$

Takács, L., On a combined waiting time and loss problem concerning telephone traffic, Ann. Univ. Sci. Budapest. Eötvös. Sect. Math. 1, 73 (1958).

M 21, 317

Takács, L., On the limiting distribution of the number of coincidences concerning telephone traffic, Ann. Math. Statist. 30, 134 (1959). M 21, 317

Tanaka, H., On limiting distributions for one-dimensional diffusion processes, Bull. Math. Statist. $\mathbf{7}$, 84 (1957).

M 19, 470

Tanner, J. C., A simplified model for delays in overtaking on a two-lane road, J. Roy. Statist. Soc., Ser. B 20, 408 (1958).

M 20, 1019

'Tel'ksnis, L. A. (See Yu. P. Leonov), Avtomat. $i$ Telemeh. 18, 985 (1957).

- Thomson, W. T., The response of mechanical systems to random excitation, J. Appl. Mech. 24, 248 (1957).

M 19, 77

Tihonov, V. I., Effect of fluctuations in simplest parametric systems, Avtomat. $i$ Telemeh. 19, 717 (1958).

M $\mathbf{2 0}, 263$

- Tukey, J. W. (See R. B., Blackman), Bell System Tech. J. 3\%, 185 (1958). 
- Tukey, J. W. (See R. B., Blackman), Bell System Tech. J. 3\%, 485 (1958).

Uematu, T., On the traffic control at an intersection controlled by the repeated fixed-cycle traffic lights, Ann. Inst. Statist. Math., Tokyo 9, 87 (1958).

M 19, 894

Ueno, S., The probabilistic method for problems of radiative transfer. II. Milne's problem, Astrophys. J. 126, 413 (1957). M 20,736 Ulam, S. M., Infinite models in physics, Proc. Symposia in Appl. Math. VII, pp. 87-95. (McGrawHill Book Co., N.Y., 1957).

M 19, 779

Ullmo, J., Théorie des probabilitiés et mécanique, quantique, Publ. Inst. Statist. Univ. Paris 6, 251 (1957).

M 21, 350

Urbanik, K., Filtering of stationary generalized stochastic processes, Sci. Record (N.S.) R, 43 (1958).

M 21, 1124

- Van Meter, D. (See D. Middleton), J. Soc. Indust. Appl. Math. 4, 86 (1956).

- Vasudevan, R. (See A. Ramakrishnan), Astrophys. J. 126, 573 (1957).

- Vladimirov, V. S., Computation of the least eigenvalue of the Peierls equation by the Monte Carlo method, Vyčisl. Mat. 3, 130 (1958). M 21, 184

Vorob'ev, A. P., Some investigations on the rolling of a ship by the methods of the theory of probability, Leningrad. Gos. Univ. Uč. Zap. 21\%. Ser. Mat. Nauk 31, 3 (1957).

M 19, 779

Watanabe, S., A study of ergodicity and redundancy based on intersymbol correlation of finite range, Trans I.R.E. PGIT-4, 85 (1954). M 19, 470

- Wheeler, R. C., (See H. P. Galliher) Operations Res. 6, 264 (1958).

Wiener, N., Nonlinear problems in random theory (John Wiley \& Sons, Inc., N.Y., 1958).

M 20, 1201

Wilkinson, R. I., Theories for toll traffic engineering in the U.S.A., The Bell System Technical Journal 35, 421 (1956).

M 19, 516

- Wing, G. M., (See R. Bellman) Proc. Nat. Acad. Sci. U.S.A. 43, 517 (1957).

Winsten, C. B., Geometric distributions in the theory of queues, J. Roy. Statist. Soc., Ser. B 21 , 1 (1959).

M 21, 1238

Wishart, D. M. G., A queuing system with servicetime distribution of mixed chi-squared type,

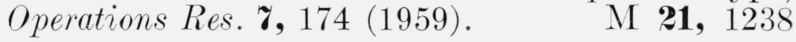

Yaglom, A. M. and I. M., Probability and information (Gosudarstv. Izdat. Tehn.-Teor. Lit., Moscow, 160 pp., 1957).

M 19, 990

Zitek, F., Courants d'entrée singuliers, Časopis Pèst. Mat. 83, 41 (1958).

M 20, 227

Zitek, F., Zur Theorie der ordinären nachwirkungsfreien Folgen, Czechoslovak Math. J. 8, 448 (1958).

M 21, 72

-Zubrzycki, S., (See H. Steinhaus) Colloq. Math. 5, 103 (1957).

\section{Frequency Functions, Moments, and Graduation}

Andreoli, G., Aspetto gruppale e funzionale delle medie, Giorn. Mat. Battaglini (5) 5 (85), 12 (1957).

M 19, 990

Andreoli, G., Medie e loro processi iterativi, Giorn. Mat. Battaglini (5) 5 (85), 52 (1957). M 19, 990

Aoyama, H., Sampling fluctuations of the test reliability, Ann. Inst. Statist. Math., Tokyo 8, 129 (1957).

M 19, 991

Bartholomew, D. J., Tests for randomness in a series of events when the alternative is a trend, J. Roy. Statist. Soc., Ser. B 18, 234 (1956). M 19, 1094

Barut, A. O., Distribution functions for noncommuting operators, Phys. Rev. (2) 108, 565 (1957).

M 19, 1131

Basu, D., A note on the multivariate extension of some theorems related to the univariate normal distribution, Sankhyā 17, 221 (1956). M 19, 471

Bazarov, I. P., Equations with variational derivatives and distribution functions for systems with complicated interaction, Dokl. Akad. Nauk SSSR (N.S.) 110, 38 (1956).
M 19, 186

Bennett, B. M., Certain multivariate distributions in the presence of intraclass correlation, J. Indian Soc. Agric. Statist. \%, 70 (1955).

M 19, 73

- Bhattacharyya, M. N., (See P. V. Krishna Iyer) J. Indian Soc. Agric. Statist. \%, 187 (1955).

Blumen, I., On the ranking problem, Psychometrika 22, 17 (1957).

M 19, 991

Bonferroni, C., I valori mediani e la teoria della misura, Giorn. Mat. Finanz. (4) 1, 89 (1955).

M 19, 372

Brown, R. L., Bivariate structural relation, Biometrika 44, 84 (1957).

M 19, 186

Brown, R. L., Multivariate linear structural relations, Biometrika 45, 136 (1958). M 19, 1094

- Brunk, H. D., Minimizing intergrals in certain classes of monotone functions, Pacific J. Math. $\%$ 833 (1957).

M 19, 289

Bulmer, M. G., Approximate confidence limits for components of variance, Biometrika 44, 159 (1957).

M 19, 73

Burkholder, D. L., On a class of stochastic approximation processes, Ann. Math. Statist. 2\%, 1044 (1956).

M 19, 71

Cornish, E. A., The sampling distributons of statistics derived from the multivariate $t$ - distribution, Austral. J. Phys. 8, 193 (1955). M 19, 1204

Corsten, L. C. A., Partition of experimental vectors connected with multinomial distributions, Biometrics 13, 451 (1957).

M 19, 1095

Cox, D. R., Some statistical methods connected with series of events, J. Roy. Statist. Soc., Ser. B 1\%, 129 (1955). M 19, 1094 Craig, A. T., (See R. V. Hogg') Sankhya 1\%, 209 (1956).

Čżan, L., On the precise distribution of A. N. Kolmogoroff and its asymptotic analysis, Acta Math. Sinica 6, 55 (1956). 
- Czechowski, T., et al, Statistical tables (Edited by Wieslaw Sadowski, Państwowe Wydawnictwo Naukowe, Warsaw, 1957).

M 19, 780

Darwin, J. H., The difference between consecutive members of a series of random variables arranged in order of size, Biometrika 44, 211 (1957).

M 19, 186

David, F. N., Reciprocal Bernoulli and Poisson variables, An. Fac. Ci. Porto 3\%, 200 (1953).

M 19, 187

Ewing, G. M. (See H. D. Brunk), Pacific J. Math. g, 833 (1957).

Farchi, V., Le curve degeneri del sistema algebrico delle parabole dei minimi quadrati, Rend. Mat. e Appl. (5) 15, 291 (1956).

M 19, 780

Faure, P., Déduction de certaines propriétés statistiques d'une fonction aléatoire stationnaire isotrope définie dans un espace à plusieurs dimensions de l'étude de sa trace sur une courbe de cet espace, C.R. Acad. Sci. Paris 244, 998 (1957). M 19, 693 - Fereday, F., (See R. L. Brown) Biometrika 45, 136 (1958).

Finney, D. J., The consequences of selection for a variate subject to errors of measurement, Rev. Inst. Internat. Statist. 24, 1 (1956). M 19, 781

Fisz, M., A limit theorem for empirical distribution functions, Bull. Acad. Polon. Sci. Cl. III. 5, 695 (1957).

M 19, 693

Fortunati, P., Rapporto di concentrazione, valori medi e schemi teorici di listribuzione massimante e minimante della variabilità, Scritti matematici in onore di Filippo Sibirani, pp. 109-122. (Cesare Zuffi, Bologna, 1957).

M 19, 188

Fox, C., Some applications of Mellin transforms to the theory of bivariate statistical distributions, Proc. Cambridge Philos. Soc. 53, 620 (1957).

M 19, 781

Frechet, M., Les tableaux de corrélation dont les marges et des bornes sont données, Ann. Univ. Lyon. Sect. A (3) \$0, 13 (1957). M 20,1020

Geary, R. C., Tests de la normalité, Ann. Inst. H. Poincaré 15, 35 (1956).

M 19, 473

Gold, L., Generalized Poisson distributions, Ann. Inst. Statist. Math., Tokyo 9, 43 (1957).

M 19, 1094

Good, I. J., On the serial test for random sequences, Ann. Math. Statist. 28, 262 (1957). M 19, 73

Gumbel, E. J., Distributions à plusieurs variables dont les marges sont données, C.R. Acad. Sci. Paris \$46, 2717 (1958).

M P0, 1020

Gurland, J., Distribution of the maximum of the arithmetic mean of correlated random variables, Ann. Math. Statist. 26, 294 (1955) M 19, 1204

Hájek, J., Inequalities for the generalised Student's distribution and their applications, Časopis Pěst. Mat. 82, 182 (1957).
Harley, B. I., Relation between the distributions of non-centra! $t$ and of a transformed correlation coefficient, Biometrika 44, 219 (1957). M 19, 472

Herzel, A., Sulle poligonali come distribuzioni generatrici di variabili divise in intervalli e sulla correzione dei primi due momenti, Statistica, Bologna 1\%, 414 (1957).

M 19, 1204

- Hogg, R. V., Sufficient statistics in elementary distribution theory, Sankhyā 17, 209 (1956).

M 19, 188

Hudimoto, H., A note on the probability of the correct classification when the distributions are not specified, Ann. Inst. Statist. Math., Tokyo 9, 31 (1957).

M 19, 1094

Huzurbazar, V. S., Sufficient statistics and orthogonal parameters, Sankhyā 17, 217 (1956). M 19, 471

Ihm, P., Berechnung von Integralen der $n$-dimensionalen Student-Verteilung mittels Steiltjesintegralpapier, Mitteilungsbl. Math. Statist. 9, 143 (1957).

M 19, 780

Ikenberry, E., Characteristics and convergence of Gram-Charlier series, Statistica, Bologna 1\%, 3 (1957).

M 19, 186

Jaeckel, K., Hauptachsentransformation der quadratischen Form für die Streuung, Z. Angew, Math. Mech. 37, 403 (1957).

M 19, 894

- Johnson, N. L., (See F. N. David) An. Fac. Ci. Porto 3\%, 200 (1953).

Johnson, N. L., A note on the mean deviation of the binomial distribution, Biometrika 44, 532 (1957).

M 19, 1204

- Kamat, A. R., (See Y. S. Sathe) Biometrika 44, 349 (1957).

- Kawashiro, T., (See Y. Watanabe) J. Gakugei Tokushima Univ. Nat. Sci. Math. 7, 53 (1956).

Keats, J. A., Estimation of error variances of test scores, Psychometrika \%2, 29 (1957). M 19, 329

- Khatri, C. G., (See K. V. Ramachandran) Ann. Math. Statist. 28, 802 (1957).

Kitagawa, T., Successive process of statistical interence associated with an additive family of sufficient statistics, Bull. Math. Statist. ' g, 92 (1957).

M 19, 693

Klega, V., On the truncated Maxwell's distribution, Apl. Mat. 2, 243 (1957).

M 19, 692

Konijn, H. S., A class of two-dimensional random variables and distribution functions, Sankhyā 18, 167 (1957).

M 19, 1087

Krishna Iyer, P. V., On some statistics comparing two binomial sequences, J. Indian Soc. Agric. Statist. $\mathbf{8 ,} 187$ (1955).

M 19, 329

Kullback, S., On the analysis of multiple regression in $k$ categories, Biometrika 44, 67 (1957).

M $\mathbf{1 9}, 186$

Laha, R. G., On some characterization problems connected with linear structural relations, Ann. Math. Statist. 28, 405 (1957). 
Lal, D. N., Distribution of the ratio of the logarithm of any one of the ranges of samples from a rectangular population to the sum of the logarithms of each of them, J. Indian Soc. Agric. Statist. 7, 179 (1955).

M 19, 73

Landenna, G., Osservazioni sulla connessione, Statistica, Bologna 1\%, 351 (1957).

M 19, 780

Leipnik, R. B., Moment generating functions of quadratic forms in serially correlated normal variables, Biometrika, 45, 198 (1958). M 19, 1095

- Lieberman, G. J., (See G. J. Resnikoff) (Stanford University Press, Stanford, California, 1957).

Lilliefors, H. W., A hand-computation determination of kill probability for weapons having spherical lethal volume, Operations Res. 5, 416 (1957).

M 19, 471

Linnik, Yu. V., Determining the probability distribution by a statistics distribution, Teor. Veroyatnost. i Primenen. 1, 466 (1956). M 19, 693

Linnik, Yu. V., A remark on Cramer's theorem on the decomposition of the normal law, Teor. Vercyatnost. i Primenen. 1, 479 (1956).

M 19, 894

Linnik, Yu. V., On "determining" statistics; a generalization of the problem of moments, Dokl. Akad. Nauk SSSR (N.S.) 113, 974 (1957).

M 19, 894

de Lucia, L., Variabilità a due dimensioni, Fac. Sci. Statist. Demogr. Attuar. Ist. Calcolo Probab. Publ. no. 2,13 pp. (1956).

M 20,339

Mallows, C. L., Non-null ranking models. I. Biometrika 44, 114 (1957).

M 19, 330

Matusita, K., Decision rule, based on the distance, for the classification problem, Ann. Inst. Statist. Math., Tokyo 8, 67 (1956).

M 19, 186

- Mazur, P., (See I. Oppenheim) Physica 23, 197 (1957).

- Mazur, P., I. Density expansions of distribution functions. II. Density expansions in the grand canonical ensemble, Physica 23, 216 (1957).

M 19, 191

Mazzoni, P., Sulla curva di Amoroso per la distribuzione dei redditi, Scritti matematici in onore di Filippo Sibirani, pp. 181-193. (Cesare Zuffi, Bologna, 1957.)

M 19, 588

Medgyessy, P., Anwendungsmöglichkeiten der Analyse der Wahrscheinlichkeitsdichtefunktionen bei der Auswertung von Messungsergebnissen, $Z$. Angew. Math. Mech. 37, 128 (1957). M 19, 187

- Mishra, D., (See D. N. Lal) J. Indian Soc. Agric. Statist. \%, 179 (1955).

Moore, P. G., Transformations to normality using fractional powers of the variable, $J$. Amer. Statist. Assoc. 52, 237 (1957). $\quad$ M 19, 330

Oderfeld, J., On the concentration of distribution, Zastos. Mat. 3, 182 (1957).

M 19, 894

- Oppenheim, I., Density expansions of distribution functions. I. Virial expansion for finite closed systems; canonical ensemble, Physica R3, 197 (1957).

M 19, 191

- Oppenheim, I., (See P. Mazur) Physica 23, 216 (1957).
- Ramachandran, K. V., On a decision procedure based on the Tukey statistic, Ann. Math. Statist. 28, 802 (1957).

M 19, 991

- Resnikoff, G. J., Tables of the non-central t-distribution: density function, cumulative distribution function and percentage points, Stanford studies in mathematics and statistics, I. (Stanford University Press, Stanford, California, 1957.) M 19, 187

Ríos, S., Sur la notion d'estimateur consistant, Trabajos Estadist. \%, 287 (1956). M 19, 471

- Rosenblatt, H. M., (See S. Kullback) Biometrika 44, 67 (1957).

Roy, K. P., A note on the asymptotic distribution of likelihood ratio, Calcutta Statist. Assoc. Bull. \%, 73 (1957).

M 19, 187

Sarmanov, O. V., Maximum correlation coefficient (non-symmetrical case), Dokl. Akad. Nauk SSSR 121, 52 (1958).

M 20, 918

- Sathe, Y. S., Approximations to the distributions of some measures of dispersion based on successive differences, Biometrika 44, 349 (1957). M 19, 692

Schäffer, K. A., Der Likelihood-Anpassungstest, Mitteilungsbl. Math. Statist. 9, 27 (1957).

M 19, 187

Seal, K. C., Approximate distribution of certain linear function of order statistics, Sankhyā 1\%, 345 (1957).

M 19, 1095

- Sibuya, M., Tables of the probability density function of range in normal samples, Ann. Inst. Statist. Math., Tokyo 8, 155 (1957). M 19, 583

Somerville, P. N., Optimum sampling in binomial populations, J. Amer. Statist. Assoc. 52, 494 (1957).

M 19, 991

Steck, G. P., Limit theorems for conditional distributions, Univ. California Publ. Statist. 2, 237 (1957).

M 19, 986

Teichroew, D., The mixture of normal distributions with different variances, Ann. Math. Statist. 28, 510 (1957).

M 19, 693

Toda, H., (See M. Sibuya) Ann. Inst. Statist. Math., Tokyo 8, 155 (1957).

Trybula, S., On a problem of prognosis, Bull. Acad. Polon. Sci. Cl. III. 5, 859 (1957).

M 19, 991

Tukey, J. W., On the comparative anatomy of transformations, Ann. Math. Statist. 28, 602 (1957).

M 19, 986

Tumanyan, S. H., Asymptotic distribution of $\chi^{2}$ criterion when the size of observations and the number of groups simultaneously increase, Teor. Veroyatnost. i Primenen. 1, 131 (1956). M 19, 467

Utz, W. R., (See H. D. Brunk) Pacific J. Math. \%, 833 (1957).

Wendel, J. G., Comment on "The distance to the origin of a certain point set in $E^{n}$ ", Proc. Amer. Math. Soc. 8, 413 (1957).

M 19, 58

- Watanabe, Y., On the compound normal distributions, J. Gakugei Tokushima Univ. Nat. Sci. Math. \%, 53 (1956).
M 19, 588 
- Wajiki, I., (See Y. Watanabe) J. Gakugei Tokushima Univ. Nat. Sci. Math. \%, 53 (1956).

White, J. S., Approximate moments for the serial correlation coefficient, Ann. Math. Statist. 28, 798 (1957).

M 19, 991

Wintner, A., Stratifications of Cauchy's "stable" transcendents and of Mittag-Leffler's entire functions, Amer. J. Math. 80, 111 (1958).

M 19, 1176

Wishart, J., An approximate formula for the cumulative z-distribution, Ann. Math. Statist. 28, 504 (1957).

M 19, 471

\section{Theory of Estimation and Testing of Hypoth-} eses, Sampling Distributions, and Theory of Sample Surveys

- Adhikari, B. P., Distance, discrimination et résumé exhaustif, Publ. Inst. Statist. Univ. Paris 5, 57 (1956).

M 19, 329

Adke, S. R., Generalized affinity and a class of distance functions, Proc. Nat. Inst. Sci. India. Part A 25, 104 (1959).

M 21, 1127

- Aggarwal, Om P., Truncation and tests of hypotheses, Ann. Math. Statist. 30, 230 (1959).

M 21, 319

- Aggarwal, Om P., Tables of the cumulative distribution functions of samples from symmetrically truncated normal distributions, Ann. Inst. Statist. Math. Tokyo 11, 55 (1959). M 21, 979 Agnew, R. P., Asymptotic expansions in global central limit theorems, Ann. Math. Statist. 30, 721 (1959)

M 21, 1239

- Aitchison, J., Maximum-likelihood estimation of parameters subject to restraints, Ann. Math. Statist. 29, 813 (1958).

M $\mathbf{2 0}, 229$

Aivvazyan, S. A., A comparison of the optimal properties of the Neyman-Pearson and the Wald sequential probability ratio tests, Teor. Veroyatnost. i Primenen. 4, 86 (1959). M 21,181

Akaike, H., Monte Carlo method applied to the solution of simultaneous linear equations, $A n n$. Inst. Statist. Math., Tokyo \%, 107 (1956).

M 19, 1096

Akaike, H., On optimum character of von Neumann's Monte Carlo model, Ann. Inst. Statist. Math., Tokyo \%, 183 (1959).

M 19, 1096

Allen, W. R., Inference from tests with continuously increasing stress, Operations Res. \%, 303 (1959).

M $\mathbf{2 1}, 982$

Anderson, T. W., An introduction to multivariate statistical analysis, (John Wiley \& Sons, Inc., New York, 1958).

M 19, 992

Anderson, T. W., Some scaling models and estimation procedures in the latent class model, The Harald Cramér volume, pp. 9-38. (John Wiley \& Sons, New York, 1959.)

M 21, 1408

Andreoli, G., Aspetto gruppale e funzionale delle medie, Giorn. Mat. Battaglini (5) 5 (85), 12 (1957).

M 19, 990

Andreoli, G., Medie e loro processi iterativi, Giorn. Mat. Battaglini (5) 5 (85), 52 (1957). M 19, 990
Angström, K. H., An asymptotic expansion of bias in a non-linear function of a set of unbiased characteristics from a finite sample, Skand. Aktuarietidskr. 41, 40 (1958).

M 21, 1126

Anscombe, F. J., Dependence of the fiducial argument of the sampling rule, Biometrika 44, 464 (1957).

M 19, 693

Armitage, P., Numerical studies in the sequential estimation of a binomial parameter, Biometrika $\mathbf{4 5}$, 1 (1958).

M 19, 1096

Askovitz, S. I., Graphic methods based upon properties of advancing centroids, J. Amer. Statist. Assoc. 54, 668 (1959).

M 21, 1126

Bahadur, R. R., On unbiased estimates of uniformly minimum variance, Sankhyā 18, 211 (1957).

M 19, 1096

Bahadur, R. R., Examples of inconsistency of maximum likelihood estimates, Sankhyā $\mathbf{2 0}, 207$ (1958).

M 21, 1128

Bailey, N. T. J., On estimating the latent and infectious periods of measles. I., Biometrika 43, 15 (1956).

M 19, 932

Bailey, N. T. J., On estimating the latent and infectious periods of measles. II., Biometrika 43, 322 (1956).

M 19, 932

Bailey, N. T. J., Significance tests for a variable chance of infection in chain-binomial theory, Biometrika 43, 332 (1956).

M 19, 932

Banerjee, D. P., Estimating the parameters of a censored or truncated distribution, Statistica 19, 211 (1959).

M 21,1241

Banerjee, S. K., A lower bound to the probability of Student's ratio, Sankhyā 18, 391 (1957).

M 19, 1204

Banerjee, S. K., Expressions for the lower bound to confidence coefficients, Sankhyā 21, 127 (1959).

M 21,1241

- Bargmann, R. E., (See S. N. Roy) Ann. Math. Statist. 29, 491 (1958).

Barra, J. R., Sir R. A. Fisher's "Statistical methods and scientific inference", Publ. Inst. Statist. Univ. Paris 6, 3 (1957).

M 19, 780

Bartholomew, D. J., A test of homogeneity for ordered alternatives, Biometrika 46, 36 (1959).

M 21, 581

Barton, D. E., A comparison of two sorts of test for a change of location applicable to truncated data, J. Roy. Statist. Soc., Ser. B 19, 119 (1957).

M 19, 1204

- Barton, D. E., Multiple runs, Biometrika 44, 168 (1957).

M 19, 70

Barton, D. E., The Modality of Neyman's contagious distribution of type A, Trabajos Estadist. 8, 13 (1957).

M 19, 188

Bašarin, G. P., The use of the chi-square criterion as a test for the independence of events, Dukl. Akad. Nauk SSSR (N.S.) 11\%, 167 (1957). M 20,64

Basmann, R. L., A generalized classical method of linear estimation of coefficients in a structural equation, Econometrica 25, 77 (1957). M 19, 74

Basu, D., A note on the theory of unbiased estimation, Ann. Math. Statist. 26, 345 (1955). M 19, 1204 
Basu, D., On statistics independent of sufficient statistics, Sankhyā 20, 223 (1958).

M 21, 832

Basu, D., On sampling with and without replacement, Sankhyā 20, 287 (1958).

M 21,837

Bennett, B. M., On confidence limits for the ratio of regression coefficients, Ann. Inst. Statist. Math., Tokyo 8, 41 (1956).

M 19, 74

Bennett, B. M., Tests for linearity of regression involving correlated observations, Ann. Inst. Statist. Math., Tokyo 8, 193 (1957).

M 19, 694

Bennett, B. M., Note on the method of inverse sampling, Trabajos Estadist. 8, 29 (1957).

M 19, 189

Beyer, O., Über zwei Anwendungen des Satzes von Ljapounoff der Wahrscheinlichkeitsrechnung, Wiss. Z. Hochsch. Schwermaschinenbau Magdeburg ?, 7 (1958).

M 21,1307

Birnbaum, Z. W., On an inequality due to S. Gatti., Metron 19, 243 (1958).

M 20, 1107

Bliss, C. I., Bioassay from a parabola, Biometrics 13, 35 (1957).

M 19, 330

- Bliss, C. I., Negative binomial distributions with a common $k$, Biometrika 45, 37 (1958).

M 19, 1096

Block, E., Numerical considerations for the stratification of variables following a logarithmic normal distribution, Skand. Aktuarietidskr. 41, 185 (1958).

M 21,1128

Block, H. D., Estimates of error for two modifications of the Robbins-Monro stochastic approximation process, Ann. Math. Statist. 28, 1003 (1957).

M 20, 64

Blom, G., Statistical estimates and transformed betavariables (John Wiley and Sons, Inc., New York, 1958).

М 20, 339

Blum, M., On exponential digital filters, J. Assoc. Comput. Mach. 6, 283 (1959). M 21,362

Blyth, C. R., Note on relative efficiency of tests, Ann. Math. Statist. 29, 898 (1958). M 20, 814

Blyth, C. R., Note on estimating information, Ann. Math. Statist. 30, 71 (1959).

M 21,362

Bonferroni, C., I valori mediani e la teoria della misura, Giorn. Mat. Finanz. (4) 1, 89 (1955).

M 19, 372

Borenius, G., On the distribution of the extreme values in a sample from a normal distribution, Skand. Aktuarietidskr. 41, 131 (1958).

M 21, 1127

Boyd, A. V., Bounds for Mills' ratio for the type III population, Ann. Math. Statist. 29, 926 (1958).

M $\mathbf{2 0}, 1107$

Boyd, A. V., The harmonic mean of independent $\gamma(1 / 2)$ variates, Simon Stevin 33, 34 (1959). M 21, 832

Breitenberger, E., Remarks on the least-squares reduction of angular correlation data, Proc. Phys. Soc., Sect. A 69, 489 (1956).

М 20719

Breny, H., Sur quelques problèmes d'analyse statistique posés par la physique des microcorpuscules. I. Distributions de Poisson et mesures relatives, Ann. Soc. Sci. Bruxelles. Sér. I. \%1, 135 (1957).

M 19, 895

Briggs, F. E. A., On problems of estimation in Leontief models, Econometrica 25, 444 (1957).

M 19, 781
Broadbent, S. R., Examination of a quantum hypothesis based on a single set of data, Biometrika 43, 32 (1956).

M 19, 588

Brownlee, K. A., A note on the effects of nonresponse on surveys, J. Amer. Statist. Assoc. 5\%, 29 (1957).

M 19, 475

Brudno, A. L., On a dispersion proof of the method of least squares, Mat. Sb. N.S. 43 (85), 37 (1957).

M 20, 63

Burkholder, D. L., On the existence of a best approximation of one distribution function by another of a given type, Ann. Math. Statist. 30, 738 (1959).

М 21, 979

Cansado, E., Sampling without replacement from finite populations, Trabajos Estadist. 8, 3 (1957).

М 19, 590

Carlson, P. G., Tests of hypothesis on the exponential lower limit, Skand. Aktuarietidskr. 41, 47 (1958).

М $\boldsymbol{2 1 ,} 980$

Carlson, P. G., A recurrence formula for the mean range for odd sample sizes, Skand. Aktuarietidskr. 41, 55 (1958).

M 21, 1126

Castellano, V., Sulla suddivisione in intervalli di una variabile già divisa in intervalli nella ipotesi che la distribuzione delle frequenze sia una poligonale, Scritti matematici in onore di Filippo Sibirani, pp. 63-80. (Cesare Zuffi, Bologna, 1957.)

М 21,180

Chakravarti, I. M., On a relation between canonical correlations and partial canonical correlations, Calcutta Statist. Assoc. Bull. 5, 185 (1954).

M 19, 895

Chanda, K. C., On some simplification in the construction of similar regions, Calcutta Statist. Assoc. Bull. 8, 159 (1959).

M 21, 727

Chatterjee, S. K., On an extension of Stein's twosample procedure to the multi-normal problem, Calcutta Statist. Assoc. Bull. 8, 121 (1959).

М 21, 834

Chiang, C. L., On regular best asymptotically normal estimates, Ann. Math. Statist. 2\%, 336 (1956).

M 19, 694

Clarke, A. B., Maximum likelihood estimates in a simple queue, Ann. Math. Statist. 28, 1036 (1957).

M 20,60

Clark, F. E., Truncation to meet requirements on means, J. Amer. Statist. Assoc. 52, 527 (1957).

M 19, 781

Cochran, W. G., Analysis of covariance: Its nature and uses, Biometrics 13, 261 (1957). M 19, 895

Cohen, A. C., On the solution of estimating equations for truncated and censored samples from normal populations, Biometrika 44, 225 (1957). M 19, 330

Cohen, A. C., Restriction and selection in multinormal distributions, Ann. Math. Statist. 28, 731 (1957).

M 19, 895

- Cohen, B. H., (See J. M. Sakoda) Psychometrika 22, 83 (1957).

Cohen, L., On mixed single sample experiments, Ann. Math. Statist. 29, 947 (1958). M 20, 1021

- Cornfield, J., Average values of mean squares in factorials, Ann. Math. Statist. 2\%, 907 (1956).

M 19, 331 
Cornish, E. A., An application of the Kronecker product of matrices in multiple regression, Biometrics 13, 19 (1957).

M 19, 895

Correa Pólit, H., Statistical inference about the parameters of nonnormal populations (confidence intervals), Trabajos Estadist. 9, 118 (1958).

M 20, 1108

Coulmy, G., Analyse et prédiction des marées, C.R. Acad. Sci. Paris 246, 1960 (1958).

M 20,64

Cox, D. R., The regression analysis of binary sequences, J. Roy. Statist. Soc., Ser. B 20, 215 (1958).

M 20, 918

Cox, D. R., Some problems connected with statistical inference, Ann. Math. Statist. 29, 357 (1958).

M $\mathbf{2 0}, 231$

Creasy, M. A., Analysis of variance as an alternative to factor analysis, J. Roy. Statist. Soc., Ser. B 19, 318 (1957).

M 19, 1095

Dall'Aglio, G., (See B. Michetti) Statistica, Bologna 1\%, 159 (1957)

van Dantzig, D., The development of mathematical statistics during the last ten years, Statistica, Neerlandica 9, 233 (1955).

M 19, 991

Darwin, J. H., On corrections to the chi-squared distribution, J. Roy. Statist. Soc., Ser. B 20, 387 (1958).

M 21, 580

Das, S. C., The numerical evaluation of a class of integrals. II., Proc. Cambridge Philos. Soc. 5\%, 442 (1956)

M 19, 983

- David, F. N., (See D. E. Barton) Biometrika 44, 168 (1957).

David, F. N., The z-test and symmetrically distributed random variables, Biometrika 46, 123 (1959).

M 21,443

David, H. A., Tournaments and paired comparisons, Biometrika 46, 139 (1959).

M 21, 228

Deal, R. B., (See F. A. Graybill) Biometrics 15, 543 (1959).

DeGroot, M. H., Unbiased sequential estimation for binomial populations, Ann. Math. Statist. 30, 80 (1959).

M 20, 1206

Deming, W. E., On the problem of matching lists by samples, J. Amer. Statist. Assoc. 54, 403 (1959).

M 21, 834

Dempster, A. P., Generalized $\mathrm{D}_{\mathrm{n}}^{+}$statisties, Ann. Math. Statist. 30, 593 (1959).

M 21,1126

DeNovellis, M., Some applications and developments of Gatti-Birnbaum inequality, Metron 19, 245 (1958).

M 20, 1107

Des Raj, On estimating parametric functions in stratified sampling designs, Sankhyā 1\%, 361 (1957).

M 19, 897

Des Raj, Some remarks on sampling with replacement, Ann. Math. Statist. 99, 550 (1958).

M 20, 228

- Dhruvarajan, P. S., A note on moments and cumulants, Math. Student 25, 27 (1957). $\quad$ M 20, 719

Diamond, S., Information and error: An introduction to statistical analysis (Basic Books, Inc., New York, 1959).

M 21, 727

- Diederich, G. W., A general least squares solution for successive intervals, Psychometrika 22, 159 (1957).

M 19, 589
Dixon, W. J., Estimates of the mean and standard deviation of a normal population, Ann. Math. Statist. 28, 806 (1957).

M 19, 992

- Doornbos, R., On slippage tests. I. A general type of slippage test and a slippage test for normal variates, Nederl. Akad. Wetensch. Proc. Ser. A 61Indag. Math. 20, 38 (1958).

M 20,720

- Doornbos, R., On slippage tests. II. Slippage tests for discrete variates, Nederl. Akad. Wetensch. Proc. Ser. A 61-Indag. Math. 20, 47 (1958).

M 20, 720

Dorogovcev, A. Ya. Confidence intervals in estimation of parameters, Dopovid Akad. Nauk Ukrain. RSR 1959, 355 (1959).

M 21,581

DuBois, P. H., Mulivariate correlational analysis (Harper \& Brothers, New York, 1957). M 19, 990

Dupač, V., On the Kiefer-Wolfowitz approximation method, Clasopis Pést. Mat. 82, 47 (1957).

M 19, 693

Durbin, J., Testing for serial correlation in systems of simultaneous regression equations, Biometrika 44, 370 (1957).

M 19, 991

Dwass, M., On several statistics related to empirical distribution functions, Ann. Math. Statist. 29, 188 (1958).

M $\mathbf{2 0}, 339$

Dwass, M., Multiple confidence procedures, Ann. Inst. Statist. Math. Tokyo 10, 277 (1959).

M $\mathbf{2 1}, 1128$

Dwyer, P. S., Generalizations of a Gaussian theorem, Ann. Math. Statist. 29, 106 (1958). $\quad$ M 20, 62

van Eeden, C., Maximum likelihood estimation of partially or completely ordered parameters, Math. Centrum Amsterdam. Statist. Afdeling. Rep. S 207, 17 pp. (1956).

M 19, 781

van Eeden, C., Maximum likelihood estimation of partially or completely ordered parameters. II., Nederl. Akad. Wetensch. Proc. Ser. A $60=$ Indag. Math. 19, 201 (1957).

M 19, 781

van Eeden, C., Note on two methods for estimating ordered parameters of probability distributions, Nederl. Akad. Wetensch. Proc. Ser. A $60=$ Indag. Math. 19, 506 (1957).

M 21, 318

van Eeden, C., A least squares in equality for maximum likelihood estimates of ordered parameters, Nederl. Akad. Wetensch. Proc. Ser. A $\mathbf{6 0}=$ Indag. Math. 19, 513 (1957).

M 21, 319

van Eeden, C., Testing and estimating ordered parameters of probability distributions (Mathematical Centre, Amsterdam, 1958).

M 21, 319

Ekman, G., An approximation useful in univariate stratification, Ann. Math. Statist. 30, 219 (1959).

M 21, 78

- Elfving, G., Item selection procedures for item variables with a known factor structure, Psychometrika 24, 189 (1959).

М 21, 1127

van Elteren, Ph., Some rank correlation methods, Statistica, Neerlandica 10, 177 (1956). M 19, 187

Fabian, F., On some fundamental questions of

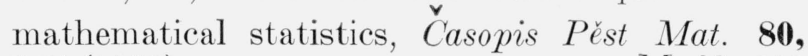
387 (1955). 
Fabian, V., Some modifications of interval estimation and choice of number of observations for a binomial random variable, Apl. Mat. 4, 35 (1959). M 21, 581

- Faxer, P., (See H. Wold) Ann. Math. Statist. 28, 265 (1957).

Federighi, E. T., Extended tables of the percentage points of Student's $t$-distribution, J. Amer. Statist. Assoc. 54, 683 (1959).

M 21, 1240

Fend, A. V., On the attainment of Cramér-Rao and Bhattacharyya bounds for the variance of an estimate, Ann. Math. Statist. 30, 381 (1959).

M 21, 833

Ferguson, T., A method of generating best asymptotically normal estimates with application to the estimation of bacterial densities, Ann. Math. Statist. 29, 1046 (1958).

M 20, 1108

Fernandes Costa, M. A., Note on the problem of comparison of the means of two normal universes, Gaz. Mat., Lisboa 1\%, 9 (1956).

M 20, 230

Finney, D. J., The efficiencies of alternative estimators for an asymptotic regression equation, Biometrika 45, 370 (1958).

M $\mathbf{m 1 , 5 8 1}$

Fisher, R. A., Recent progress in experimental design, Colloque tenu à Genève, 12-15 juillet 1939 (Institut International de Coopération Intellectuelle, Paris, 1945.) M $\mathbf{2 1}, 978$

Fisher, R. A., Mathematical probability in the natural sciences, Metrika $\mathbf{2 , 1}$ (1959). $\quad$ M $\mathbf{P 1}, 180$

Fisher, W. D., On grouping for maximum homogeneity, J. Amer. Statist. Assoc. 53, 789 (1958).

M 21,74

- Fix, E., The restricted chi-square test, The Harald Cramér volume, pp. 92-107. (John Wiley \& Sons, New York, 1959.)

M $\mathbf{2 1}, 1242$

- Foster, F. G., Upper percentage points of the generalized beta distribution. I., Biometrika 44, 237 (1957).

M 19, 188

Foster, F. G., Upper percentage points of the generalized beta distribution. II., Biometrika 44, 441 (1957).

M 19, 781

Foster, F. G., Upper percentage points of the generalized beta distribution. III., Biometrika $\mathbf{4 5}$, 492 (1958).

M 20, 1115

Fraser, D. A. S., Most powerful rank-type tests, Ann. Math. Statist. 28, 1040 (1957). M 20, 65

Frechet, M., Les tableaux de corrélation dont les marges et des bornes sont données, Ann. Univ. Lyon. Sect. A (3) 20, 13 (1957). $\quad$ M 20, 1020

Garner, N. R., Curtailed sampling for variables, J. Amer. Statist. Assoc. 53, 862 (1958). M 20, 1021

Gart, J. J., An extension of the Cramér-Rao inequality, Ann. Math. Statist. 30, 367 (1959).

M 21, 979

Gautschi, W., Some remarks on systematic sampling, Ann. Math. Statist. 28, 385 (1957). M 19, 475

Geary, R. C., Tests de la normalité, Ann. Inst. H. Poincaré 15, 35 (1956).

M 19, 473

Ghurye, S. G., Note on sufficient statistics and twostage procedures, Ann. Math. Statist. 29, 155 (1958).

M 20, 814
Gjeddebaek, N. F., Contribution to the study of grouped observations. II. Loss of information caused by grouping of normally distributed observations, Skand. Aktuarietidskr. 39, 154 (1956).

M 19, 992

Gjeddebaek, N. F., Contribution to the study of grouped observations. III. The distribution of estimates of the mean, Skand. Aktuarietidskr. 40, 20 (1957).

M 20, 63

Gjeddebaek, N. F., Contribution to the study of grouped observations. IV. Some comments on simple estimates, Biometrics 15, 433 (1959).

M 21, 1241

Glasgow, M. O., Note on the factorial moments of the distribution of locally maximal elements in a random sample, Ann. Math. Statist. 30, 586 (1959).

M 21, 318

- Glasser, G. J. (See W. E. Deming) J. Amer. Statist. Assoc. 54, 403 (1959).

- Gnanadesikan, R. (See S. N. Roy) Biometrika 44, 399 (1957).

Gnanadesikan, R., Equality of more than two variances and of more than two dispersion matrices against certain alternatives, Ann. Math. Statist. 30, 177 (1959).

M 21, 445

Gnedenko, B., Über die Nachprüfung statistischer Hypothesen mit Hilfe der Variationsreihe, Bericht über die Tagung Wahrscheinlichkeitsrechnung und mathematische Statistik in Berlin, Oktober, 1954, pp. 97-107. (Duetscher Verlag der Wissenschaf-ten, Berlin, 1956.)

M 19, 331

Gnedenko, B. V., On certain problems of the theory of probability, Ukrain. Mat. Z. 9, 377 (1957).

M 19, 776

Goen, R. L. (See R. F. Tate) Ann. Math. Statist. 29, 755 (1958).

Good, I. J., Significance tests in parallel and in series J. Amer. Statist. Assoc. 53, 799 (1958).

M 21, 445

Goodman, L. A., The precision of unbiased ratiotype estimators, J. Amer. Statist. Assoc. 53, 491 (1958).

M $\mathbf{2 0}, 229$

Goodman, L. A., Simplified runs tests and likelihood ratio tests for Markoff chains, Biometrika 45, 181 (1958).

M 19, 1090

Goodman, L. A., On some statistical test for $m$ th order Markov chains, Ann. Math. Statist. 30, 154 (1959).

M $\mathbf{2 1}, 78$

Govidarajulu, Z., A note on the correlation coefficient of the bivariate gamma type distribution, $J$. Madras Univ. Sect. B 26, 639 (1956). M 20,467

- Graf, U., Formeln und Tabellen der mathematischen, Statistik. Berichtigter Neudruck (Springer-Verlag, Berlin-Göttingen-Heidelberg, 1958).

M 20, 339

Graybill, F. A., Idempotent matrices and quadratic forms in the general linear hypothesis, Ann. Math. Statist. 28, 678 (1957).

M 19, 1095

Graybill, F. A., Determining sample size for a specified width confidence interval, Ann. Math. Statist. 29, 282 (1958).

M 20, 63

- Graybill, F. A., Combining unbiased estimators, Biometrics 15, 543 (1959).

M 21, 1240 
Grenander, U., On the theory of mortality measurement. I, Skand. Aktuarietidskr. 39, 70 (1956). M 19, 188

Grenander, U., On heterogeneity in non-life insurance. II, Skand. Aktuarietidskr. 40, 153 (1957).

M 20, 941

Gumbel, E. J., Distributions à plusieurs variables dont les marges sont données, $C$. R. Acad. Sci. Paris 246, 2717 (1958).

M 20, 1020

Gumbel, E. J., 'The $m$ th range, J. Math. Pures Appl. (9) 38, $253(1959)$.

M $\mathbf{2 1 , 1 4 0 8}$

- Gupta, S. S., On a statistic which arises in selection and ranking problems, Ann. Math. Statist. 28, 957 (1957).

M 20, 63

$\checkmark$ Gupta, S. S., On selecting a subset which contains all populations better than a standard, Ann. Math. Statist. 29, 235 (1958).

$\mathrm{M} \mathbf{2 0}, 63$

Gutman, M., On estimating an integral from the square of the density, Zastos. Mat. 3, 329 (1958).

M 19, 993

- Guttman, I., (See Om P. Aggarwal) Ann. Inst. Statist. Math. Tokyo 11, 55 (1959).

- Guttman, I., (See Om P. Aggarwal) Ann. Math. Statist. 30, 230 (1959).

- Hájek, J., (See F. Fabian) Casopis Pěst. Mat. 80, 387 (1955).

Hájek, J., On the theory of ratio estimates, Apl. Mat. 3, 384 (1958).

M 20, 1208

Hájek, J., On the distribution of some statistics in the presence of intraclass correlation, Casopis Pěst. Mat. 83, 327 (1958).

M 21, 75

Haldane, J. B. S., A class of efficient estimates of a parameter, Bull. Inst. Internat. Statist. 23, part II, 231 (1951).

M 19, 588

Haldane, J. B. S., Almost unbiassed estimates of functions of frequencies, Sankhyā 1\%, 201 (1956).

M 19, 330

Hall, Wm. J., The most-economical character of some Bechhofer and Sobel decision rules, Ann. Math. Statist. 30, 964 (1959). M 21, 1409

- Halton, J. H., A method for increasing the efficiency of Monte Carlo integration, J. Assoc. Comput. Mach. 4, 329 (1957).

M 20, 231

Hanania, M. I., A generalization of the BushMostellar model with some significance tests, Psychometrika 24, 53 (1959).

M 21, 727

- Handscomb, D. C., (See J. H. Halton) J. Assoc. Comput. Mach. 4, 329 (1957).

Harris, B., Determining bounds on integrals with applications to cataloging problems, Ann. Math. Statist. 30, 521 (1959).

M 21, 319

- Hartley, H. O., (See L. A. Goodman) J. Amer. Statist. Assoc. 53, 491 (1958).

Hellwig, Z., Determining linear regression parameters by means of the two point method, Zastos. Mat. 3, 66 (1956).

M 19, 472

Hendricks, W. A., The mathematical theory of sampling (The Scarecrow Press, New Brunswick, N.J., 1956).

M 19, 993

- Henning, H., (See U. Graf) (Springer-Verlag, Berlin-Gottingen-Heidelberg, 1958).
Herzel, A., Sulle poligonali come distribuzioni generatrici di variabili divise in intervalli e sulla correzione dei primi due momenti, Statistiac, Bologna 1\%, 414 (1957).

M 19, 1204

- Hess, I., (See L. Kish) J. Amer. Statist. Assoc. 54, 416 (1959).

Hodges, J. L., Jr., The significance probability of the Smirnov two-sample test, Ark. Mat. 3, 469 (1958).

M 20, 596

Hodges, J. L., Fitting the logistic by maximum likelihood, Biometrics 14, 453 (1958).

M 20, 1108

- Hodges, J. L., Jr., (See E. Fix) The Harald Cramér volume, pp. 92-107. (John Wiley \& Sons, New York, 1959).

- Hoeffding, W., Distinguishability of sets of distributions. (The case of independent and identically distributed chance variables), Ann. Math. Statist. 29, 700 (1958).

M 20, 340

Hoel, P. G., Efficiency problems in polynomial estimation, Ann. Math. Statist. 29, 1134 (1958).

M 20, 1206

Hudimoto, H., On the distribution-free classification of an individual into one of two groups, Ann. Inst. Statist. Math., Tokyo 8, 105 (1956). M 19, 472

- Ikeda, N., Notes on some relations between the distributions and sufficient statistics, Mem. Fac. Sci. Kyиsyu Univ. Ser. A 12, 12 (1958). M 20, 466 Ishii, G., On the exact probabilities of Renyi's tests, Ann. Inst. Statist. Math. Tokyo 11, 17 (1959).

M 21, 1240

Isii, K., On a method for generalizations of Tchebycheff's inequality, Ann. Inst. Statist. Math. Tokyo 10, 65 (1959).

M $\mathbf{2 1}, 832$

James, G. S., On moments and cumulants of systems of statistics, Sankhyā 20, 1 (1958). M 21,443

Jasper, S. J., A note on the standard deviation, Bul. Inst. Politehn. Iasi (N.S.) 3, 39 (1957). M 20, 918

- Jílek, M., Coefficients for the determination of one-sided tolerance limits of normal distribution, Ann. Inst. Statist. Math. Tokyo 11, 45 (1959).

M $\mathbf{2 1}, 1240$

- Johler, J. R., Mean absolute value and standard deviation of the phase of a constant vector plus a Rayleigh-distributed vector, J. Res. Nat. Bur. Standards 62, 183 (1959).

M 21, 444

Johnson, N. L., Optimal sampling for quota fulfilment, Biometrika 44, 518 (1957).

M 19, 1205

Johnson, N. L., The mean deviation, with special reference to samples from a Pearson type III population, Biometrika 45, 478 (1958).

M 20, 1108

Jones, H. L., How many of a group of random numbers will be usable in selecting a particular sample?, J. Amer. Statist. Assoc. 54, 102 (1959).

M 21, 318

Joshi, D. D., (See B. P. Adhikari) Publ. Inst. Statist. Univ. Paris 5, 57 (1956).

Kamat, A. R., A sampling experiment to verify the approximate theoretical distributions of mean successive difference, Calcutta Statist. Assoc. Bull. \%, 17 (1956).

M 19, 589 
Kamat, A. R., Moments of a certain distribution, Math. Student 25, 147 (1957).

M 20, 596

Kamat, A. R., Hypergeometric expansions for incomplete moments of the bivariate normal distribution, Sankhyā $\mathbf{2 0}, 317$ (1958).

M 21, 1127

Kamat, A. R., Incomplete moments of the trivariate normal distribution, Sankhyā 20, 321 (1958).

M 21, 1127

Kapur, M. N., A property of the optimum solution suggested by Paulson for the $K$-sample slippage problem for the normal distribution, J. Indian Soc. Agric. Statist. 9, 179 (1957).

M 21, 322

- Karlin, S., Distributions possessing a monotone likelihood ratio, J. Amer. Statist. Assoc. 51, 637 (1956).

M 21, 580

Kendall, M. G., A course in multivariate analysis, Griffin's Statistical Monographs \& Courses, no. 2, 185 pp. (Hafner Publishing Company, New York, 1957.)

M 19, 1093

- Kesten, H., Some remarks on the calculation of the expectation and the spread of the number of inconsistencies in a fixed rank correlation scheme, Statistica, Neerlandica 10, 197 (1956). M 19, 187

Kesten, H., Accelerated stochastic approximation, Ann. Math. Statist. 29, 41 (1958).

M $\mathbf{2 0}, 63$

Keyfitz, N., Estimates of sampling variance where two units are selected from each stratum, J. Amer. Statist. Assoc. 52, 503 (1957).

M 19, 783

- Khamis, S. H., (See Des Raj) Ann. Math. Statist. 29, 550 (1958).

Khatri, C. G., On conditions for the forms of the type $\mathrm{XAX}^{\prime}$ to be distributed independently or to obey Wishart distribution, Calcutta Statist. Assoc. Bull. 8, 162 (1959).

M 21, 832

- Kiefer, J., Some properties of generalized sequential probability ratio tests, Ann. Math. Statist. 28, 57 (1957).

M 19, 333

- Kiefer, J., On the deviations of the empiric distribution function of vector chance variables, Trans. Amer. Math. Soc. 8\%, 173 (1958).

M 20, 915

- Kiefer, J., Asymptotic minimax character of the sample distribution function for vector chance variables, Ann. Math. Statist. 30, 463 (1959).

M 21, 1240

- Kimball, A. W., Approximate linearization of the incomplete $\beta$-function, Biometrika 46, 214 (1959).

M 21, 180

$\checkmark$ Kish, L., On variances of ratios and their differences in multi-stage samples, J. Amer. Statist. Assoc. 54, 416 (1959).

M 21, 833

Kitagawa, T., The operational calculus and the estimations of functions of parameter admitting sufficient statistics, Bull. Math. Statist. 6, 95 (1956).

M 19, 330

Kitagawa, 'T., Successive processes of statistical controls. II., Mem. Fac. Sci. Kyushu Univ. Ser. A 13, 1 (1959).

M 21, 727

Kiveliovitch, M., L'estimation de la part du hasard, Publ. Sci. Tech. Ministere de l'Air, Notes Tech. No. $g$ g' (Paris, 1958).

M 21, 319

- Klamkin, M. S., (See D. J. Newman) Amer. Math. Monthly 66, 50 (1959).
- Klepikov, N. P., Non-linear confluence analysis, Teor. Veroyatnost. i Primenen. 2, 473 (1957).

M 20, 64

Klett, G. W., (See R. F. Tate, J. Amer. Statist. Assoc. 54, 674 (1959).

Kloos, $\boldsymbol{J}$., The determination of a factor of safety on the basis of a single probability parameter, Svenska Aeroplan A.B. Tech. Note No. 37 (1957).

M 19, 1096

Kordonskiǐ, H. B., On a distribution for the number of spoiled articles in shipments Teor. Veroyatnost. i Primenen. 3, 354 (1958).

M 21, 447

Koźniewska, I., Comparison of the efficiency of drawing samples with and without replacement when the variance of the general population is unknown Colloq. Math. 4, 232 (1957).

M 19, 475

Kramer, C. Y., Extension of multiple range tests to group correlated adjusted means, Biometrics $\mathbf{1 3}$, 13 (1957).

M 19, 331

Krishna Iyer, P. V., A theorem on factorial moments and its applications, Ann. Math. Statist. 29, 254 (1958).

M $\mathbf{2 0}, 62$

Kshirsagar, A. M., Bartlett decomposition and Wishart distribution, Ann. Math. Statist. 30, 239 $(1959)$.

M 21, 444

Kudô, A., On the distribution of the maximum value of an equally correlated sample from a normal population, Sankhyā 20, 309 (1958). M 21, 978

Kullback, S., Information theory and statistics (John Wiley and Sons, Inc., New York, 1959).

M 21, 444

Kulldorff, G., On the conditions for consistency and asymptotic efficiency of maximum likelihood estimates, Skand. Aktuarietidskr. 40, 129 (1957).

M 20, 719

Kulldorff, G., Maximum likelihood estimation of the mean of a normal random variable when the sample is grouped, Skand. Aktuarietidskr. 41, 1 (1958).

M 21,1241

Kulldorff, G., Maximum likelihood estimation of the standard deviation of a normal random variable when the sample is grouped, Skand. Aktuarietidskr. 41, 18 (1958).

M $\mathbf{2 1}, 1241$

Kulldorff, G., A problem of maximum likelihood estimation from a grouped sample, Metrika $\mathbf{2 ,} 94$ (1959).

M 21, 1241

Landenna, G., Osservazioni sulla connessione, Statistica, Bologna 17, 351 (1957). M 19, 780 - Leach, E., (See A. W. Kimball) Biometrika 46, 214 (1959).

- Lehmann, E. L., (See E. Fix) The Harald Cramér volume, pp. 92-107. (John Wiley \& Sons, New York, 1959.)

Lehmann, E. L., Testing statistical hypotheses (John Wiley \& Sons, Inc., New York 1959). M 21, 1242

Leslie, D. C. M., Determination of parameters in the Johnson system of probability distributions, Biometrika 46, 229 (1959).

M 21, 75

Levine, J., Monomial-monomial symmetric function tables, Biometrika 46, 205 (1959). $\quad$ M 21, 75

- Líkař, O., (See M. Jílek) Ann. Inst. Statist. Math. Tokyo 11, 45 (1959). 
Lindley, D. V., Binomial sampling schemes and the concept of information, Biometrika 44, 179 (1957).

M 19, 330

Lindley, D. V., Fiducial distributions and Bayes' theorem, J. Roy. Statist. Soc., Ser. B 20, 102 (1958).

M 20, 339

Linnik, Yu. V., A remark on Cramér's theorem on the decomposition of the normal law, Teor. Veroyatnost. i Primenen. 1, 479 (1956).

M 19, 894

Linnik, Yu. V., Some remarks on least squares in connection with direct and inverse location problems, Teor. Veroyatnost. i Primenen. 2, 349 (1957).

M 19, 1096

Linnik, Yu. V., On "determining" statistics; a generalization of the problem of moments, Dokl. Akad. Nauk SSSR (N.S.) 113, 974 (1957). M 19, 894

Liserre, G. O. G., A study of the $g$ and $d$ statistics, Rev. Un. Mat. Argentina 1\%, 91 (1955).

Lloyd, D. E., Note on a problem of estimation, Biometrika 46, 231 (1959).

M 21, 75

Lord, F. M., A significance test for the hypothesis that two variables measure the same trait except for errors of measurement, Psychometrika 22, 207 (1957).

M 19, 1095

de Lucia, L., Variabilità a due dimensioni, Fac. Sci. Statist. Demogr. Attuar. Ist. Calcolo Probab. Publ. no. 2, 13 pp. (1956).

M 20,339

Machek, J., On a two-sample procedure for testing Student's hypothesis using mean range, Apl. Mat. 4, $211(1959)$.

M 21, 834

MacKay, J. H., Asymptotically efficient tests based on the sums of observations, Ann. Math. Statist. 30, 806 (1959).

M 21, 1242

Madansky, A., 'The fitting of straight lines when both variables are subject to error, J. Amer. Statist. Assoc. 54, 173 (1959).

M 21, 319

Maniya, G. M., Quadratic estimate of the deviation of densities of a normal distribution in terms of sampling data, Soobšč. Akad. Nauk Gruzin. SSR 1\%, 201 (1956).

M 19, 694

Maniya, G. M., Quadratic error of estimation of density of a normal two-dimensional distribution in terms of sampling data, Soob̌̌c. Akad. Nauk Gruzin. SSR 20, 655 (1958).

M 20, 1020

- Marsaglia, G., (See F. A. Graybill) Ann. Math. Statist. 28, 678 (1957).

Mauldon, J. G., An inversion formula for a generalized transform, Mathematika 4, 146 (1957).

M 20, 194

Mauldon, J. G., A generalization of the beta-distribution, Ann. Math. Statist. 30, 509 (1959).

M 21, 318

Maxwell, A. E., Maximum likelihood estimates of item parameters using the logistic function, Pysychometrika 24, 221 (1959).

M 21, 1127

- McCarthy, P. J., (See F. F. Stephan) (John Wiley and Sons, Inc., New York, 1958).

- Messick, S. J., (See G. Diederich) Psychometrika 22, 159 (1957).

- Michetti, B., La differenza semplice media, Statistica, Bologna 1\%, 159 (1957).

M 19, 588
Mickey, M. R., Some finite population unbiased ratio and regression estimators, J. Amer. Statist. Assoc. 54, 594 (1959).

M 21, 1130

Mikami, M., On a multi-level sampling inspection plan for continuous production, Bull. Math. Statist. \%, 1 (1956).

M 21, 584

Mitra, S. K., Tables for tolerance limits for a normal population based on sample mean and range or mean range, J. Amer. Statist. Assoc. 52, 88 (1957).

M 19, 74

- Mitra, S. K., (See J. Roy) Sankhyā 18, 371 (1957).

Mitra, S. K., On the limiting power function of the frequency chi-square test, Ann. Math. Statist. 29, 1221 (1958).

M 21, 320

Moore, P. G., The transformation of a truncated Poisson distribution, Skand. Aktuarietidskr. 39, 19 (1956).

M 19, 472

Moore, P. G., The two-sample $t$-test based on range, Biometrika 44, 482 (1957). M 19,781

Moore, P. G., Interval analysis and the logarithmic transformation, J. Roy Statist. Soc., Ser. B 20, 187 (1958).

M 20, 596

Moran, P. A. P., The power of a cross-over test for the artificial stimulation of rain, Austral. J. Statist. 1, 47 (1959)

M 21, 1129

Moriguti, S., Notes on sampling inspection plans, Rep. Statist. Appl. Res. Un. Jap. Sci. Engrs. 3, 99 (1955).

M 19, 1205

- Morimoto, H., (See N. Ikeda) Mem. Fac. Sci. Kyusyu Univ. Ser. A 12, 12 (1958).

Moshman, J., A method for selecting the size of the initial sample in Stein's two sample procedure, Ann. Math. Statist. 29, 1271 (1958). M 20, 1206

Motoo, M., On the Hoeffding's combinatorial central limit theorem, Ann. Inst. Statist. Math., Tokyo 8, 145 (1957).

M 19, 694

Muniruzzaman, A. N. M., On measures of location and dispersion and test of hypotheses in a Pareto population, Calcutta Statist. Assoc. Bull. \%, 115 (1957).

M 19, 781

Murteira, B., On type A regions for Pólya distribubutions, Univ. Lisboa. Revista Fac. Ci. A (2) 6, 327 $(1957 / 58)$.

M 21, 445

Murthy, M. N., Ordered and unordered estimators in sampling without replacement, Sankhyō 18, 379 (1957).

M 20, 228

Narain, R. D., The general theory of sampling on successive occasions, bull. Inst. Internat. Statist. 24, 87 (1954).

M 19, 1205

Navrátil, J., Determination of the parameters of a compound distribution, Pokroky Mat. Fys. Astr. 3, 41 (1958).

M 20, 63

- Newman, D. J., Expectations for sums of powers, Amer. Math. Monthly 66, 50 (1959). M 21, 75

Neyman, J., Basic ideas and some recent results of the theory of testing statistical hypotheses, Colloque tenu à Genève, Juillet 1939, pp. 81-127. (Institut International de Coopération Intellectuelle, Paris, 1945.)

M 21, 978

Neyman, J., Current problems of mathematical statistics, Proc. Int. Cong. Math., Amsterdam, 1, 349. (North-Holland Publ. Co., Amsterdam, 1957.)
M 20, 228 
Nicholson, G. E., Estimation of parameters from incomplete multivariate samples, J. Amer. Statist. Assoc. 52, 523 (1957). $\quad$ M 19, 783

Nicholson, W. L., On the distribution of 2x2 random normal determinants, Ann. Math. Statist. 29, 575 (1958).

M 20, 62

Noether, G. E., Two confidence intervals for the ratio of two probabilities and some measures of effectiveness, J. Amer. Statist. Assoc. 52, 36 (1957).

M 19, 74

Nordbotten, S., On errors and optimal allocation in a census, Skand. Aktuarietidskr. 40, 1 (1957).

M 20, 69

Ochoa, J., Bounds for variance, Euclides, Madrid 16, 273 (1956).

M 20, 813

Okamoto, M., Some inequalities relating to the partial sum of binomial probabilities, Ann. Inst. Statist. Math. 10, 29 (1958).

M 20, 1020

Oktaba, W., On the linear hypothesis in the theory of normal regression, Eull. Acad. Polon. Sci. Sér. Sci. Math. Astr. Phys. 6, 75 (1958). M 20, 596

$\checkmark$ Olds, E. G., A comparison of tests on the mean of a logarithmico-normal distribution with known variance, WADC Tech. Note 55-249. (Wright Air Devel. Center, Wright-Patterson Air Force Bose, Ohio, 1955.)

M 19, 188

- Olkin, I., Unbiased estimation of certain correlation coefficients, Ann. Math. Statist. 29, 201 (1958).

M 20, 64

Olkin, I., Multivariate ratio estimation for finite populations, Biometrika 45, 154 (1958).

M 19, 1097

$\checkmark$ Ostle, B., Correlation between sample means and sample ranges, J. Amer. Statist. Assoc. 54, 465 (1959).

M 21, 832

- Owen, A. R. G. (See C. I. Bliss), Biometrika 45, 37 (1958).

- Owen, D. B. (See G. P. Steck), J. Amer. Statist. Assoc. 54, 689 (1959).

Pearson, E. S., Note on Mr. Quenouille's Edgeworth Type A transformation, Biometrika 46, 203 (1959).

M 21, 180

Petrov, A. A., Verification of statistical hypotheses on the type of a distribution based on small samples, Teor. Veroyatnost. i Primenen. 1, 248 (1956).

M 19, 76

- van Peype, W. F. (See van Elteren, Ph.), Statistica, Neerlandica 10, 177 (1956).

Pfanzagl, J., Ein kombiniertes Test \& KlassifkationsProblem, Metrika 2, 11 (1959).

Pillai, K C S On Hotelling's M 21, 181 of $\mathrm{T}^{2}$, Biometrika 46, 160 (1959).

Plackett, R. L Iinear estimation fre data, Ann. Math. Statist. 29, 131 (1958).

Plackett, R. L., The analysis of life test data Technometrics 1, 9 (1959). M 21,982

Pompilj, G., Lo schema delle prove ripetute a due stadi stratificati, Fac. Sci. Statist. Demogr. Attuar. Ist. Statist. Ist. Calcolo Probab. Publ. no 3, 22 pp. (1956).

M 19, 1097

- Pratt, J. W. (See I. Olkin), Ann. Math. Statist. 29, 201 (1958).
Pratt, J. W., Admissible one-sided tests for the mean of a rectangular distribution, Ann. Math. Statist. 29, 1268 (1958).

M 20, 1109

- Prins, H. J. (See R. Doornbos), Nederl. Akad. Wetensch. Proc. Ser. A $\mathbf{6 1}=$ Indag. Math. 20, 38 (1958).

- Prins, H. J. (See R. Doornbos), Nederl. Akad. Wetensch. Proc. Ser. A $\mathbf{6 1}=$ Indag. Math. 20, 47 (1958).

Quandt, R. E., Probabilistic errors in the Leontief system, Naval Res. Logist. Quart. 5, 155 (1958).

M $\mathbf{2 0}, 466$

Quandt, R. E., The estimation of the parameters of a linear regression system obeying two separate regimes, J. Amer. Statist. Assoc. 53, 873 (1958).

M $\mathbf{2 0}, 1108$

Quenouille, M. H., Tables of random observations from standard distributions, Eiometrika 46, 178 (1959).

M 21, 180

Quensel, C. E., Some sampling problems when a stratification variable follows a logarithmic normal distribution, Skand. Aktuarietidskr. 41, 177 (1958).

M 21,1128

Quensel, C., A contribution to the theory of correlation and regression in non-random samples, Lunds Univ. Arsskrift. N.F. Avd. 2. 54, 31 pp. (1958).

M 20, 67

Radhakrishna Rao, C., Sur une caractérisation de la distribution normale établie d' après une propriété optimum des estimations linéaires, Colloq. Intern. du Centre Nat. de la Recherche Sci., 8\%, 196 pp. (Paris, 1959).

M $\mathbf{2 1}, 833$

Radner, R., Minimax estimation for linear regressions, Ann Math. Statist. 99, 1244 (1958).

M 20, 1108

Raja Rao, B., On the relative efficiencies of BAN estimates based on doubly truncated and censored samples, Proc. Nat. Inst. Sci. India Part A $\mathbf{2 4}$, 366 (1958).

M 21, 833

Rajski, C., The selectivity of the parametric tests, Trans. 1st Prague Conf. on Inform. Theory, Stat'l. Decision Fns., Random Processes held at Liblice, Nov. 28-30, 1956, pp. 33-34. (Publ. House of the Czechoslovak Acad. Sci., Prague, 1957.)

M $\mathbf{2 0}, 814$

Ramachandran, K. V., On the studentized smallest chi-square, J. Amer. Statist. Assoc. 53, 868 (1958).

M 21, 75

Ramasubban, T. A., The generalized mean differences of the binomial and Poisson distributions, Biometrika 46, 223 (1959).

M 21, 444

Rangarajan, R., A note on two stage sampling, Sankhyā 1\%, 373 (1957).

M 19, 897

Rao, B. R., On an analogue of Cramér-Rao's inequality, Skand. Aktuarietidskr. 41, 57 (1958).

M 21, 979

Rao, C. R., Maxim likelihood estimation for the multinomial distribution, Sankhyā 18, 139 (1957).

M 21, 727

Raэ, C. R., Maximum likelihood estimation for the multinomial distribution with infinite number of cells, Sankhya $\mathbf{2 0}, 211$ (1958).

M 21, 1128 
Ray, W. D., A proof that the sequential probability ratio test (S.P.R.T.) of the general linear hypothesis terminates with probability unity, Ann. Math. Statist. 28, 521 (1957).

M 19, 472

Ray, W. D., Sequential confidence intervals for the mean of a normal population with unknown variance, J. Roy. Statist. Soc., Ser. B 19, 133 (1957).

M 19, 781

- Rees, D. H., (See F. G. Foster) Biometrika 44, 237 (1957).

Režný, Z., On tests of statistical hypotheses by gauging with respect to two limits, Apl. Mat. 4, 290 (1959).

M $\mathbf{2 1}, 1242$

Rider, P. R., The midrange of a sample as an estimator of the population midrange, J. Amer. Statist. Assoc. 52, 537 (1957).

M 19, 783

Rider, P. R., Generalized Cauchy distributions, Ann. Inst. Statist. Math. Tokyo 9, 215 (1958).

M 20, 228

Rider, P. R., Quasi-ranges of samples from an exponential population, Ann. Math. Statist. 30, 252 (1959).

M 21, 444

Robson, D. S., Applications of multivariate polykays to the theory of unbiased ratio-type estimation, J. Amer. Statist. Assoc. 52, 511 (1957). M 19, 1097

Rosett, R. N., A statistical model of friction in economics, Econometrica $\mathbf{2 \% ,} 263$ (1959). M 21, 886

Roy, J., On some tests of significance in samples from bipolar normal distributions, Sankhyā 14, 203 (1954).

M 19, 896

- Roy, J., Unbiased minimum variance estimation in a class of discrete distribution, Sankhya $\mathbf{1 8}, 371$ (1957).

M 19, 1096

- Roy, S. N., Further contributions to multivariate confidence bounds, Biometrika 44, 399 (1957).

M 19, 895

Roy, S. N., Some aspects of multivariate analysis (John Wiley and Sons Inc., New York, 1957).

M 19, 1093

- Roy, S. N., Tests of multiple independence and the associated confidence bounds, Ann. Math. Statist. 29, 491 (1958).

M 20, 65

- Rubin, H., (See S. Karlin) J. Amer. Statist. Assoc. 51, 637 (1956).

Rubin, H., Estimation of a regression line with both variables subject to error under an unusual identification condition, Ann. Math. Statist. 29, 606 (1958).

M $\mathbf{2 0}, 229$

- Runnenburg, J. Th., (See H. Kesten) Statistica, Neerlandica 10, 197 (1956).

Rybarz, J., Ein einfacher Beweis für das dem $\mathrm{X}^{2}$ Verfahren zugrundeliegende Theorem, Metrika 2 89 (1959).

M 21, 1127

Saitô, K., Maximum-likelihood estimate of proportion using supplementary information, Bull. Math. Statist. \%, 11 (1956).

M 19, 472

Saitô, K., Some results in the theory of sampling on successive occasions with partial replacement of units, Rep. Statist. Appl. Res. Un. Jap. Sci. Engrs. 4, 125 (1957)

M 19, 897

- Sakoda, J. M., Exact probabilities for contingency tables using binomial coefficients, Psychometrika 22, 83 (1957).

M 19, 73
- Samson, P., Jr., (See K. C. S. Pillai) Biometrika 46, 160 (1959).

Sankaran, M., On the non-central chi-square distribution, Biometrika 46, 235 (1959). M 21, 75

Sarmanov, O. V., Maximum correlation coefficient (non-symmetrical case), Dokl. Akad. Nauk SSSR 121, 52 (1958).

M 20,918

Savage, I. R., On the independence of tests of randomness and other hypotheses, J. Amer. Statist. Assoc. 52, 53 (1957).

M 19, 331

Saw, J. G., Estimation of the normal population parameters given a singly censored sample, Biometrika 46, 150 (1959).

M 21, 444

Schmetterer, L., Bemerkungen zur Theorie der erwartungstreuen Schätzfunktionen, Mitteilungsbl. Math. Statist. 9, 147 (1957).

M 20, 230

Schmid, P., On the Kolmogorov and Smirnov limit theorems for discontinuous distribution functions, Ann. Math. Statist. 29, 1011 (1958). M 21, 75

Schutzenberger, M. P., A propos de l'inégalité de Fréchet-Cramér, Publ. Inst. Statist. Univ. Paris y, 3 (1958).

M 21, 833

Sen. A. R., A simple design in sampling with varying probabilities, J. Indian Soc. Agric. Statistic. 7,57 (1955).

M 19, 475

Severo, N. C., (See E. G. Olds) WADC Tech. Note 55-249. (Wright Air Devel. Center, WrightPatterson Air Force Base, Ohio, 1955.)

Shenton, L. R., Moment estimators and maximum likelihood, Biometrika 45, 411 (1958). M $\mathbf{2 0 ,} 1107$

Shimada, S., Moments of order statistic drawn from exponential distribution, Rep. Statist. Appl. Res. Un. Jap. Sci. Engrs. 4, 153 (1957). M 21, 580

Siddiqui, M. M., Distribution of a serial correlation coefficient near the ends of the range, Ann. Math. Statist. 29, 852 (1958).

M 20, 62

Silverstone, H., Estimating the logistic curve, $J$. Amer. Statist. Assoc. 52, 567 (1957). M $\mathbf{2 0 ,} 339$

Silvey, S. D., (See J. Aitchison) Ann. Math. Statist. 29, 813 (1958).

Silvey, S. D., The Lindisfarne scribes' problem, $J$. Roy. Statist. Soc., Ser. B 20, 93 (1958). M 20, 340

Silvey, S. D., The Lagrangian multiplier test, Ann. Math. Statist. 30, 389 (1959).

M 21, 580

Simaika, J. B., On two characteristics of a random direction, Proc. Math. Phys. Soc. Egypt 5, no. 4, 113 (1956).

M 19, 1201

- Singal, M. K., (See P. S. Dhruvarajan) Math. Student 25, 27 (1957).

Siotani, M., The significance of the discordant variance estimates, Ann. Inst. Statist. Math., Tokyo \%, 39 (1955).

M 19, 1204

Siotani, M., The extreme value of the generalized distances of the individual points in the multivariate normal sample, Ann. Inst. Statist. Math. Tokyo. 10, 183 (1959).

M 21, 979

Sitgreaves, R., (See G. Elfving) Psychometrika 24, 189 (1959).

Smith, C. D., On the mathematics of simple correlation, Math. Mag. 32, 57 (1958). $\quad$ M 20,918

Smith, H. F., A multivariate analysis of covariance, Biometrics 14, 107 (1958).

M 19, 1095

Sobel, M., (See S. S. Gupta) Ann. Math. Statist. 28, 957 (1957). 
- Sobel, M., (See S. S. Gupta) Ann. Math. Statist. 29, 235 (1958).

- Sokolov, S. N., (See N. P. Klepikov) Teor. Veroyatnost. i Primenen 2, 473 (1957.)

- Solomon, H., (See G. Elfving) Psychometrika 24, 189 (1959).

Somermeijer, W. H., Substitute variables in correlation analysis, Statistica Neerlandica 11, 153 (1957).

M 19, 780

Sprott, D. A., The method of maximum likelihood applied to the Poisson binomial distribution, Biometrics, 14, 97 (1958).

M 20, 814

Srivastava, A. B. L., Effect of non-normality on the power function of $t$-test, Biometrika 45, 421 (1958).

M $\mathbf{2 0}, 1109$

Steck, G. P., A uniqueness property not enjoyed by the normal distribution, Ann. Math. Statist. 99, 604 (1958).

M 20,813

- Steck, G. P., Percentage points for the distribution of outgoing quality, J. Amer. Statist. Assoc. 54, $689(1959)$.

M 21, 1245

- Steck, G. P., (See B. Ostle) J. Amer. Statist. Assoc. 54, 465 (1959).

Steinhaus, H., The problem of estimation, Ann. Math. Statist. 28, 633 (1957).

M 19, 1095

- Stephan, F. F., Sampling opinions: an aralysis of survey procedure (John Wiley and Sons, Inc., New York, 1958).

M 20, 125

Stevens, W. L., Shorter intervals for the parameter of the binomial and Poisson distributions, Biometrika 44, 436 (1957).

M 19, 780

Stevens, W. L., Sampling without replacement with probability proportional to size, J. Roy. Statist. Soc., Ser. B 20, 393 (1958).

M 20, 1208

- St.-Pierre, J., The null distribution of the difference between the two largest sample values, Ann. Math. Statist. 2\%, 849 (1956).

M 21, 580

st.-Pierre, J., Distribution of linear contrasts of order statistics, Ann. Math. Statist. 29, 1264 (1958).

M \%0, 1020

Stuart, A., The asymptotic relative efficiencies of tests and the derivatives of their power functions, Skand. Aktuarietidskr. 3\%, 163 (1954). M 19, 1204

Stuart, A., Equally correlated variates and the multinormal integral, J. Roy. Statist. Soc., Ser. B 20, 373 (1958).

M $\mathbf{2 0}, 813$

Sukhatme, B. V., Testing the hypothesis that two populations differ only in location, Ann. Math. Statist. 29, 60 (1958). M 20, 340

Suzuki, Y., Discrete decision problems, Ann. Inst. Statist. Math., Tokyo 9, 131 (1958). M 20,64

Talacko, J., A note about a family of Perks' distribution, Sankhyā $\mathbf{2 0}, 323$ (1958).

M 21, 1239

- Tate, R. F., Minimum variance unbiased estimation for the truncated Poisson distribution, Ann. Math. Statist. 99, 755 (1958).

M $\mathbf{2 0}, 63$

- Tate, R. F., Optimal confidence intervals for the variance of a normal distribution, J. Amer. Statist. Assoc. 54, 674 (1959).

M $\mathbf{2 1}, 1241$

Tate, R. F., Unbiased estimation: Functions of location and scale parameters, Ann. Math. Statist. 30, 341 (1959).

M 21, 581
-Theil, H., On the efficiency of Wald's method of fitting straight lines, Rev. Inst. Internat. Statist. $\mathbf{2 4}$ 17 (1956)

M 19, 781

Thionet, P., Sur la variance de l'estimation d'une variance, C.R. Acad. Sci. Paris $\mathbf{2 4 5 ,} 2168$ (1957).

M 19, 1095

Thionet, P., Une généralisation de la variance d'échantillonnage dans le cas de tirages exhaustifs d'une urne, C.R. Acad. Sci. Paris $\mathbf{2 4 5 ,} 2464$ (1957).

M 19, 1095

Thionet, P., Représentation topologique des sondages, C.R. Acad. Sci. Paris \$26, 46 (1958).

M 19, 1095

Thionet, P., Sur les rapports entre divers concepts d'information, C.R. Acad. Sci. Paris $\mathbf{9 4 6 ,} 223$ (1958).

M $\mathbf{2 0}, 466$

Thionet, P., Sur les pertes d'information qui sont des fonctions de risque, C.R. Acad. Sci. Paris $\mathbf{2 1 6}, 367$ (1958).

M $\mathbf{2 0}, 229$

Thionet, P., Sur les pertes d'information imputables à certaines estimations biaisées, C.R. Acad. Sci. Paris \$46, 536 (1958).

M 20,229

Thionet, P., Sur une théori générale des pertes d'information par sondage, C.R. Acad. Sci. Paris $\mathbf{9 4 6}$, 692 (1958).

M $\mathbf{2 0}, 229$

Tiago de Oliveira, J., Estimators and tests for continuous populations with location and dispersion parameters, Univ. Lisboa. Revista Fac. Ci. A (2) 6, $121(1957 / 58)$.

M 21, 75

Tikkiwal, B. D., An application of the theory of multiphase sampling on successive occasions to surveys of live-stock marketing, J. Karnatak Univ. 1, $120(1956)$.

M 21, 728

Trybula, S., Some problems of simultaneous minimax estimation, Ann. Math. Statist. 29, 245 (1958).

M 20, 64

Trybula, S., On the minimax estimation of the parameters in a multinomial distribution, Zastos. Mat. 3, 307 (1958).

M $\$ 1,75$

- Tucker, L. R., (See G. W. Diederich) Psychometrika 22, 159 (1957).

Tucker, L. R., An inter-battery method of factor analysis, Psychometrika $\mathbf{2 3 , 1 1 1}$ (1958). M $\mathbf{2 0 , 1 0 2 1}$

- Tukey, J. W., (See J. Cornfield) Ann. Math. Statist. 2\%, 907 (1956).

Tukey, J. W., Sums of random partitions of ranks, Ann. Math. Statist. 98, 987 (1957). M 20,62

Tukey, J. W., Antithesis or regression?, Proc. Cambridge Philos. Soc. 53, 923 (1957).

M 19, 991

Tukey, J. W., A problem of Berkson, and minimum variance orderly estimators, Ann Math. Statist. 29, 588 (1958).

M $\mathbf{2 0}, 229$

Tulse, R., Sampling for variables with a very skew distribution, Appl. Statist. 6, 40 (1957). M 19, 76

Tumanyan, S. H., On the power of the chi-square criterion applied to the problem of two samples relative to "near" alternatives, Izv. Akad. Nauk Armyan. SSR. Ser. Fiz.-Mat. Nauk 11, 31 (1958). M 21,76

- Turner, F., (See J. Kloos) Svenska Aeroplan A.B. Tech. Note No. 3\%, 13 pp. (1957).

- Uchiyama, M., (See K. Yoneda) Yokohama Math. J. 4,99 (1956). 
Uhlmann, W., Eine wahrscheinlichkeitstheoretische Begründung der Integrationsformeln von NewtonCotes, Z. Angew. Math. Phys. 10, 189 (1959).

M 21,839

Ura, S., Minimax approach to a single sampling: inspection by attributes, Rep. Statist. Appl. Res. Un. Jap. Sci. Engrs. 3, 140 (1955). M 19, 1205

Ura, S., On Scheffé's analysis of variance for paired comparisons, Rep. Statist. Appl. Res. Un. Jap. Sci. Engrs. 4, 132 (1957).

M 19, 895

Vincze, I., Einige zweidimensionale Verteilungsund Grenzverteilungssätze in der Theorie der geordneten Stichproben, Magyar Tud. Akad. Mat. Kutató Int. Közl. 2, 183 (1957).

M 21, 725

Voelz, K., Über die Berechnung der Regressionskoeffizienten mit Hilfe von Orthogonalfunktionen, Mitteilungsbl. Math. Statist. 9, 113 (1957).

M 19, 1096

Wallace, D. L., Intersection region confidence procedures with an application to the location of the maximum in quadratic regression, Ann. Math. Statist. 29, 455 (1958).

M $\mathbf{2 0}, 339$

Walsh, J. E., Estimating population mean, variance, and percentage points from truncated data, Skand. Aktuarietidskr. 39, 47 (1956).

M 19, 330

- Walters, L. C., (See J. R. Johler) J. Res. Nat. Bur. Standards 6\%, 183 (1959).

Watson, G. S., The $\chi^{2}$ goodness-of-fit test for normal distributions, Biometrika 44, 336 (1957).

M 19, 895

Watson, G. S., On chi-square goodness-of-fit tests for continuous distributions, J. Roy. Statist. Soc., Ser. B 20, 44 (1958).

M 20, 1107

Weber, E., Das Ergebnis-Folge-Verfahren (Sequenzanalyse). Grundlagen und Anwendungen, Wiss. Z. Humboldt-Univ. Berlin. Math-Nat. Reihe $;$, $559(1957 / 58)$.

M 21,1242

Wegmüller, W., Das Grenzverhalten statistischer Prüfverteilungen, Mitt. Verein. Schweiz. Versich.Math. 58, 127 (1958).

M 20, 918

Weiler, H., Note on harmonic and geometric means, Austral. J. Statist. 1, 44 (1959).

M 21, 1408

Weiss, L., A note on confidence sets for random variables, Ann. Math. Statist. 26, 142 (1955).

M 19, 896

- Weiss, L., (See J. Kiefer) Ann. Math. Statist. 28, 57 (1957).

Weiss, L., The convergence of certain functions of sample spacings, Ann. Math. Statist. 28, 778 (1957).

M P0, 466

Weiss, L., The asymptotic power of certain tests of fit based on sample spacings, Ann. Math. Statist. 88, 783 (1957).

M 20, 466

Weiss, L., Limiting distributions of homogeneous functions of sample spacings, Ann. Math. Statist. 29, 310 (1958).

M 20, 813

Weiss, L., A test of fit for multivariate distributions, Ann. Math. Statist. 29, 595 (1958).

M 20, 64

Weiss, L., The limiting joint distribution of the largest and smallest sample spacings, Ann. Math. Statist. 30, 590 (1959).

M 21,319

Welch, B. L., "Student" and small sample theory, J. Amer. Statist. Assoc. 53, 777 (1958). M 20, 1021
Wesler, O., A classification problem involving multinomials, Ann. Math. Statist. 30, 128 (1959).

M 21,834

Whittle, P., On the smoothing of probability density functions, J. Roy. Statist. Soc., Ser. B 20, 334 (1958).

M $\mathbf{2 0}, 918$

Wijsman, R. A., Incomplete sufficient statistics and similar tests, Ann. Math. Statist. 99, 1028 (1958).

M 21, 980

Wijsman, R. A., On the theory of BAN estimates, Ann. Math. Statist. 30, 185 (1959). M $\mathbf{2 1 ,} 833$

Wilkinson, J. W., An analysis of paired comparison designs with incomplete repetitions, Biometrika 44, 97 (1957).

M 19, 473

Wilks, S. S., De l'application de la théorie des probabilitiés à certains problèmes d 'échantillonnage en statistique ma thématique, Colloque tenu à Genère, Juillet 1939, pp. 33-80. (Collection Scientifique. Institut International de Coopération Intellectuelle, Paris, 1945.)

M 21, 978

Wise, M. E., Formulae relating to single-sample inspection by attributes, Philips Res. Rep. 10, $97(1955)$

M 19, 1205

- Wold,H., On the specification error in regression analysis, Ann. Math. Statist. 28, 265 (1957). M 19, 74

Wolfowitz, J., The minimum distance method, Ann. Math. Statist. 28, 75 (1957). M 19, 472

- Wolfowitz, J., (See W. Hoeffding) Ann. Math. Statist. 29, 700 (1958)

- Wolfowitz, J., (See J. Kiefer) Trans. Amer. Math. Soc. 8\%, 173 (1958).

- Wolfowitz, J., (See J. Kiefer) Ann. Math. Statist. 30, 463 (1959)

- Yoneda, K., Some estimations in the case of relatively large class intervals, Yokohama Math. J. 4, 99 (1956).

M $\mathbf{2 0}, 719$

- Yzeren, J., (See H. Theil) Rev. Inst. Internat. Statist. 24, 17 (1956).

Zelen, M., The analysis of incomplete block designs, J. Amer. Statist. Assoc. 52, 204 (1957). M 19, 74

Ziaud-Din, M., The expression of $k$-statistic $k_{11}$ in terms of power sums and sample moments, Ann. Math. Statist. 30, 825 (1959). M 21, 1240

Zimmermann, K. F., Tabellen, Formeln und Fachausdrücke zur Variationsstatistik: Für Landwirtschaftswissenschaftler, Naturwissenschaftler, Mediziner und Ingenieure (VEB Deutscher Verlag der Wissenschaften, Berlin, 1959). M 21,318

Zindler, H., Über Faustregeln zur optimalen Schichtung bei Normalverteilung, Allg. Statist. Arch. 40, 168 (1956).

M 19, 189

Zinger, A. A., On independence of polynomial and quasipolynomial statistics, Dokl. Akad. Nauk SSSR (N.S.) 110, 319 (1956). M 19,991

- Zinger, A., (See J. St-Pierre) Ann, Math, Statist. 97, 849 (1956).

Zinger, A. A., Independence of quasi-polynomial statistics and analytical properties of distributions, Teor. Veroyatnost. i Primenen. 3, 265 (1958).

M 21, 181

Zoroa, P., Convolution of histograms, Trabajos Estadist. 9, 159 (1958).

M $\mathbf{2 1}, 318$

(Paper 67B2-97) 


\section{Publications of The National Bureau of Standards*}

\section{Selected Abstracts}

Perturbation method in a problem of waveguide theory, D. Fox and W. Magnus, J. Research NBS 67D (Radio Prop.) No. 2, 189 (Mar.-Apr. 1963). 70 cents.

The reflection coefficient for the basic mode in a widening. straight, two-dimensional waveguide is computed for small wave numbers by using the perturbation method with the electrostatic case as the unperturbed case. The problem is treated as a perturbed infinite system of inhomogeneous linear equations, and it is shown that the matrix of the unperturbed system (which corresponds to the electrostatic case) can be inverted explicitly by using conformal mappings and physically unrealistic modes. Questions of convergence are discussed, and other examples for application of the method are indicated.

Some wave functions and potential functions pertaining to spherically stratified media, C. T. Tai, J. Research NBS 67D (Radio Prop.) No. 2, 199 (Mar.-A pr. 1963). 70 cents.

The wave functions pertaining to a bilinearly stratified medium are presented in this paper. Solutions to the equation $\nabla \cdot[\kappa(r) \nabla \Psi]=0$ have been investigated for several profiles of $\kappa(r)$. An analysis is given to the equation $\nabla \cdot[\kappa \cdot \nabla \Psi]=-\rho / \epsilon_{0}$ which arises from the formulation of the quasi-static electric field in a homogeneous anisotropic medium.

Relative convergence of the solution of a doubly infinite set of equations, R. Mittra, J. Research $N B S$ 67D (Radio Prop.) No. 2, 245 (Mar.-Apr. 1963). 70 cents.

The paper deals with the relative convergence of a doublyinfinite set of equations pertaining to a boundary value problem in a waveguide. It is shown that the solution for the equations converges differently for different choices of the combination of the number of equations from the two sets. This is demonstrated by studying the convergence properties of the truncated set of equations as the number of equations is made indefinitely large. It is proven that the solution for the reflection coefficient becomes identical with the exact solution only with a particular choice of the ratio of the equations. This choice of the unique ratio is also shown to be consistent with the edge condition.

Realistic diatomic potential function, $H$. W. Woolley, $J$. chem. Phys. 37, No. 6, 1307-1316 (Sept. 1962).

An examination of recently published data for diatomic molecules as obtained by the Rydberg-Klein-Rees method has suggested a useful form for the potential-energy function. This form provides an inverse-power dependence at large distance and an extrapolation to infinity at the extreme of close approach. Relations are given to permit an arbitrarily close fit of observed spectroscopic constants. Theoretical formulas covering seven new $Y\left[{ }_{l i}\right]$ 's of the Dunham-Sandeman series for the rotating vibrator are included. Potential curves for $\mathrm{H}_{2}$ and $\mathrm{HF}$ are shown as examples, including effects of previously determined higher-order spectroscopic constants. Other applications of the new potential are also discussed.

Tests for contingency tables and Markov chains, S. Kullback, M. Kupperman, and H. H. Ku, Technometrics 4, No. 4, 573608 (Nov. 1962).

A number of useful tests for contingency tables and finite stationary Markov chains are presented in this paper based on the use of the notions of information theorv. A consistent and simple approach is used in developing the various test procedures and the results are given in the form of analysisof-information tables. Beginning with tests of hypotheses for a one-way table, tests of hypotheses of specified probabilities, independence, conditional independence, homogeneity of classifications, and symmetry are developed for contingency tables of two, three, four, and higher order classifications.
For the Markov chains, the tests include the hypotheses of a specified matrix of transition probabilities, Markovity, and homogeneity of several realizations of Markov chains. Worked examples are given throughout the paper. A table of $2 n \ln n$ for $n=1$ (1) 10,000 is appended for use in computation.

A molecular-orbital study of the geometry of $\mathbf{H O}_{2}$, M. E. Boyd, J. Chem. Phys. 37, No. 6, 1317-1325 (Sept. 1962).

Determination of the energv of $\mathrm{HO}_{2}$ as a function of the $\mathrm{HO}_{1} \mathrm{O}_{2}$ angle for fixed values of $R\left(\mathrm{H}-\mathrm{O}_{1}\right)$ and $R\left(\mathrm{H}-\mathrm{O}_{2}\right)$ has been carried out using LCAO-MO-SCF calculations on closedshell ions. All one-electron and one- and two-center twoelectron integrals used were evaluated accurately while three-center integrals were approximated. An isoscelestriangle configuration is favored by these calculations and gives a value of the ionization potential of $9.5 \mathrm{eV}$ and dissociation energy of $4.4 \mathrm{eV}$, consonant with experimental results. Approximation by means of ionic calculations used here is shown to be valid for the purposes of this discussion.

\section{Other NBS Publications}

\section{J. Research NBS 67A (Phys. and Chem.), No. 2 (Mar.-} Apr. 1963) 70 cents.

Third spectrum of palladium (Pd III). A. G. Shenstone.

Broadening of the rotational lines of carbon monoxide by $\mathrm{HCl}$ and argon. R. J. Thibault, J. H. Jaffe, and E. K. Plyler.

Theory of frustrated total reflection involving metallic surfaces. T. R. Young and B. D. Rothrock.

Quantitative metallography with a digital computer: application to a Nb-Sn superconducting wire. G. A. Moore and L. L. Wyman.

Moiré fringes produced by a point projection X-ray microscope. S. B. Newman.

Cyclic polyhydroxy ketones. I. Oxidation products of hexahydroxybenzene (benzenehexol). A. J. Fatiadi and H. S. Isbell.

Effect of pressure and temperature on the refractive indices of benzene, carbon tetrachloride, and water. R. M. Waxler and C. E. Weir.

Pressure-density-temperature relations of fluid para hydrogen from 15 to $100^{\circ} \mathrm{K}$ at pressures to 350 atmospheres. R. D. Goodwin, D. E. Diller, H. M. Roder, and L. A. Weber.

A method for determining the elastic constants of a cubic crystal from velocity measurements in a single arbitrary direction; application to $\mathrm{SrTiO}_{3}$. J. B. Wachtman, Jr., M. L. Wheat, and S. Marzullo.

\section{J. Research NBS 67A (Phys. and Chem.), No. 3 (May- June 1963) 70 cents.}

An absolute calibration of the National Bureau of Standards Thermal Neutron Flux. E. J. Axton.

Application of two interferometers in series to the measurement of length. I. C. Gardner.

Absorption bands of carbon dioxide from 5.3 to 4.6 microns. A. G. Maki, E. K. Plyler, and R. J. Thibault.

Infrared spectrum of the $\nu_{2}-\nu_{6}$ band of $\mathrm{C}^{13} \mathrm{C}^{12} \mathrm{H}_{6}$. W. J Lafferty and E. K. Plyler.

Self-broadening of carbon monoxide in the $2 \nu$ and $3 \nu$ bands E. K. Plyler and R. J. Thibault.

Thermodynamic properties of polyethylene predicted from paraffin data. M. G. Broadhurst.

Spectrophotometric determination of the thermodynamic $p \mathrm{~K}$ value of picric acid in water at $25{ }^{\circ} \mathrm{C}$. M. M. Davis and M. Paabo.

Purity analysis of highly purified materials by time-temperature cryometry. G. S. Ross and H. D. Dixon. 
Synthesis of isomers of eugenol. Morris, and W. B. Howe.

Analysis of families of curves. MeCrackin.

A controlled atmosphere chamber. C. L. Gordon and R. B. Johannsen.

J. Research NBS 67C (Eng. and Instr.), No. 2 (Apr.-June 1963) 75 cents.

Temperature dependence of the elastic constants of thoria specimens of varying porosity. S. Spinner, L. Stone, and F. P. Knudsen.

Residual stresses and their relaxation on the surfaces of sections cut from plastically deformed steel specimens. J. Newton.

Permeation rates of electrolytic hydrogen and deuterium through iron. J. W. Pitts.

Steady state heat conduction in cylinders with multiple continuous line heat sources. B. A. Peavy.

A radial-flow apparatus for determining the thermal conductivity of loose-fill insulations to high temperatures. D. R. Flynn.

Analysis of a microwave radiometer for precise standardization of noise sources. G. D. Ward and J. M. Richardson.

Realistic evaluation of the precision and accuracy of instrument calibration systems. C. Eisenhart.

\section{J. Research NBS 67D (Radio Prop.), No. 2 (Mar.-Apr. 1963 70 cents.}

The protection of frequencies for radio astronomy. R. L. Smith-Rose.

Radar reflections from the moon at $425 \mathrm{Mc} / \mathrm{s}$. G. H. Millman and Fred L. Rose.

Sunset and sunrise in the ionosphere: effects on the propagation of longwaves. J. Rieker.

Correction of atmospheric refraction errors in radio height finding. W. B. Sweezy and B. R. Bean.

Empirical determination of total atmospheric refraction at centimeter wavelengths by radiometric means. A. C Anway.

Propagation of radiofrequency electromagnetic fields in geological conductors. Volker Fritsch, translated from German by A. P. Barsis.

WWV reception in the arctic during ionospheric disturbances G. E. Hill and J. R. Herman.

Height-gain for VLF radio waves. J. R. Wait and K. P. Spies.

Perturbation method in a problem of waveguide theory. D. Fox and W. Magnus. (See above abstracts).

Some wave functions and potential functions pertaining to spherically stratified media. C. T. Tai. (See above abstracts.)

Radiation from a plasma-clad axially-slotted cylinder. W. V. T. Rusch.

Two- and three-loop superdirective receiving antennas. E. W. Seeley.

Hallén's method in the problem of a cavity-backed rectangular slot antenna. J. Galejs.

Relative convergence of the solution of a doubly infinite set of equations. R. Mittra. (See above abstracts.)

\section{J. Research NBS 67D (Radio Prop.), No. 3 (May-June 1963) 70 cents.}

Effects of radio wave propagation through mid-latitude 6300 A ares. J. R. Roach.

Comparison of observed atmospheric radio refraction effects with values predicted through the use of surface weather observations. B. R. Bean and G. D. Thayer

Ionospheric scattering effects in long-distance propagation. H. A. Whale.

Concerning solutions of the VLF mode problem for an anisotropic curved ionosphere. J. R. Wait.

On the statistical theory of electromagnetic waves in a fluctuating medium (1). K. Furutsu.

Reception of skywave signals near a coastline. J. B. Andersen.
Analysis and synthesis of nonuniform transmission lines or stratified layers. G. Latmiral, G. Franeschetti, and R. Vinciquerra.

Resonant characteristics of a corrugated sphere. J. R. Wait and C. M. Jackson.

Impedances of long antennas in air and in dissipative media. D. W. Gooch, C. W. Harrison, Jr., R. W. P. King, and T. T. Wu.

Reflection of VLF radio waves from an inhomogeneous ionosphere. Part I. Exponentially varying isotropic model. J. R. Wait and L. C. Walters.

NBS viscometer calibrating liquids and capillary tube viscometers, R. C. Hardy, NBS Mono. 55 (Dec. 26, 1962), 20 cents.

Periodicals received in the Library of the National Bureau of Standards, July 1962, N. J. Hopper, NBS Mono. 57 (Nov. 23, 1962), 25 cents (Supersedes NBS Circular 563 and the 1st supplement to NBS Circular 563).

Corrosion of steel pilings in soils, M. Romanoff, NBS Mono. 58 (Oct. 24, 1962), 20 cents.

Radiation quantities and units, International Commission on Radiological Units and Measurements (ICRU) Report 10a, 1962, NBS Handb. 84 (Nov. 14, 1962), 20 cents.

Handbook for CRPL ionospheric predictions based on numerical methods of mapping, S. M. Ostrow, NBS Handb. 90 (Dec. 21, 1962), 40 cents (Supersedes Cir. 465).

Report of the 47 th National Conference on Weights and Measures 1962, NBS Misc. Publ. 244 (Nov. 23, 1962), 75 cents.

Hydraulic research in the United States 1962, H. K. Middleton, NBS Misc. Publ. 245 (Oct. 26, 1962), \$1.00.

1962 Research Highlights of the National Bureau of Standards, Annual Report, NBS Misc. Publ. 246 (Dec. 1962), 70 cents.

Equipment characteristics and their relation to system performance for tropospheric communication circuits, A. F. Barghausen, F. O. Guiraud, R. E. McGavin, S. Murahata, and R. W. Wilber, NBS Tech. Note 103 (Jan. 15, 1963), $\$ 1.00$.

A tabulation of the thermodynamic properties of normal hydrogen from low temperature to $300^{\circ} \mathrm{K}$ and from 1 to 100 atmospheres, J. W. Dean, NBS Tech. Note 120 (Nov. 1961), 45 cents.

The thermodynamic properties of nitrogen from 114 to $540^{\circ}$ $\mathrm{R}$ between 1.0 and 3000 psia, Supplement A (British units), T. R. Strobridge, NBS Tech. Note 129A (Feb. 1963), 50 cents.

Coordinated color identifications for industry, K. L. Kelly, NBS Tech. Note 152 (Nov. 1962), 15 cents.

The thermodynamic properties of helium from 6 to $540^{\circ} \mathrm{R}$ between 10 and 1500 psia, D. B. Mann, NBS Tech. Note 154A (Jan. 1962), 50 cents.

Emission stabilization of thermionic diode noise sources, M. W. Randall and M. G. Arthur, NBS Tech. Note 160 (Sep. 1962), 15 cents.

Thermal balance in the $\mathrm{F}$ region of the atmosphere, D. C Hunt, NBS Tech. Note 162 (Sept. 1962), 50 cents.

The construction of calorimeters for the measurement of absorbed dose, B. Petree and G. Ward, NBS Tech. Note 163 (Nov. 1962), 25 cents.

Fading correlation bandwidth and short-term frequency stability measurements on a high-frequency transauroral path, J. L. Auterman, NBS Tech. Note 165 (Oct. 1962), 40 cents.

The following National Bureau of Standards Technical Note is available by purchase from the Office of Technical Services, U.S. Department of Commerce, Washington 25, D.C. (please order by PB number).

An atlas of whistlers and VLF emissions. A survey of VLF spectra from Boulder, Colorado, D. L. Jones, R. M. Gallet, J. M. Watts, and D. N. Frazer, NBS Tech. Note 166 (PB181454) (Jan. 1963), \$2.25.

The Hall effect, D. O. Webster, Student Quart. \& EE Dig. p. 82-86 (Sept. 1962).

Dimensional changes in complete dentures on drying, wetting and heating in water, G. C. Paffenbarger and W. T. Sweeney, J. Am. Dental Assoc. 65, No. 4, 495-505 (Oct. 1962). 
A variable-parameter direct-current switching filter, G. F. Montgomery, Proc. IRE 50, No. 9, 1986 (Sept. 1962).

The vapor phase radiolysis of propane- $d_{8}$ in the presence of other hydrocarbons, S. G. Lias and P. Ausloos, J. Chem. Phys. 37, No. 4, 877-883 (Aug. 15, 1962).

Synthesis of an immittance function with two negative impedance converters, S. B. Geller, IRE Trans. Circuit Theory CT-9, No. 3, 291 (Sept. 1962).

A note on the propagation of electromagnetic pulses over the earth's surface, J. R. Wait, Can. J. Phys. 40, 1264-1269 (1962).

Surface behavior of silver single crystals in fused sodium chloride, J. Kruger and K. H. Stern, J. Electrochem. Soc. 109, No. 10, 889-894 (Oct. 1962).

The spontaneous martensitic transformations in $18 \% \mathrm{Cr}$, 8\% Ni steels, R. P. Reed, Acta Met. 10, 865-877 (Sept. 1962).

A high speed pyrometer, G. A. Hornbeck, Book, Temperature, Its Measurement and Control in Science and Industry 3, Pt. 2, 425-428 (Reinhold Publ. Corp., New York, N.Y., 1962 ).

The viscous heating correction for fiscometer flows, E. A. Kearsley, Trans. Soc. Rheology VI, 253-261 (1962)

Wavelengths, energy levels, and pressure shifts in mercury 198, V. Kaufman, J. Opt. Soc. Am. 52, No. 8, 866-870 (Aug. 1962).

A network transfer theorem, G. F. Montgomery, IRE Trans. Audio AU-10, No. 3 (Mav-June 1962).

Strengthening of hot work die steels, C. R. Irish and S. J. Rosenberg, Trans. Quart. 55, No. 3, 613-623 (Sept. 1962).

Acoustical interferometer emploved as an instrument for measuring low absolute temperatures, G. Cataland and H. H. Plumb J. Acoust. Soc. Am. 34, No. 8, 1145-1146 (Aug. 1962)

Correlation of factors influencing the pressures generated in multi-anvil devices, J. C. Houck and U. O. Hutton, Am. Soc. Mech. Engrs. Paper 62-WA-254 (1962).

The temperature dependence of flow and fracture characteristics of an age-hardenable alloy, W. D. Jenkins and W. A. Willard, Trans. ASM 55, No. 3, 580-598 (Sept. 1962).

Rotational perturbations in CN I. Zero-field theory, optical Zeeman effect, and microwave transition probabilities, H. E. Radford and H. P. Broida, Phys. Rev. 128, No. 1, 231-242 (Oct. 1962).

The effect of experimental variable including the martensitic transformation on the low-temperature mechanical properties of austenitic stainless steels, C. J. Guntner and R. P. Reed, Trans. ASM 55, No. 3, 399-419 (Sept. 1962).

A new ionospheric multipath reduction factor (MRF), R. K. Salaman, IRE Trans. Commun. Systems CS-10, 220-222 (June 1962).

The effect of electrolytes on the sodium chromotropism of bix-(meso-2,3-diaminobutane)-nickel (II) ions, D. L. Leussing, J. Harris, and P. Wood, J. Phys. Chem. 66, 1544-1546 (Aug. 1962).

Characteristics of resistance strain gages, R. L. Bloss, Book, Semiconductor and Conventional Strain Gages, Ed. Mills Dean, III and R. D. Douglas, Chapt. VII, 123-142 (Academic Press, Inc.. New York, N.Y., Oct. 1962).

The photodisintegration of bismuth and the lead isotopes, E. G. Fuller and E. Hayward, Nuclear Phys. 33, 431-448 (1962).

Comparison of the relative acidic strengths of the isomeric dinitrophenols in benzene and water, M. M. Davis, J. Am. Ceram. Soc. 84, 3623-3627 (1962).

The basis of the measurement system, A. V. Astin, Proc. IRE 50, No. 614-616 (May 1962).

Predicting the performance of long distance tropospheric communication circuits, A. P. Barsis, K. A. Norton, and P. L. Rice, IRE Trans. Commun. Systems CS-10, 2-22 (Mar. 1962).

A neuron model which performs analog functions, D. R. Boyle, Proc. San Diego Symp. Biomedical Engr., San Diego, Calif., pp. 256-263 (June 19-21, 1962).

The system of $\mathrm{Bi}^{2} \mathrm{O}^{3}-\mathrm{B}^{2} \mathrm{O}^{3}$, E. M. Levin and C. L. McDaniel, J. Äm. Ceram. Soc. 45, No. 8, 355-360 (Aug. 1962).

Electron energy losses in solids and their influence on the electron diffraction diagram, L. Marton, J. Phys. Soc. Japan 17, Suppl. B-II, 68-73 (1961).
Scattered radiation from large Cs 137 sources, L. Costrell, Health Phys. 8, No. 5, 491-498 (Oct. 1962).

Optical detection of microwave transitions in electronically excited CN produced by a chemical reaction, R. L. Barger, H. P. Broida, A. J. Estin, and H. E. Radford, Phys. Rev. Letters 9, No. 8, 345-346 (Oct. 15, 1962).

Sum rules for vibrational-rotational energy levels including centrifugal distortion, H. C. Allen, Jr. and W. B. Olson, J. Chem. Phys. 37, No. 2, 212-214 (July 15, 1962).

Optical constants of aluminum in vacuum ultraviolet, $R$. LaVilla and H. Mendlowitz, Phys. Rev. Letters 9, No. 4, 149-150 (Aug. 15, 1962).

Microwave spectrum of methyldifluoroarsine, L. J. Nugent and C. D. Cornwell, J. Chem. Phys. 37, No. 3, 523-534 (Aug. 1962).

Standard tests for electrical properties, A. H. Scott, SPE J. 18, 1375-1378 (Nov. 1962).

The industrial significance of the National Bureau of Standards research, G. M. Kline, Mod. Plastics 40, No. 2, 149 (Oct. 1962)

Comments on "A new precision low level bolometer bridge," G. F. Engen, Proc. IRE 50, No. 9, 1997 (Sept. 1962).

Solar particles in interplanetary space, C. S. Warwick, Sky and Telescope XXIX, No. 3, 133-136 (Sept. 1962).

The kinectics of the heat preciptation of collagen, J. M. Cassel, L. Mandelkern, and D. E. Roberts, J. Am. Leather Chem. Assoc. LVII, No. 11, 556-575 (Nov. 1962).

Chromium plating by thermal decomposition of dicumene chromium, W. H. Metzger, Jr. Plating 49, No. 11, 1176 (Nov. 1962).

Oxidation of high polymers, M. Tryon and L. A. Wall, Book, Autoxidation and Antioxidants II, Chapt. 19, p. 919 (Interscience Publ. Inc., New York, N.Y., 1962).

New wavemeter for millimeter wavelengths, R. W. Zimmerer, Rev. Sci. Instr. 33, No. 8, 858-859 (Aug. 1962).

Vibrational-rotational spectroscopy, H. C. Allen, Jr., and W. B. Olson, Ann. Rev. Phys. Chem. 13, 221-240 (1962).

The use of a Venturi tube as a quality meter, R. V. Smith, P. C. Wergin, J. F. Ferguson, and R. B. Jacobs, J. Basic Eng. 84, 411-412 (Sept. 1962).

Dislocation loops in deformed copper, A. W. Ruff, Jr., Fifth Intern. Congress for electron Microscopy, p. J-10 (Academic Press, Inc., New York, N.Y., 1962).

Applications of resistance thermometers to calorimetry, G. T. Furukawa, Book, Temperature, Its Measurement and Control in Science and Industry 3, Pt. 2, 317-328 (1962).

Absorption spectrum of carbon vapor in solid argon at $4^{\circ}$ and $20^{\circ}$ K, R. L. Barger and H. P. Broida, J. Chem. Phys. 37, No. 5, 1152-1153 (Sept. 1, 1962).

Rotation-vibration spectrum of matrix-isolated hydrogen chloride, L. J. Schoen and D. E. Mann, J. Chem. Phys. 37, No. 5, 1146-1147 (Sept. 1, 1962)

Very-low-frequency phase observations on the ionospheric effects of the solar flare of September 28, 1961, A. G. Jean and J. H. Crary, J. Geophys. Res. 67, No. 12, 4903-4905 (Nov. 1962).

The radiant energy from sources in the far infrared, E. K. Plyler, D. Yates, and H. A. Gebbie, J. Opt. Soc. Am. 52, No. 8, 859-861 (Aug. 1962).

Obtaining the internal junction characteristics of a transistor for use in analog simulation, S. B. Geller, IRE Trans. Electron. Computers EC-11, No. 5, 709-710 (Oct. 1962).

Graphical evaluation of analytical results, W. J. Youden, Proc. Conf. Chemical Control Problems, p. 1-15 (Spon. Natl. Plant Food Inst., Washington, D.C., 1959).

The measurement of ionospheric drifts by means of a Doppler technique, K. Davies, J. Geophys. Res. 67, No. 12, 49094913 (Nov. 1962)

Laboratory astrophysics, L. M. Branscomb and R. N. Thomas, Phys. Today 15, 42-44, 46 (Nov. 1962).

Standards - The key to success in engineering, A. T. MePherson, Rev. Centro Estud. Ing. 130, No. 656, 352-355 (1961).

The thermodynamic scale of temperature below $1^{\circ} \mathrm{K}, \mathrm{R}$. P. Hudson, Book, Temperature Its Measurement and Control In Science and Industry 3, Pt. I, 51-57 (Reinhold Publ. Co., New York, N.Y., 1962).

Precision phase meter, D. M. Waters, D. Smith, and M. C. Thompson, Jr., IRE Trans. Instr. I-II, 64-66 (Sept. 1962). 
Nuclear photoeffect in deformed nuclei, E. Hayward, Rendiconti Della Scuola Intern. Fisica "Enrico Fermi" XV, 214-222 (Academic Press Inc., New York, N.Y., 1962).

The effect of temperature and humidity on the oxidation of air-blown asphalts, P. G. Campbell, J. R. Wright, and P. B. Bowman, Mater. Res. Std. 2, No. 12, 988-995'(Dec. 1962).

Standards for the future, R. D. Huntoon, Proc. Annual Spring Conf. Standards Engineers Society, Boston Section and Company Member Conf. of American Standards Association, May 24-25, 1962 (Boston, Mass., 1962).

Intramolecular rearrangements in the solid phase photolysis of 4-methyl-2-hexanone and sec-butyl acetate, R. E. Rebbert and P. Ausloos, J. Chem. Phys. 37, No. 5, 1158-1159 (Sept. 1, 1962).

The vapor phase fluorescence and its relationship to the photolysis of propionaldehyde and the butyraldehydes, R. P. Borkowski and P. Ausloos, J. Am. Chem. Soc. 84, No. 21, 4044-4048 (Nov. 5, 1962).

Relaxation of nonequilibrium distributions, K. E. Shuler, Book, Temperature, Its Measurements and Control in Science and Industry 3, Pt. I, 27-34 (Reinhold Publ. Co., New York, N.Y., 1962).

Crosslinking of polymers induced by excited species, L. A. Wall and R. B. Ingalls, J. Polymer Sci. Letter to Editor 62, No. 173, S5-S6 (1962).

Standard electromotive force of the cell $\mathrm{H}_{2} ; \mathrm{HBr}(\mathrm{m}) ; \mathrm{AgBr}$; $\mathrm{Ag}$ from 0 to $50^{\circ}$, H. B. Hetzer, R. A. Robinson, and R. G. Bates, J. Phys. Chem. 66, No. 8, 1423-1426 (1962).

Vacuum ultraviolet photochemistry. IV. Photolysis of propane, H. Okabe and J. R. McNesby, J. Chem. Phys. 3\%, No. 6, 1340-1346 (Sept. 1962).

The thermodynamic temperature scale, Its definition and realization, C. M. Herzfeld, Book, Temperature, Its Measurement and Control in Science and Industry 3, Pt. I, 41-50 (Reinhold Publ. Co., New York, N.Y., 1962).

Interpretation of rate experiments with resolved quantum levels, T. Carrington, Discussions Faraday Soc., No. 33, 44-51 (1962).

Hyperfine structure and intercombination line intensities in the spectra of magnesium, zinc, cadmium, and mercury, R. H. Garstang, J. Opt. Soc. Am., 52, No. 8, 845-851 (Aug. 1962).

Conformation and frictional properties of polystyrene in dilute solutions, D. MeIntyre, A. Wims, L. C. Williams, and L. Mandelkern, J. Phy. Chem. 66, 1932-1940 (Oct. 1962).

Use of a "peek-a-boo" information retrieval technique for a personal reference file, J. A. Bennett, J. Wash. Acad. Sci. 52, No. 9, 216-219 (Dec. 1962).

Photographic strain measuring technique for use above 3,000 F, L. Mordfin and T. Rubusto, Jr., Prov. Instr. Soc. Am. 17, Pt. 1, 3.4.62-1 (1962).

Complex formation of monomeric amides with lithium perchlorate, A. F. Diorio, E. Lippincott, and L. Mandelkern, Nature 194, No. 4848, 1296-1297 (Sept. 1962).

Absorption bands of carbon dioxide from 2.8 to $4.2 \mu$, E. K. Plyler, E. D. Tidwell, and W. S. Benedict, J. Opt. Soc. Am. 52, No. 9, 1017-1022 (Sept. 1962).

The speed of processes involved in electroplating: movement of solute, attainment of the steady state and formation of metal, A. Brenner, 49th Annual Tech. Proc. Am. Electroplaters Soc. p. 9-13 (1962).

Synthesis of higher ketoses by aldol reactions. I. Three D-heptuloses, R. Schaffer and H. S. Isbell, Org. Chem. 27, 3268-3270 (Mar. 1962).

Growth rates of potassium crystal from the vapor phase, R. L. Parker, J. Chem. Phys. 37, No. 8, 1600-1605 (Oct. 1962).

Ranking laboratories by round-robin tests, W. J. Youden, Mater. Res. Std. 3, No. 1, 9-13 (Jan. 1963).

The NBS photoelectric pyrometer of 1961, R. D. Lee, Book, Temperature, Its Measurement and Control in Science and Industry 3, Pt. 1, 507-515 (Reinhold Publ. Corp., New York, N.Y., 1962).

The error rate in a multiple-frequency-shift system and the output signal/noise ratio in a frequency modulation and a pulse-code-modulation/frequency-shift system, H. Akima,
Intern. Conf. Satellite Communication, p. 305-310 (Nov. $22-28,1962)$.

Heat transfer in intensively outgassed powders, R. C. Little, F. G. Carpenter, and V. R. Deitz, J. Chem. Phys. 37, No. 8, 1896-1898 (Oct. 15, 1962).

Rates of thermal degradation of organic polymers, S. L. Madorsky, SPE J. 18, No. 12, 1482-1490 (Dec. 1962).

Vacuum ultraviolet photochemistry. V. Photolysis of isobutane, H. Okabe and D. A. Becker, J. Am. Chem. Soc. 84, No. 21, 4004-4007 (1962).

Traces of products of angular momentum matrices. II. Spherical basis, E. Ambler, J. C. Eisenstein, and J. F. Schooley, J. Math. Phys. 3, No. 4, 760-771 (July-Aug. 1962).

A study of stability of high temperature platinum resistance thermometers, J. P. Evans and G. W. Burns, Book, Temperature, Its Measurement and Control in Science and Industry 3, Pt. 1, 313-318 (Reinhold Publ. Corp., New York N.Y., 1962).

The present state of atomic spectra, C. M. Moore-Sitterly, Japan Anal. 11, No. 11, 1199-1202 (1962).

Correlation factors for impurity diffusion - fcc lattice, J. R. Manning, Phys. Rev. 128, No. 5, 2169-2174 (Dec. 1962).

A differential thermocouple voltmeter, J. E. Griffin and F. L. Hermach, AIEE Trans., Pt. I. Communications and Electronics No. 63, Article No. 62-819, 338-344 (Nov. 1962).

Color tests for antioxidants, E. J. Parks, L. T. Milliken, and F. J. Linnig, Rubber Age 92, No. 2, 257-261 (Nov. 1962).

Cavitation problems in cyrogenics, R. B. Jacobs and K. B. Martin, J. Basic Eng. 82, 756-757 (Sept. 1960).

Experimental proof of the absence of equilibrium in a helium arc, A. T. Hattenburg and H. J. Kostkowski, Book, Temperature, Its Measurement and Control in Science and Industry 3, Pt. 1, 587-592 (Reinhold Publ. Corp., New York, N.Y., 1962).

Technical training in the weights and measures program, M. W. Jensen, Scale J. 49, No. 4, 4-5 (Jan. 1963) ; Southern Weights and Measures Program 14, No. 12, 2-5 (Dec. 1962).

Corrosion rates of ferrous alloys (Fe, Cr, and $\mathrm{Fe}-\mathrm{Cr}-\mathrm{Si}$ ) measured by polarization techniques, W. J. Schwerdtfeger, Corrosion 19, No. 1, 17t-25t (Jan. 1963).

Survey of U.S.A. ionospheric research 1957-1959, T. N. Gautier, (Triennium Report of U.S. Natl. Comm. IUGG), Trans. Am. Geophys. Union 41, No. 2, 232-236 (June 1960).

On the analysis of polarization rotation recordings of satellite radio signals, R. S. Lawrence and C. G. Little, Intern. Sci. Radio Union, IGY Comm. Some Ionospheric Results Obtained During the IGY; Proc. Symp. Organized by URSI/ AGI Comm. Brussels, Belgium, p. 391-399 (Elsevier Publ. Co., Amsterdam, The Netherlands, 1960).

Widely separated clocks with microsecond synchronization and independent distribution system, T. L. Davis and R. H. Doherty, IRE WESCON Conv. Pt. 5, p. 3-17 (1960).

Dissociation constant of $t$-butylammonium iom and related thermodynamic quantities from 5 to $35^{\circ}$, H. B. Hetzer, R. A. Robinson, and R. G. Bates, J. Phys. Chem. 66, 2696 (1962)

Memorial to Sir Ronald Aylmer Fisher: 1890-1962, W. J. Youden, J. Am. Stat. Assoc. 5\%, No. 300, 727-728 (Dec. 1962).

Measurement of electron density and temperature in dense plasma by application of line broadening theory, J. B. Schumaker, Jr., and W. L. Wiese, Book, Temperature, Its Measurement and Control in Science and Industry 3, Pt. 1, 575-579 (Reinhold Publ. Corp., New York, N.Y., 1962).

Vapor phase growth kinetics of $\mathrm{Hg}$ and $\mathrm{K}$ whiskers by field emission, R. L. Parker and S. C. Hardy, J. Chem. Phys. 37, No. 8, 1606-1609 (Oet. 1962).

A low impedance Maxwell bridge for measuring toroidallyshaped magnetic materials from 1 Ke to $100 \mathrm{Kc}, \mathrm{A}$. L. Rasmussen and R. C. Powell, Proc. IRE 50, 2505-2506 (Dec. 1962).

Germanium vacuum ultraviolet Ritz standards, V. Kaufman and K. L. Andrew, J. Opt. Soc. Am. 52, No. 11, 1223-1237 (Nov. 1962).

Radio properties of aurorae, C. G. Little, U.S.A. Natl. Comm. Intern. Sci. Radio Union Report to Natl. Acad. Sci.-Natl. Res. Council on 13th Gen. Assembly, Sept. 5-15, 1960, 
London, England, p. 216-270 (Natl. Acad. Sci--Natl. Res. Council, Wash., D.C., 1961).

The determination of absolute temperatures from sound velocity measurements, G. Cataland, M. H. Edlow, and H. H. Plumb, Book, Temperature, Its Measurement and Control in Science and Industry 3, Pt. 1, 129-132 (Reinhold Publ. Corp., New York, N.Y., 1962).

The role of temperature in our measuring system, A. G. McNish, Book, Temperature, Its Measurement and Control in Science and Industry 3, Pt. 1, 35-38 (Reinhold Publ. Corp., New York, N.Y., 1962).

Electrophoretic deposits of barium titanate, V. A. Lamb and H. I. Salmon, Am. Ceram. Soc. 41, No. 11, 781-782 (Nov. 1962).

A rapid method of estimating the order of chemical reactions, J. H. Flynn, Chem. Eng. 69, 137-140 (Aug. 1962).

Microwave Doppler measurements of the ionization front in cylindrical shock waves from exploding wires, D. L. Jones and R. M. Gallet, Exploding Wires 2, 127-144 (1962).

Temperature dependence of elastic constants of vitreous silica, S. Spinner, J. Am. Ceram. Soc. 45, No. 8, 394 (Aug. 1962).

Comments on the use of net rate processes and the equivalent two-level atom in non-LTE computations, R. N. Thomas, Ann. d'Astrophys. 23, No. 6, 871-878 (1961).

Comments on the limits to the refractive index at ground level as a radio-meteorological parameter, B. R. Bean and G. D. Thayer, Proc. IRE 48, No. 8, 1498-1501 (Aug. 1960).

Rate of spherulitic crystallization with chain folds in polychlorotrifluoroethylene, J. D. Hoffman and J. J. Weeks, J. Chem. Phys. 3\%, No. 8, 1723-1741 (Oct. 1962).

Energy exchange in the biosphere, D. M. Gates, Book (Harper and Row Publ., New York, N.Y., 1962).

On the propagation of ELF pulses in the earth-ionosphere waveguide, J. R. Wait, Can. J. Phys. 40, 1360-1369 (Oct. 1962).

An experimental investigation of over-all heat transfer coefficients for condensing and boiling hydrogen films, $\mathrm{K}$. D. Timmerhaus, D. E. Drayer, and J. W. Dean, Intern. Develop. Heat Transfer (ASME) 2, 270-278 (1961).

The photolysis and radiolysis of $\mathrm{CH}_{3} \mathrm{~N}_{2} \mathrm{CH}_{3}$ and $\mathrm{CH}_{3} \mathrm{~N}_{2} \mathrm{CH}_{3}-$ $\mathrm{CD}_{3} \mathrm{~N}_{2} \mathrm{CD}_{3}$ mixtures, R. E. Rebbert and P. Ausloos, J. Phys. Chem. 66, No. 11, 2253-2258 (Nov. 1962).

Isotope effect in the recrystallization of D-mannose-1- $t$ phenylhydrazone, F. Weygand, H. Simon, K. D. Keil, H. S. Isbell, and L. T. Sniegoski, Anal. Chem. 34, 1753-1755 (Dec. 1962).

Interpretation of $p \mathrm{H}$ measurements in alcohol-water solvents, R. G. Bates and R. A. Robinson, Proc. 7th Intern. Conf. Coordination Chemistry, Stockholm, Sweden, p. 342-344 (1962).

Polymorphism in fibrous polypeptides: $\alpha \rightleftarrows \beta$ transformation in naturally occurring keratin, A. F. Diorio, L. Mandelkern, and E. R. Lippincott, J. Phys. Chem. 66, No. 11, 20962100 (Nov. 1962)

Recent radar observations of new forms of ionosphere scatter, K. L. Bowles, U.S.A. Natl. Comm. Intern. Sci. Radio Union Report to Natl. Acad. Sci.-Natl. Res. Council on 13th Gen. Assembly, Sept. 5-15, 1960, London, England, p. 288-295 (Natl. Acad. Sci.-Natl. Res. Council, Wash. D.C., 1961).

The significance of transients and steady-state behavior in nonlinear systems, W. J. Hartman, Proc. IRE Correspondence Section 49, No. 3, 637 (Mar. 1961).

Reaction of methyl- $d_{2}$ radicals with isobutane, isobutane-2- $d$ and propane, W. M. Jackson, J. R. McNesby, and B. deB. Darwent, J. Chem. Phys. 37, No. 8, 1610-1615 (Oct. 1962).

Photoionization from outer atomic subshells, A model study, J. W. Cooper, Phys. Rev. 128, No. 2, 681-693 (Oct. 15, 1962).
Solar flare effects in the $F$-region of the ionosphere, R. W. Knecht and R. E. McDuffie (Proc. Intern. Conf. Cosmic Rays and the Earth Storm), J. Phys. Soc. Japan 17, Suppl. A, Pt. I, 280-285 (1962).

Theory and methods of optical pyrometry, H. J. Kostkowski and R. D. Lee, Book, Temperature, Its Measurement and Control in Science and Industry 3, Pt. 1, 449-481 (Reinhold Publ. Corp., New York, N.Y., 1962).

Recent experiments on liquid helium vapor pressure measurements from $2^{\circ}$ to $4^{\circ} \mathrm{K}, \mathrm{G}$. Cataland, M. H. Edlow, and H. H. Plumb, Book, Temperature, Its Measurement and Control in Science and Industry 3, Pt. 1, 413-417 (Reinhold Publ. Corp., New York, N.Y., 1962).

Measurement of effective temperatures of microwave noise sources, J. S. Wells, W. C. Daywitt, and C. K. S. Miller, IRE Intern. Conv. Record, Pt. 3, 220-238 (1962).

Low-level low-frequency detection system, N. T. Larsen Rev. Sci. Instr. 33, 1200-1208 (Nov. 1962)

The calibration of temperature standards on the international practical temperature scale of 1948 , J. P. Evans, Proc. 17th Ann. Instrument-Automation Conf. Exhibit, Instr. Soc. Am. Preprint No. 21-1-62 (New York, N.Y., Oct. 1962).

On the extension of the low-chromosphere model to the region of origin of the UV solar spectrum, R. N. Thomas (Proc. 10th Astrophysical Symp. Liege, Belgium), Mem. Roy. Soc. Belgium 20, 305 (1961).

The text revision of the international temperature scale of 1948, H. F. Stimson, Book, Temperature, Its Measurement and Control in Science and Industry 3, Pt. 1, 59-66 (Reinhold Publ. Corp., New York, N.Y., 1962).

Analysis of rotation errors of a waveguide theory vane antennuator, W. Larson, IRE Intern. Conv. Record, Pt. 3, 213-219 (1962)

An improved resistance thermometer bridge, J. P. Evans, Book, Temperature, Its Measurement and Control in Science and Industry 3, Pt. 1, 285-289 (Reinhold Publ. Corp., New York, N.Y., 1962).

The structure of lithium dipotassium trimetaphosphate monohydrate, E. D. Eanes and H. M. Ondik, Acta Cryst. 15, Pt. 12, 1280-1285 (Dec. 1962).

Resistance thermometry in the liquid helium temperature region, M. H. Edlow and H. H. Plumb, Book, Temperature, Its Measurement and Control in Science and Industry 3, Pt. 1, 407-411 (Reinhold Publ. Corp., New York, N.Y., 1962).

Airglow research in the United States, F. E. Roach, (Triennium Report of U.S. Natl. Comm. IUGG) Trans. Am. Geophys. Union 41, No. 2, 242-243 (1960).

A National Bureau of Standards gas thermometer, L. A Guildner, Book, Temperature, Its Measurement and Control in Science and Industry 3, Pt. 1, 151-155 (Reinhold Publ. Corp., New York, N.Y., 1962).

Quartz crystals at low temperatures, P. R. Simpson and A. H. Morgan, Proc. 13th Annual Symp. Frequency Control, p. 207-231 (Aug. 1959).

Precursor electrons ahead of cylindrical shock waves, D. L. Jones, Phys. Fluids 5, No. 9, 1121-1122 (Sept. 1962).

The thermal conductivity of solid nitrogen, H. M. Roder, Letter to Editor Cryogenics \%, No. 5, 302-304 (Sept. 1962). High pressure transition in RbF, G. Piermarini and C. E. Weir, J. Chem. Phys. 37, No. 8, 1887-1888 (Oct. 15, 1962).

* Publications for which a price is indicated are available from the Superintendent of Documents, U.S. Government Printing Office, Washington 25, D.C. (foreian postage onefourth additional). Reprints from outside journals and the NBS Journal of Research may often be obtained directly from the authors. 